\title{
Two medium size cavitation tunnel hydro-acoustic benchmark experiment comparisons as part of a round robin test campaign
}

\author{
Giorgio Tani', Batuhan Aktas ${ }^{2, *}$, Michele Viviani ${ }^{1}$, Mehmet Atlar \\ ${ }^{1}$ Department of Electrical, Electronic, Telecommunication Engineering and Naval \\ Architecture, (DITEN), University of Genova, Genova, Italy \\ ${ }^{2}$ Naval Architecture, Ocean \& Marine Engineering, University of Strathclyde \\ Glasgow, UK \\ Corresponding Author: \\ Batuhan Aktas, batuhan.aktas@strath.ac.uk \\ Henry Dyer Building \\ 100 Montrose Street \\ Naval Architecture, Ocean \& Marine Engineering, \\ University of Strathclyde \\ Glasgow G4 0LZ, UK \\ Tel: +44 (0) 1415484094
}




\begin{abstract}
:
The increase in marine transportation in the last decades has resulted in a rise of the different emissions linked to it. Amongst various detrimental emissions of shipping activities, underwater noise is known to affect ambient noise levels and hence threaten the life (or even survivability) of marine mammals. This concern brought the underwater radiated noise (URN) to the attention of regulators, considering the possible need to limits for commercial ships, thus resulting in a surge of interest.
\end{abstract}

As being the main contributor to the URN of ships, the accurate prediction of propeller cavitation and hence associated noise in the design stage is crucial for achieving reductions in terms of emitted sound pressure levels. Whilst computational methods are developing at an exponential pace and so are prospective tools for the future, model scale experiments still represent the most reliable and largely adopted approach for the prediction of propeller radiated noise. Despite the importance of model tests, being the only reliable tool for cavitation noise prediction, a benchmark data is non-existent for facilities all over the world to compare and correlate their noise measurements.

Within this framework, this paper presents the first comparison of a round robin test campaign amongst the Noise CoP (Community of Practice) of Hydro Testing Forum (HTF). Based on the extensive experiments conducted first at Emerson Cavitation Tunnel (ECT), a reduced test matrix is proposed to the forum members. University of Genoa (UNIGE) is the first member to complete the tests specified in the reduced matrix and this paper presents comparisons between the test results of ECT and UNIGE cavitation tunnel in terms of measured sound pressure levels, propeller open water performance, cavitation observations and cavitation inception characteristics. Moreover, in order to shed a light on the issue of propeller cavitation noise measurements, a series of investigations are carried out by UNIGE scrutinizing the effect of hydrophone position, oxygen content, propeller shaft revolution rate, sensitivity to thrust coefficient and cavitation number.

Keywords: Model tests, Cavitation, Underwater radiated noise, Inclined shaft effect, Systematic propeller tests, Round robin noise tests 


\section{Nomenclature:}

\begin{tabular}{|c|c|c|c|}
\hline$\mu$ & Dynamic fluid viscosity [ $\mathrm{kg} / \mathrm{ms}]$ & $P_{\text {atm }}$ & Atmospheric pressure $[\mathrm{Pa}]$ \\
\hline$A_{E}$ & Expanded blade area $\left[\mathrm{m}^{2}\right]$ & $P_{v}$ & Vapour Pressure [Pa] \\
\hline$A_{E} / A o$ & Expanded Blade Area Ratio & $Q$ & Torque $[\mathrm{Nm}]$ \\
\hline$A_{\circ}$ & Propeller disc area [m2] & $R$ & Radius $[m]$ \\
\hline$C_{0.7 R}$ & 0.7 Radius chord length $[\mathrm{m}]$ & $r$ & $\begin{array}{l}\text { Distance between the acoustic center of the noise source } \\
\text { and hydrophone }[\mathrm{m}]\end{array}$ \\
\hline$D$ & Diameter $[\mathrm{m}]$ & $\rho$ & Fluid density [kg/m3] \\
\hline$f$ & Frequency [Hz] & $R e$ & Reynolds number \\
\hline$g$ & Gravitational acceleration $\left[\mathrm{m} / \mathrm{s}^{2}\right]$ & $r_{r e f}$ & Reference distance (1m) \\
\hline$h$ & Shaft immersion $[\mathrm{m}]$ & $S P L$ & Sound pressure level $[d B]$ \\
\hline$J$ & Advance coefficient & $T$ & Thrust [N] \\
\hline$K_{P}$ & Pressure coefficient & $V$ & Speed $[\mathrm{m} / \mathrm{s}]$ \\
\hline$K_{Q}$ & Torque coefficient & $v$ & Kinematic fluid viscosity $\left[\mathrm{m}^{2} / \mathrm{s}\right]$ \\
\hline$K_{T}$ & Thrust coefficient & $V_{A}$ & Advance speed $[\mathrm{m} / \mathrm{s}]$ \\
\hline$L_{K P}$ & Pressure coefficient level $[d B]$ & $Z$ & Propeller blade Number \\
\hline$N$ & Rotational speed [RPM] & $\eta_{o}$ & Propeller efficiency \\
\hline$n$ & Propeller revolution speed [rps] & $\sigma_{N}$ & Rotational cavitation cumber \\
\hline$P$ & Pressure $[\mathrm{Pa}]$ & $\sigma_{V}$ & Free stream cavitation number \\
\hline$P / D$ & Pitch ratio at $0.7 R$ & & \\
\hline
\end{tabular}

\section{Introduction}

Marine traffic and general shipping activities have continuously increased during the $20^{\text {th }}$ century and are still enlarging (Hildebrand, 2009). During last decades, the problem of unavoidable impact of these activities has become a great concern and hence large efforts have been spent to limit this impact, by improving the ship performances from the point of view of general emissions.

As one of these emissions, underwater ship radiated noise (URN) and its effects on marine life have been under the spotlight. In fact, noise levels in the seas have increased, especially near harbours, and this may affect the normal life of the marine fauna. As part of their daily survival 
marine mammals heavily rely on noise for swimming, communicating and looking for food (Richardson et al., 2013; White and Pace, 2010). Due to this fact the URN control is no more limited to naval vessels which has been the case until recently since World War II. The significant number of research projects carried out in recent years at both national and international levels reflects this increasing attention on the problem of shipping noise.

As an example, the European Union promoted different international research projects, among which the SILENV (Ships oriented Innovative soLutions to rEduce Noise and Vibrations) project was directly centred on the problem of ship noise. Other projects, such as BESST (Breakthrough in European Ship and Shipbuilding Technologies), despite not being entirely dedicated to the problem of radiated noise, presented considerable activities dedicated to that field. In the last years, two further projects, i.e. AQUO (Achieve QUieter Oceans) and SONIC (Suppression Of underwater Noise Induced by Cavitation) (SONIC, 2012), were directly focussed on the problem of cavitation related noise. Moreover, the EU has established the Marine Strategy Framework Directive (MSFD) to investigate and implement programmes of measures which are designed to achieve or maintain 'Good Environmental Status' in the marine environment (Van der Graaf et al., 2012). In complementing the above activities Joint Research Programme (JRP)10, which was called "Noise Measurements", was established within the Hydro Testing Alliance-Network of Excellence (HTA-NoE) and successfully completed its mission in 2011 (AMT 2011). The members of JRP10 decided to form a working group to investigate URN issues further. This working group, which was initially named "Noise Working Group" (NWG), later has become "Noise Community of Practice (Noise CoP)" of Hydro Testing Forum (HTF) as a longer continuation of the HTA-NoE, which was phased out by then (AMT 2013).

The acoustic characterisation of ships may be a very difficult task, due to the overall complexity of the object under studies and to the simultaneous presence of different noise generation mechanisms, which sometimes may also interact together (Ross, 1976). One of the main sources of URN by ships is the propeller, especially when cavitating (Abrahamsen, 2012; Arveson and Vendittis, 2000). Consequently, great attention is devoted to the acoustical characterization of propeller cavitation (Renilson, 2009). 
Nowadays the study of propeller performances and cavitation is performed through both model scale experiments and numerical simulations. Actually, the capabilities of numerical tools are continuously improving, thanks to the increasing computational power, and higher order methods are utilized for simulation of complicated phenomenon (Bensow \& Bark, 2010). Such complex simulations are able to simulate complex turbulent flows, cavitation and even noise. However, the latter is still a difficult task because of the extreme computational cost of such simulations and of the difficulties in accurate modelling of the broadband components of the pressure fluctuations. As a result, model scale experiments still represent the most reliable and largely adopted approach for the prediction of propeller radiated noise (Bertschneider et al., 2014).

Despite the above described fact, several aspects of model scale noise testing are still not clear, as various scale effects on cavitation and noise or the effects of the characteristics of the facilities where tests are conducted. Due to this, research institutes performing cavitation and noise tests are always interested in the development of suitable testing procedure, to the enhancement of the measuring facilities or to the study of scaling laws to be applied for the prediction of full-scale noise spectra. From this point of view, international collaborations are of great importance in order to share knowledge and to uniform as much as possible techniques and procedures.

This is, as an example, one of the main aims of the ITTC (International Towing Tank Conference) and that of organizations like the Hydro-Testing Alliance Network of Excellence (HTA-NoE) and Hydro-Testing Forum (HTF). ECT of Newcastle University and UNIGE are both participants of HTF. In particular, they are involved, together with a large number of research institutes, in a working group devoted to the study of propeller radiated noise, the Noise Community of Practice (Noise CoP). The first important goal of this group has been to perform a round robin test campaign for the noise characterization of the propeller of the University of Newcastle catamaran "The Princess Royal”. 
In this campaign, tests are being carried out in facilities with very different characteristics, such as cavitation tunnels of different sizes, the Marin Depressurized Towing Tank (Bosschers et al., 2013) and the INSEAN circulating channel (Costanzo and Elefante, 1999). The comparison of results obtained with such a variety of structures and their own respective procedures constitutes one of the more attractive points of the whole round robin campaign.

The model propeller used in the round robin tests campaign has been the object of extensive tests at the Emerson Cavitation tunnel, as the $1^{\text {st }}$ tests in the campaign, considering a large set of operational conditions and different configurations in uniform inflow with different shaft inclinations (Aktas et al., 2015) and the real ship wake field reproduced in the tunnel by means of a dummy model plus wire screens, as part of the SONIC project activities (Aktas et al., 2016).

For the round robin it was decided to limit the complexity of the configuration, thus the ship nominal wake field was disregarded, preferring the uniform inflow with the inclined shaft to create an oblique flow condition.

In this paper, the first results of the campaign with the reduced test matrix are presented in comparative manner by using the test results carried out at the Emerson Cavitation Tunnel (ECT) and those obtained at the University of Genova (UNIGE) cavitation tunnel, which is the second institution, after Newcastle University, completing its series of tests in the round robin campaign. The comparisons include not only radiated noise, but also inception tests and cavitation extents. This presents further invaluable data for this interesting case study to discuss possible issues and discrepancies in relation with the different characteristics and testing procedures of the two facilities. 


\section{Experimental Setup}

\subsection{The Emerson Cavitation Tunnel}

The tests were performed at Emerson Cavitation Tunnel (ECT) of the School of Marine Science and Technology at Newcastle University. This tunnel is a vertical plane, closed circulating design and capable to reach flow speeds up to $15.5 \mathrm{knots}(8 \mathrm{~m} / \mathrm{s})$. The size range of the model scale propellers that can be tested, vary from $150 \mathrm{~mm}$ to $400 \mathrm{~mm}$ depending on the type of test. The round robin test campaign was performed using Kempf \& Remmers H33 propeller dynamometer to power the propeller. A schematic representation of the ECT is provided by Figure 1.

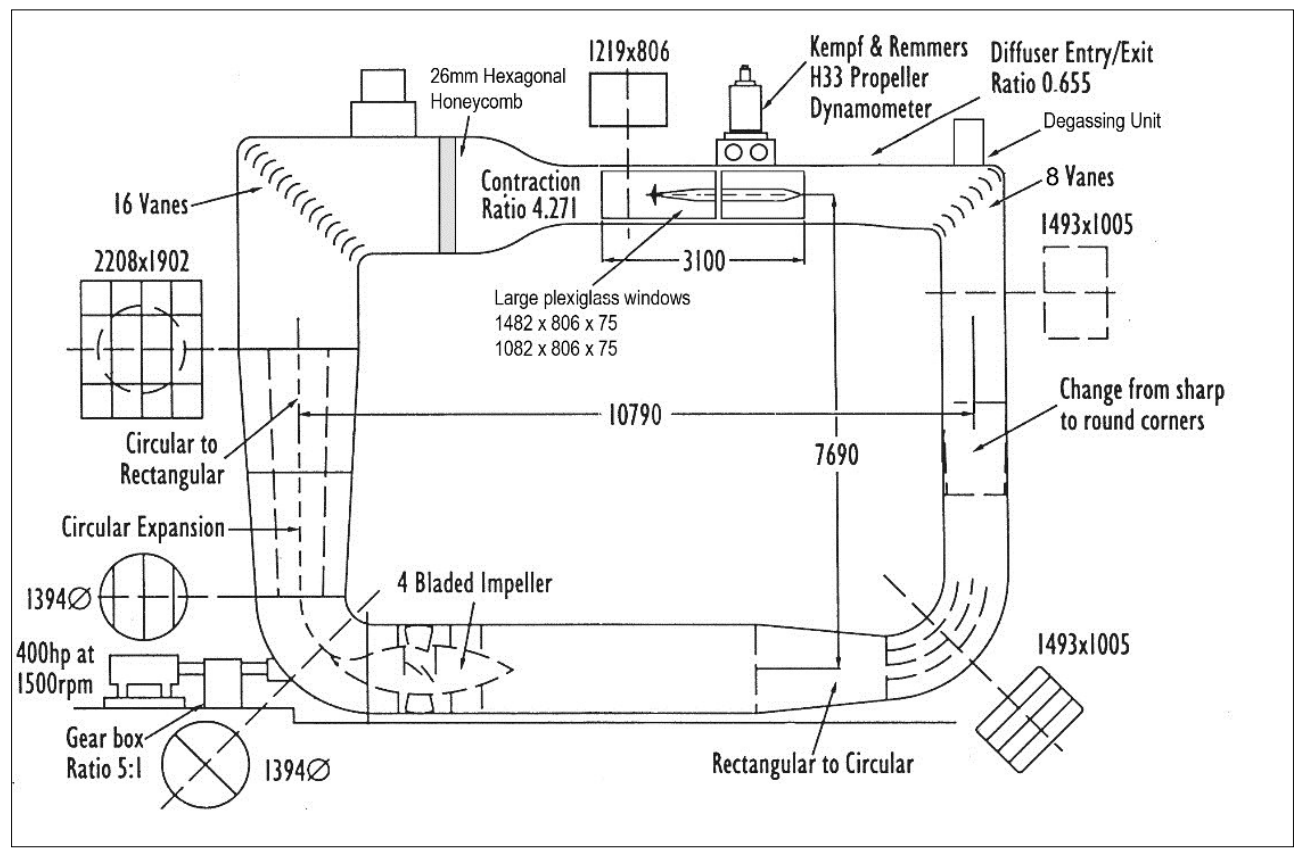

Figure 1 Emerson Cavitation Tunnel (Atlar, 2011)

The Kempf \& Remmers H33 dynamometer, with its torpedo-shaped outline, is commonly used for open water experiments due to its relatively larger range of thrust and torque compared to the Kempf \& Remmers R45. The dynamometer is attached to the driving motor by means of a Cussons type H101-27 system for shaft height and angle adjustment, which facilitates testing for propeller inclination as well as yawing, pitching and heaving effects. The H33 is able to measure torque values up to $147 \mathrm{Nm}$ and thrust values up to $2943 \mathrm{~N}$ with maximum permissible revolutions of 4000 RPM. Further details of the Emerson Cavitation Tunnel and equipment can be found in (Atlar, 2011) 
The water quality and temperature of the ECT is monitored throughout all cavitation tests as it has a significant impact on the measurements. The water temperature of the tunnel water varied between $23^{\circ} \mathrm{C}$ to $25^{\circ} \mathrm{C}$ during the course of the experiments. The tunnel water is thus brought to $30 \%$ total gas content as suggested by the ITTC $(2011,1987)$. ECT has a dedicated water quality monitoring system and degassing system to keep the oxygen saturation level of the tunnel at desired level. The tunnel water quality is recorded using two systems in conjunction: the first device, which is MS5 mini sonde and its dedicated Hydrolab software, enables continuous monitoring of the water quality (OTT, 2006). The probes attached to the tip of MS5 measure the water quality within a tubing arrangement through which tunnel water is discharged using a peristaltic pump; the second device is a handheld meter for dissolved oxygen, from YSI instruments (YSI Environmental, 2006). For the current study, measurements are made using the latter.

The most commonly used observation technique for the cavitation tunnel tests are the highspeed camera recordings aided with stroboscopic lighting which is synchronized with shaft position signal. This enables freezing the image due to the triggered flash provided by the strobe lights. Stroboscope lighting also accommodates the still imaging with Nikon D90 Digital Single-Lens Reflex (DSLR) camera by reducing the shutter speed in order to fit the image acquisition into the short bursts of the strobe flash.

Noise measurements were recorded using a Bruel and Kjaer type 8103 miniature hydrophone mounted in a water filled, thick walled steel cylinder placed on the outside of the tunnel's plexiglas window. This cylinder was glued onto the starboard window level with the center of the propeller boss when the shaft inclination is zero and at a horizontal distance of $0.61 \mathrm{~m}$ from the shaft centreline as presented by Figure 2. The noise data acquisition for 1/3 Octave band was conducted using the multi-buffer option of the Bruel\&Kjaer PULSE software which triggers the system every 0.25 seconds for the next measurement. Each measurement were recorded for 200 triggers or 50 seconds at $45 \mathrm{kHz}$ sampling rate. 


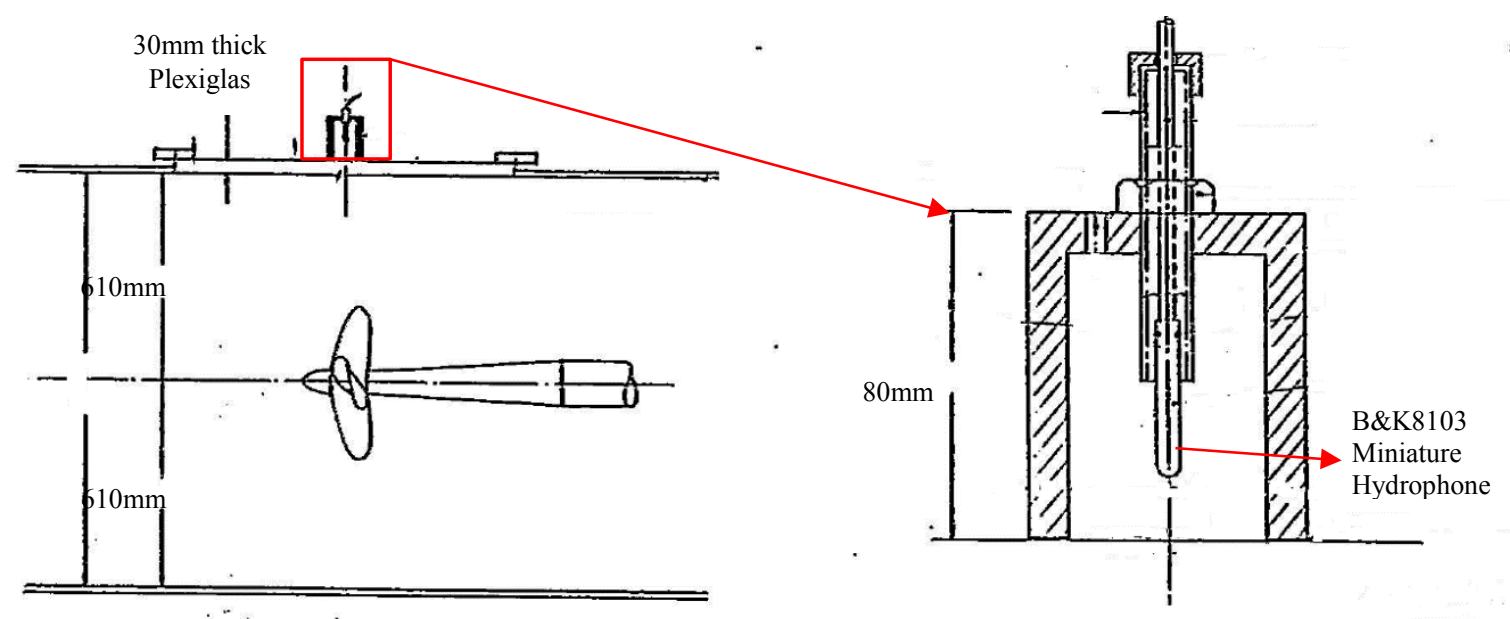

Figure 2 ECT hydrophone attachment configuration (View from top)

\subsection{UNIGE Cavitation Tunnel}

UNIGE cavitation tunnel is a Kempf \& Remmers closed water circuit tunnel, schematically represented in Figure 3. The tunnel has a square testing section of $0.57 \mathrm{~m} \times 0.57 \mathrm{~m}$, having a total length of $2 \mathrm{~m}$. The nozzle contraction ratio is $4.6: 1$, allowing to achieve a maximum speed of $8.5 \mathrm{~m} / \mathrm{s}$ in the test section.

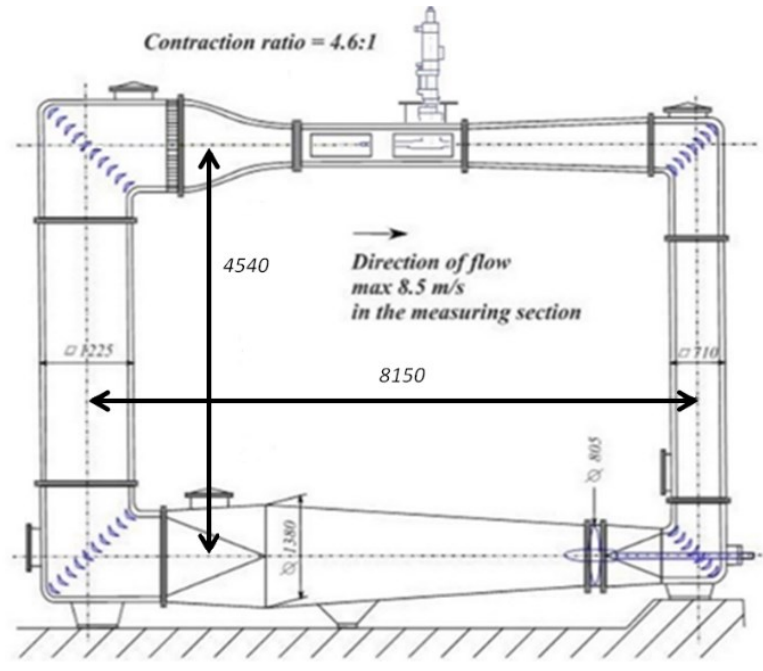

Figure 3: UNIGE cavitation tunnel

The vertical distance between the horizontal ducts is $4.54 \mathrm{~m}$ and the distance between the vertical ducts is $8.15 \mathrm{~m}$. 
The tunnel is equipped with a Kempf \& Remmers H39 dynamometer, able to measure thrust, torque and rate of revolution of the propeller. The maximum revolution rate achievable by the dynamometer is $3000 \mathrm{RPM}$ while maximum thrust and torque allowed on the are $100 \mathrm{Kg}(981 \mathrm{~N})$ and $500 \mathrm{Kg} \cdot \mathrm{cm}(49.45 \mathrm{Nm})$ respectively.

In accordance with ITTC guidelines the quality of the water in the tunnel is routinely checked during tests measuring the oxygen content through an ABB dissolved oxygen sensor model 8012/170, coupled with an ABB analyser model AX400. Three different oxygen levels have been considered for tests, as it will be shown in details in Section 6.2.

Water temperature during the whole campaign was between $17^{\circ} \mathrm{C}$ and $21^{\circ} \mathrm{C}$.

In the present campaign, tests have been carried out mounting the dynamometer with the propeller upstream and positioning with the propeller shaft in correspondence to the centre line of the tunnel test section. The same position has been adopted also for tests with inclined shaft, adjusting the height of the dynamometer.

Radiated noise measurements have been carried out with three hydrophones, namely one Reson TC4013 and two Bruel \& Kjaer type 8103. The hydrophones are coupled with Bruel \& Kjaer type 2635 charge amplifiers. Hydrophones configuration is shown in Figure 4.

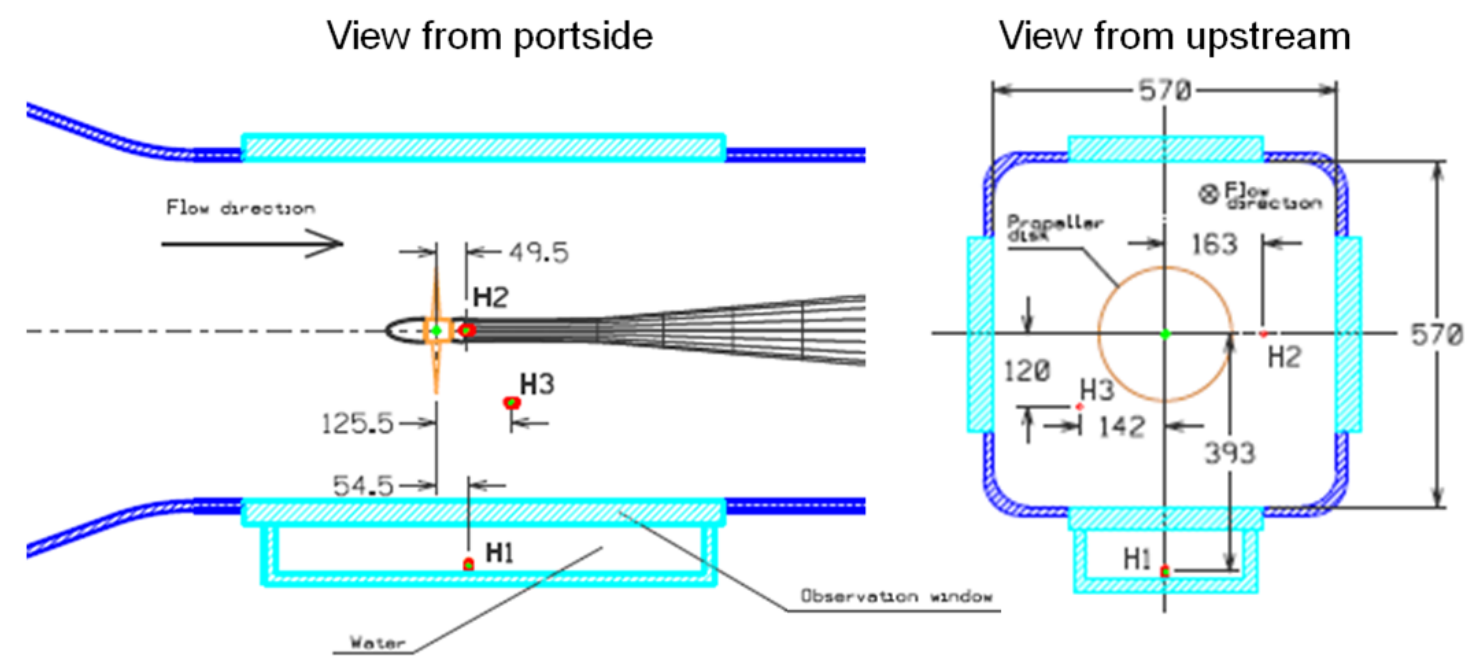

Figure 4: UNIGE cavitation tunnel setup

One hydrophone (H1) is placed in a rudimentary acoustic chamber consisting in a small tank made of PMMA (Plexiglas). This hydrophone is positioned on the observation window below 
the propeller and filled with water. The other two hydrophones are positioned inside the test section, downstream the propeller and outside the direct propeller slipstream.

For the radiated noise measurements, records made of $2^{21}$ samples are acquired with a sampling frequency of $200 \mathrm{kHz}$.

Following the standard procedures, usually adopted at UNIGE cavitation tunnel, propeller operational conditions are defined according to the identity of the thrust coefficient and cavitation number. Propeller revolution rate is selected as high as possible in order to maximize the Reynolds number, but keeping a certain margin with respect to the limits of the facility and, in particular, to the maximum forces allowed on the propeller dynamometer.

For a given revolution rate the flow speed in the cavitation tunnel is usually adjusted in order to achieve the required thrust coefficient.

The cavitation number adopted for cavitation similarity is usually computed with respect of propeller revolution rate, according to Equation 1.

$$
\begin{array}{ll}
\sigma_{N}=\frac{P_{\infty}+\rho g h-P_{V}}{0.5 \rho N^{2} D^{2}} & \text { Equation 1 } \\
& \\
\sigma_{V}=\frac{P_{\infty}+\rho g h-P_{v}}{0.5 \rho V^{2}} & \text { Equation 2 }
\end{array}
$$

This rotational cavitation number (Equation 1) may be derived from that based on the free stream velocity cavitation number (Equation 2) multiplying it by the square of the advance coefficient.

\subsection{Propeller Models}

Tests carried out are part of a round robin test campaign on the propeller of the University of Newcastle deep V catamaran The Princess Royal. Further details of the vessel can be found in (Atlar et al, 2013) In accordance with the general aims of the round robin campaign, different model propellers are considered, with slightly different dimensions and different manufacturers. 
The propeller is a five blade fixed pitch propeller, whose main characteristics and detailed design parameters for each section along the radius are reported in Table 1 and Table 2. The model propeller blade section offset is provided in Table 6 of Appendix A.

Table 1: Propeller main characteristics and particulars

\begin{tabular}{|l|c|}
\hline Full Scale Diameter [m] & 0.75 \\
\hline Pitch Ratio at 0.7R & 0.8475 \\
\hline Expanded Blade Area Ratio & 1.057 \\
\hline Number of blades & 5 \\
\hline
\end{tabular}

Table 2 "The Princess Royal” full-scale propeller main particulars

\begin{tabular}{|c|c|c|c|c|c|c|}
\hline $\mathrm{x}$ & Radius $\mathrm{mm}$ & $\mathrm{P} / \mathrm{D}$ nose-tail & Pitch nose-tail $(\mathrm{mm})$ & Chord $(\mathrm{mm})$ & Skew $(\mathrm{mm})$ & Thickness (mm) \\
\hline 0.2 & 75 & 0.8180 & 613.50 & 245.6 & -6.9 & 32.0 \\
\hline 0.25 & 93.75 & 0.8494 & 637.05 & 263.0 & -8.1 & 30.2 \\
\hline 0.3 & 112.5 & 0.8726 & 654.45 & 278.5 & -10.2 & 28.4 \\
\hline 0.4 & 150 & 0.8802 & 660.15 & 308.7 & -12.3 & 24.7 \\
\hline 0.5 & 187.5 & 0.8772 & 657.90 & 332.8 & -10.0 & 21.1 \\
\hline 0.6 & 225 & 0.8718 & 653.85 & 348.6 & -1.1 & 17.4 \\
\hline 0.7 & 262.5 & 0.8612 & 645.90 & 352.4 & 14.0 & 13.8 \\
\hline 0.8 & 300 & 0.8467 & 635.03 & 335.5 & 34.9 & 10.1 \\
\hline 0.9 & 337.5 & 0.8216 & 616.20 & 280.2 & 64.0 & 6.5 \\
\hline 0.95 & 356.25 & 0.7984 & 598.80 & 216.4 & 81.7 & 4.6 \\
\hline 1 & 375 & 0.7634 & 572.55 & 0 & 101.7 & 2.8 \\
\hline
\end{tabular}

The two model propellers have been manufactured with different scale factors, 3.5 and 3.41, resulting in model scale diameter of 0.214 and $0.22 \mathrm{~m}$ for the ECT and the UNIGE model propeller, respectively. The two models represent the same geometry; however, the slightly different dimensions and possible minor deviations could represent further sources of uncertainties to be regarded as part of the whole experimental campaign.

\subsection{Test matrix}

Tests in the two facilities have been carried out in two different stages of the round robin campaign. Actually, the propeller was tested at the Emerson cavitation tunnel prior to the official beginning of the round robin tests by the participants of the HTF Noise CoP. In these 
tests a very large set of operational conditions was considered, exploring eight values of advance coefficients (ranging from 0.4 to 0.75 ), three depressurization levels and seven inclination angles of the propeller shaft (ranging from $-9^{\circ}$ to $+9^{\circ}$ ). This allowed to define a complete grid of measurements, summarized in Table 3 , for the characterization of the effects of the inclined shaft on propeller cavitation and noise.

The performance of propellers are conventionally represented in terms of non-dimensional coefficients, i.e., thrust coefficient $\left(\mathrm{K}_{\mathrm{T}}\right)$, torque coefficient $\left(\mathrm{K}_{\mathrm{Q}}\right)$ and efficiency $\left(\eta_{\mathrm{O}}\right)$ and their variation with advance coefficients $(\mathrm{J})$ as given by Equation 3.

$$
\begin{array}{ll}
K_{Q}=\frac{Q}{\rho n^{2} D^{5}}, J=\frac{V_{A}}{n D}, K_{T}=\frac{T}{\rho n^{2} D^{4}}, \eta_{O}=\frac{J \times K_{T}}{2 \pi \times K_{Q}} \quad \text { Equation } 3
\end{array}
$$

Table 3 Systematic inclined shaft test matrix for tests conducted in Emerson Cavitation Tunnel

\begin{tabular}{|c|c|c|c|}
\hline & \multicolumn{3}{|c|}{ Test Type } \\
\hline & $\begin{array}{c}\text { Open water } \\
\text { performance tests }\end{array}$ & $\begin{array}{c}\text { Cavitation inception } \\
\text { tests and observations }\end{array}$ & Noise measurements \\
\hline $\begin{array}{c}\text { Shaft Incl. Angle } \\
\left({ }^{\circ}\right)\end{array}$ & \multicolumn{3}{|c|}{$0,3,6,9,-3,-6$ and -9} \\
\hline $\begin{array}{c}\text { Cavitation } \\
\text { Condition }\end{array}$ & $\begin{array}{c}\text { Atmospheric } \\
\text { condition } \\
\left(\sigma_{\mathrm{V}}=13.9\right)\end{array}$ & $\begin{array}{c}\text { Medium vacuum } \\
\text { condition }\left(\sigma_{\mathrm{V}}=8.1\right)\end{array}$ & $\begin{array}{c}\text { High vacuum condition } \\
\text { corresponding to vessel's } \\
\text { fully loaded condition } \\
\left(\sigma_{\mathrm{V}}=4.5\right)\end{array}$ \\
\hline J Range Tested & \multicolumn{2}{|c|}{$0.75,0.70,0.65,0.60,0.55,0.50,0.45,0.40$} \\
\hline
\end{tabular}

The angle convention adopted for the shaft inclination during the tests was that a positive angle was obtained when the dynamometer shaft (at the propeller end) was inclined in an upward direction with the blade tip at the 6 o'clock position moving towards the incoming flow as shown in Figure 5. The experiments were repeated at both negative and positive angles in order to account for the tunnel's speed profile at the propeller plane as shown in Figure 13. The propeller centre was always positioned on the tunnel centerline. 


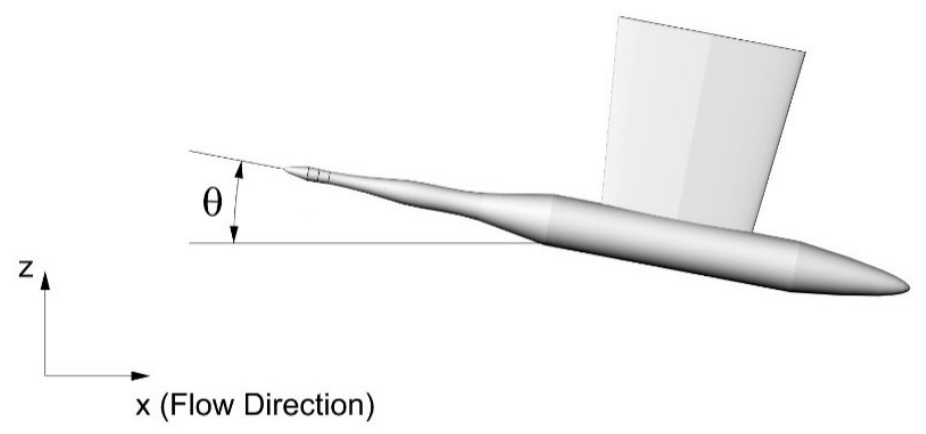

Figure 5 Angle convention for the conducted shaft inclination tests at ECT

From this grid, six operational conditions were then proposed for the round robin tests, considering conditions characterized by different extensions of cavitation, ranging from one condition close to inception to that with severe cavitation. In addition, it was decided to perform tests both in uniform inflow and with inclined shaft, but considering only one value for the inclination angle. In order to fit with the limitations of all the facilities involved in the round robin campaign it was necessary to limit the inclination angle to $5^{\circ}$. This value unfortunately was not included in the configurations tested at the Emerson cavitation tunnel, however it is rather close to one of them, i.e. the $6^{\circ}$ configuration.

These six operational conditions with the two shaft inclination configurations were considered for the Round Robin campaign. The operational conditions are reported in Table 4 together with the cavitation tunnel main functioning parameters.

Table 4: Round Robin test matrix

\begin{tabular}{|c|c|c|c|c|c|c|c|c|}
\hline Condition & $\mathrm{J}$ & $\sigma v$ & $\sigma_{N}$ & $N$ [RPS] & $\mathrm{K}_{\mathrm{T}}$ & $10 \mathrm{~K}_{\mathrm{a}}$ & $\mathrm{T}[\mathrm{Kg}]$ & $\mathrm{Q}[\mathrm{Kg} * \mathrm{~cm}]$ \\
\hline $\mathrm{C} 1$ & 0.4 & 13.9 & 2.22 & 45.5 & 0.242 & 0.341 & 119.2 & 369.5 \\
\hline $\mathrm{C} 2$ & 0.4 & 8.1 & 1.30 & 45.5 & 0.242 & 0.341 & 119.2 & 369.5 \\
\hline C3 & 0.4 & 4.5 & 0.72 & 45.5 & 0.242 & 0.341 & 119.2 & 369.5 \\
\hline $\mathrm{C} 4$ & 0.5 & 13.9 & 3.48 & 36.4 & 0.190 & 0.284 & 59.9 & 197.0 \\
\hline C5 & 0.5 & 8.1 & 2.03 & 36.4 & 0.190 & 0.284 & 59.9 & 197.0 \\
\hline C6 & 0.5 & 4.5 & 1.13 & 36.4 & 0.190 & 0.284 & 59.9 & 197.0 \\
\hline
\end{tabular}


Due to facility limitations it was not possible to reproduce the operational conditions maintaining the flow rate at $4 \mathrm{~m} / \mathrm{s}$ because this would result in a propeller thrust exceeding the maximum allowed by the H39 dynamometer.

Therefore, the operational conditions were set at lower shaft revolutions and consequently lower flow speed. In particular, 35 RPS were adopted for the propeller revolution rate, this means to reproduce conditions with the higher advance coefficient $(\mathrm{J}=0.5)$ with a flow speed rather close to the $4 \mathrm{~m} / \mathrm{s}$ of the reference functioning points. For what regards conditions with $\mathrm{J}=0.4$ propeller revolutions and flow rate are instead significantly reduced.

The modified operational conditions considered for tests at UNIGE cavitation tunnel are summarized in Table 5: Round Robin operational conditions modified for tests at UNIGE caviotation tunnel..

Table 5: Round Robin operational conditions modified for tests at UNIGE caviotation tunnel.

\begin{tabular}{|c|c|c|c|c|c|c|c|c|c|}
\hline Condition & $J$ & $\sigma V$ & $\sigma_{N}$ & $V_{A}[\mathrm{~m} / \mathrm{s}]$ & $\mathrm{N}[\mathrm{RPS}]$ & $\mathrm{K}_{\mathrm{T}}$ & $10 \mathrm{~K}_{\mathrm{Q}}$ & $\mathrm{T}[\mathrm{Kg}]$ & $\mathrm{Q}\left[\mathrm{Kg}{ }^{*} \mathrm{~cm}\right]$ \\
\hline $\mathrm{C} 1$ & 0.4 & 13.9 & 2.22 & 3.08 & 35 & 0.242 & 0.341 & 70.7 & 219.1 \\
\hline $\mathrm{C} 2$ & 0.4 & 8.1 & 1.3 & 3.08 & 35 & 0.242 & 0.341 & 70.7 & 219.1 \\
\hline $\mathrm{C3}$ & 0.4 & 4.5 & 0.72 & 3.08 & 35 & 0.242 & 0.341 & 70.7 & 219.1 \\
\hline $\mathrm{C} 4$ & 0.5 & 13.9 & 3.48 & 3.85 & 35 & 0.19 & 0.284 & 55.5 & 182.5 \\
\hline $\mathrm{C} 5$ & 0.5 & 8.1 & 2.03 & 3.85 & 35 & 0.19 & 0.284 & 55.5 & 182.5 \\
\hline C6 & 0.5 & 4.5 & 1.13 & 3.85 & 35 & 0.19 & 0.284 & 55.5 & 182.5 \\
\hline
\end{tabular}

The analysis of discrepancies between the facilities' procedures and their influence on final results is perfectly in line with the aims of the round robin campaign, thus it was decided to focus tests at UNIGE cavitation tunnel on the investigation of the influence of main tunnel 
functioning parameters on measured noise. These analyses, which are summarized in the following and described in details in section 6 , have been performed only for radiated noise measurements and, partially, cavitation observations. For the other tests only the standard setup was considered. This may result in small discrepancies between radiated noise measurements and inception tests. As an example, cavitation inception may be slightly different in the case of different oxygen levels. This aspect concerns especially for the condition close to inception condition (C4) which is, as it will be shown, more sensitive to small variations of the tunnel operational conditions.

According to this, measurements at the six considered operational conditions have been carried out with different propeller revolution rate, namely 30 and 35 RPS and other revolution regimes have been considered only for preliminary analysis. In addition, the sensitivity of measurements with respect to the cavitation number and thrust coefficient has been analysed by considering additional tests defined as small percentage variation of the functioning parameters ( $\pm 5 \%$ for the thrust coefficient and $\pm 10 \%$ for the cavitation number). This results in defining four additional test conditions for each main functioning points, the analysis of these conditions allows also to roughly deal with the problem of measurement uncertainty, as suggested by ITTC (2002).

Finally, also different water quality, namely for different levels of dissolved oxygen, have been considered for radiated noise measurements. This is partially required by the particular characteristics of the propeller operational conditions considered, ranging from atmospheric condition with likely limited cavitation to high vacuum with likely severe cavitation. Actually the optimal water quality for such conditions is not the same, as a consequence three oxygen levels have been defined to better fit with the characteristics of the actual operational conditions tested. Of course, this allowed also to test the same operational condition with two or three oxygen levels, investigating the influence of this parameter on radiated noise tests in correspondence to significantly different operational conditions and cavitation extensions.

\section{Propeller performance tests}

Propeller open water performance characteristics (thrust and torque) are usually measured in towing tank, according to standard ITTC (2008) procedures. However, it is usual practice to 
carry out this kind of measurements also at the cavitation tunnel, mainly as a preliminary measurement from which the reference curves describing the propeller functioning in the tunnel for the successive cavitation tests are derived.

This was done also in present case by both facilities with respective propellers. From this point of view it has to be remarked that, while for the ECT model propeller the towing tank measurements were available, for the UNIGE model propeller, open water tests in towing tank were not carried out.

Propeller thrust and torque were measured consistently with the test procedures adopted by the two involved facilities for the following tests. Thus tests at the ECT were performed keeping the flow rate constant at $4 \mathrm{~m} / \mathrm{s}$ and varying the propeller revolution rate. At UNIGE cavitation tunnel fixed propeller revolution rate was considered and the advance coefficient was varied by changing the tunnel flow speed. In accordance with the standard practice different revolution rates were considered in order to check measurements dependency on Reynolds number. Of course, revolution rates adopted for noise tests were included.

Results of measurements carried out without shaft inclination at the ECT were compared with measurements from towing tank in Varna by Bulgarian Ship Hydrodynamic Center (BSHC) and results are presented in Figure 6. The measurement of the propeller performance by fixing the inflow speed and varying the propeller revolution rate showed to underestimate the thrust in the high advance coefficient region. The reason behind the difference may be attributed to a combined effect of inherent low Reynolds Number in this particular region as well as the measured values corresponding to the lower range of the utilized dynamometer at ECT.

As it can be seen in Figure 6, some discrepancies are present, these are in general always present when comparing the open water test results from towing tanks and cavitation tunnels. However, in the present case, the main responsible for the discrepancies may be related to the adoption of constant tunnel speed while varying the propeller revolutions which cause the Reynolds number to vary from $4.03 \times 10^{6}$ to $8.22 \times 10^{6}$ as defined in Equation 4 .

$$
R e=\frac{C_{0.7 R} \sqrt{V^{2}+(0.7 \pi n D)^{2}}}{v}
$$


However, it has to be underlined that this measurement does not represent a general estimate of propeller open water performances but the reference curves for cavitation tunnel tests, thus discrepancies do not represent a problem for the successive tests.

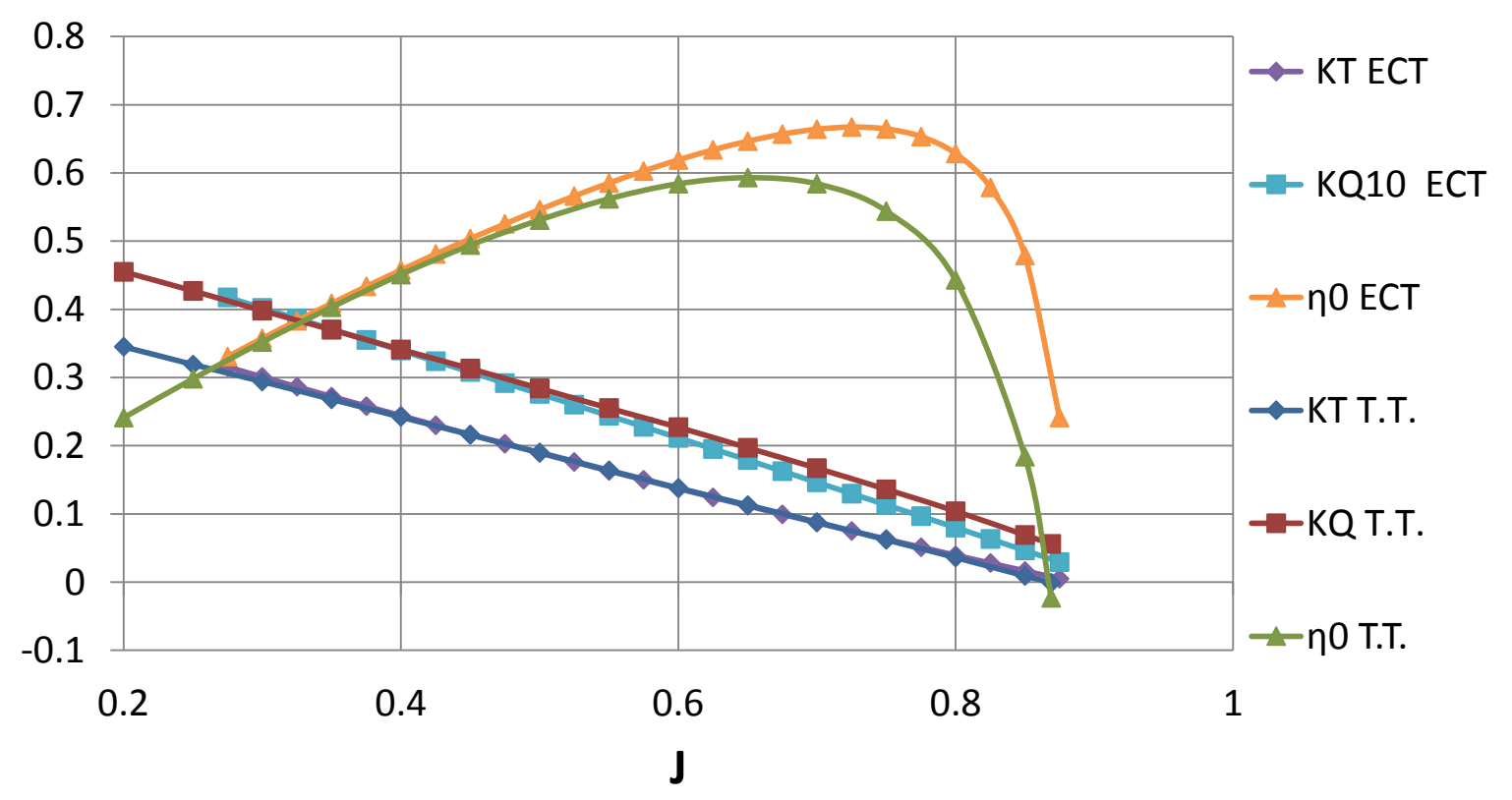

Figure 6: Open water characteristics, comparison between ECT and towing tank.

Tests performed at UNIGE cavitation tunnel allowed investigation of the effect of the Reynolds number on delivered thrust and torque for values ranging from $7.6 \times 10^{5}$ to $1.9 \times 10^{6}$. These results are summarized in Figure 7 while Figure 8 reports the comparison with towing tank results considering cavitation tunnel tests at 30 RPS. According to the presented results, the influence of shaft revolution rate on the open water characteristics of UNIGE propeller is practically negligible in the considered range. Comparing results with the open water tests some differences are present, consisting mainly in a shift of the advance coefficient of the $2 \%$. The presence of this shift with respect to towing tank results is in line with previous experiences at the UNIGE cavitation tunnel. 


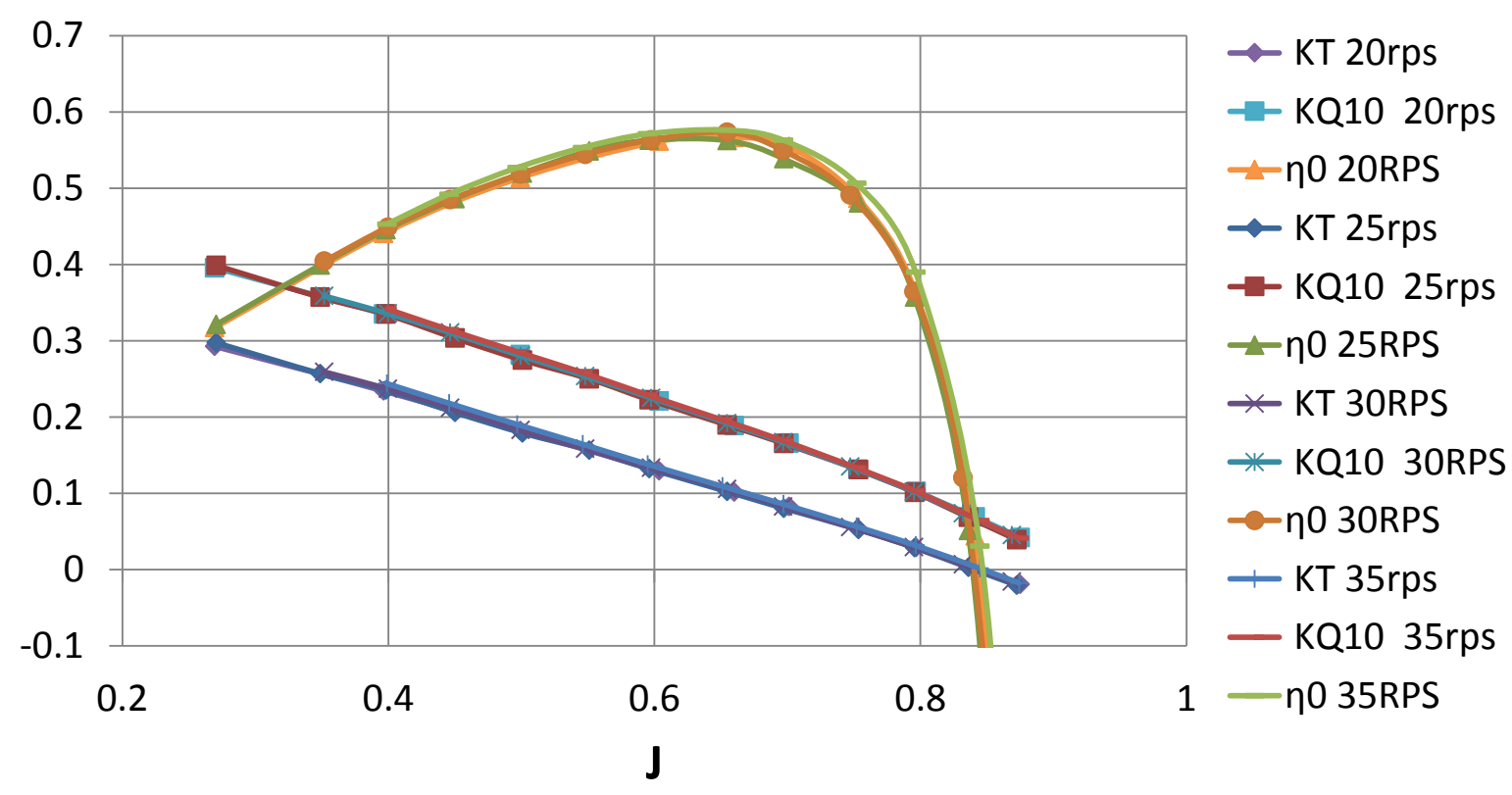

Figure 7: Propeller open water characteristics at different revolution rates .

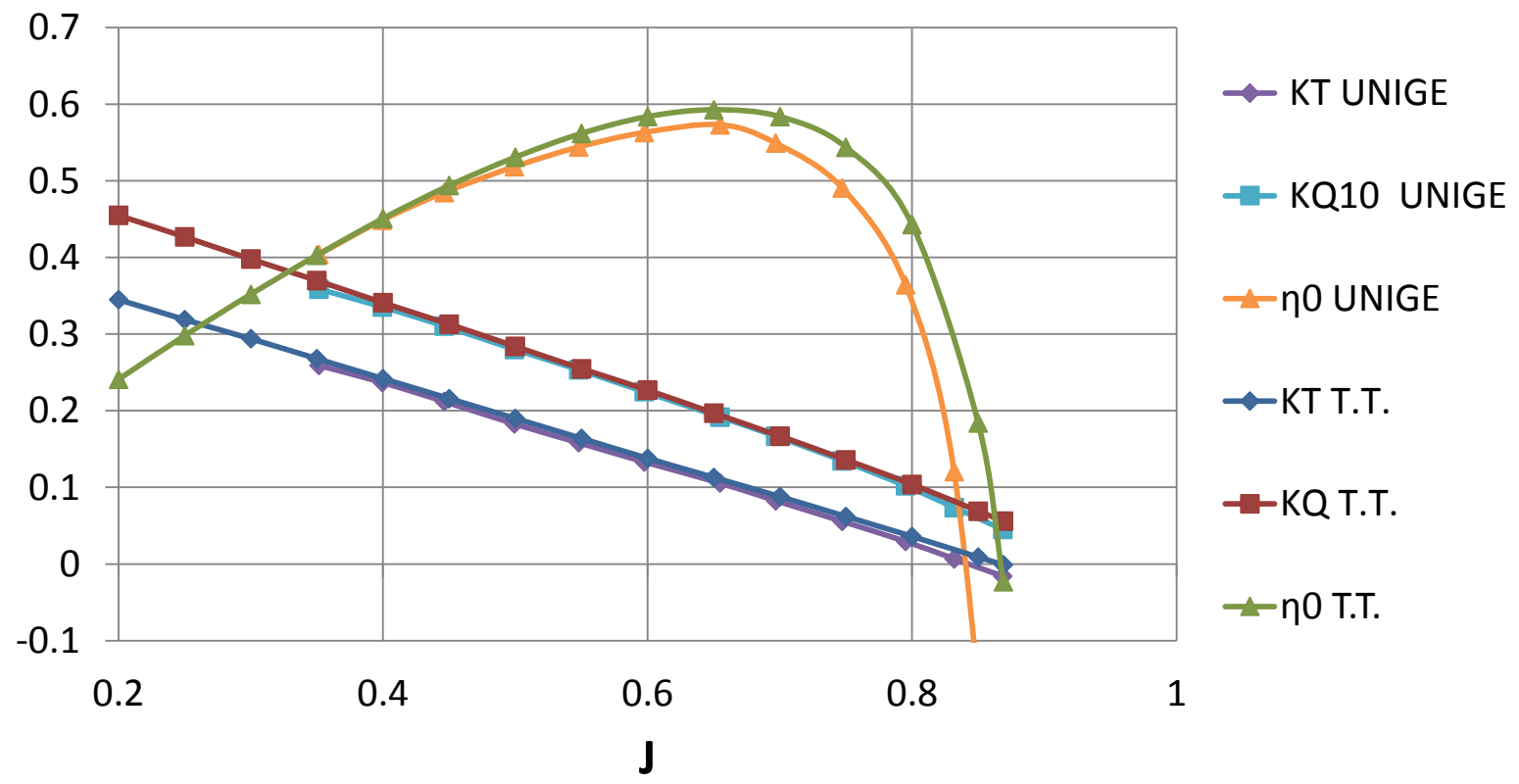

Figure 8: Propeller open water characteristics, comparison UNIGE cavitation tunnel and towing tank (two different propeller models).

\section{Cavitation Inception measurements}

In this section, inception tests carried out in the two facilities and respective results are compared. The round robin campaign representing the presented activities was not focused on the cavitation inception assessment. These measurements have been thus carried out only to 
obtain a more complete characterization of propeller cavitation behaviour as well as to collect useful information for better understanding of the noise results.

Nevertheless, the comparison of inception measurements provides a further interesting insight into general issues regarding cavitation testing and interpretation of model scale results.

Inception tests at the UNIGE cavitation tunnel are generally carried out fixing propeller revolution rate and flow speed, and lowering the tunnel static pressure until cavitation appears. The shaft rate is set as high as possible, in accordance with the already described equipment limitations. A further constraint on the shaft rate for cavitation tests is given by the need to avoid cavitation at atmospheric conditions, since it is not possible to increase the tunnel pressure above the atmospheric pressure.

Usually, for the operational conditions of major interest, tests are repeated, at least five times, in order to check the repeatability of cavitation inception measurement and to increase the reliability of results. In addition, cavitation inception is measured for a rather wide range of values of the thrust coefficient in order to obtain a complete characterization of the propeller and to further verify the consistency of results considering the global coherence of the measured inception curves.

Finally, the desinence of cavitation is measured starting from a condition with developed cavitation, namely tip vortex connected to the blades, and slowly increasing the static pressure until cavitation disappears.

Inception tests at ECT are carried out by increasing the propeller revolution speed while the inflow and tunnel pressure is kept constant. The determination of the inception and desinence points are made both by observations with bare eye as well as with the aid of high-speed video captures. Following the determination of the inception point, propeller revolution speed is increased until cavitation is well developed. This is then followed by gradual reduction of the propeller RPM for the determination of desinence point. Figure 9 is a representative capture of cavitation inception and desinence points. 


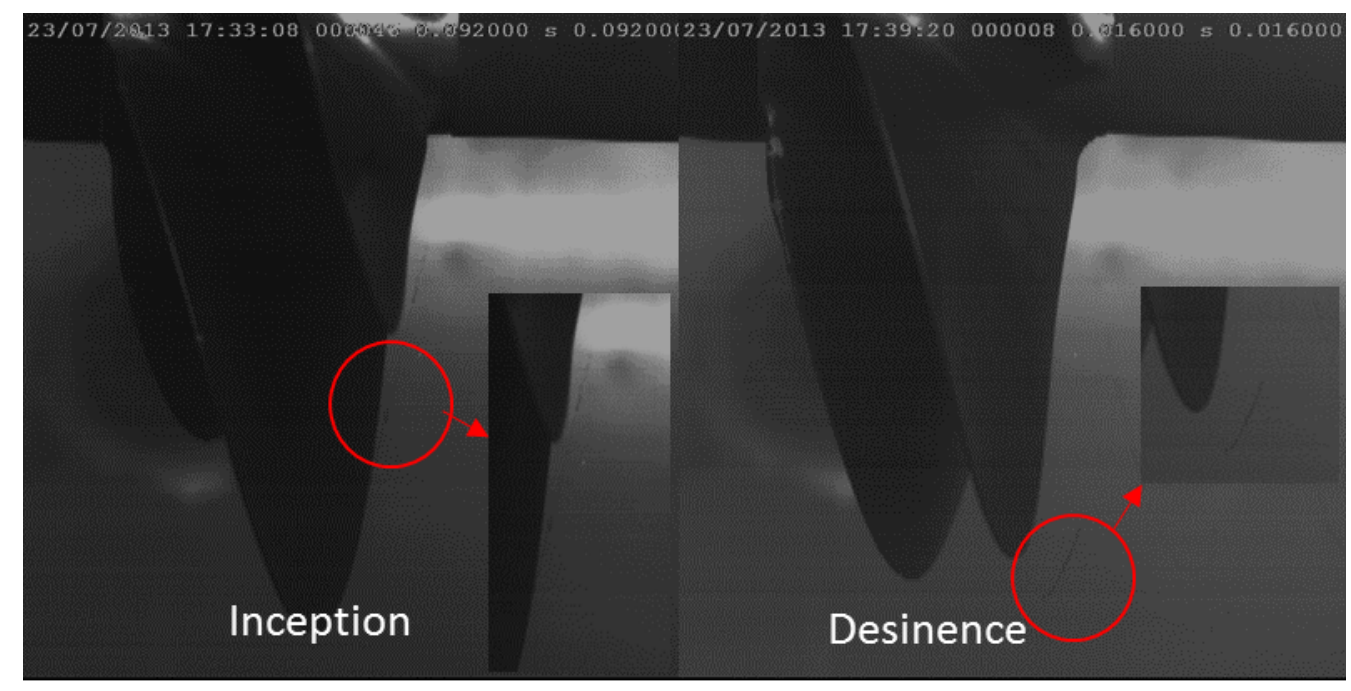

Figure 9 Images of the cavitation inception and desinence points for propeller model at $0^{\circ}$ inclination angle under high vacuum condition

Results are summarized in Figure 10 and Figure 11 reporting for each measurement the inception index at the corresponding value of the thrust coefficient.

Results obtained with different shaft inclinations are directly compared in Figure 12, considering only inception curves for clarity.

As known, the inception of tip vortex cavitation is influenced by the Reynolds number following the Mc Cormick law (McCormick, 1962). This may cause small differences between tests carried out at different shaft rates, therefore in principle a fair comparison between inception points could be done only at same revolution rate. Shaft rate differences in model scale testing have a limited impact on Reynolds number and inception, nevertheless it was preferred to take into account this phenomenon scaling all inception and desinence indices to a reference shaft rate (35RPS) according to the above mentioned scaling law, reported in Equation 5.

$$
\frac{\sigma_{i}(n)}{\sigma_{i}\left(n_{r e f}\right)}=\left(\frac{R e(n)}{\operatorname{Re}\left(n_{r e f}\right)}\right)^{0.34}
$$

In the plots both inception and desinence are considered, in addition, for UNIGE tests in correspondence to the main functioning conditions, also the $95 \%$ confidence interval is reported allowing to appreciate also the variability of the results of repeated tests. 


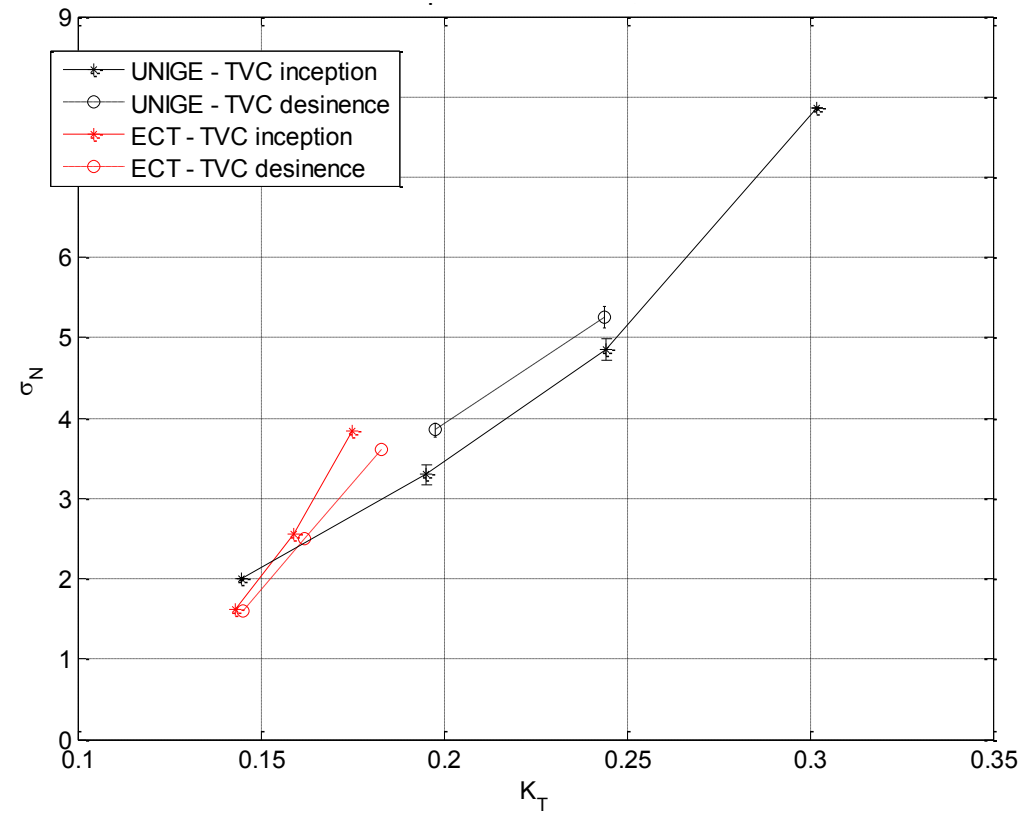

Figure 10: Cavitation inception results, uniform inflow.

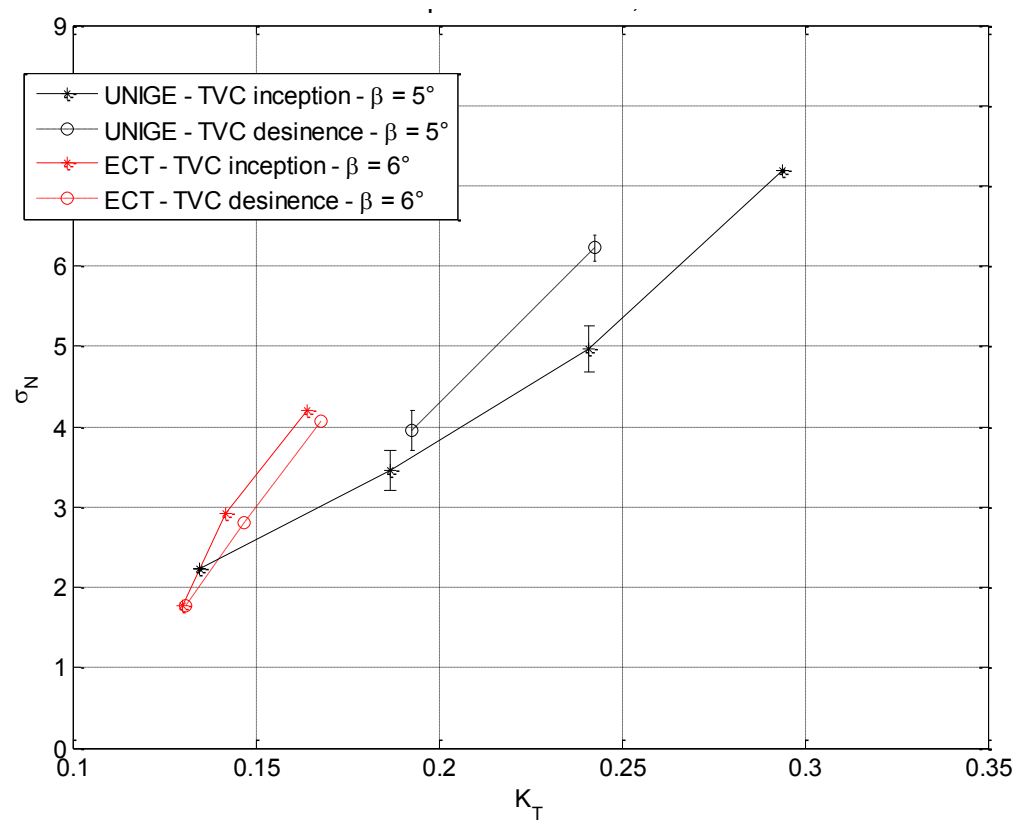

Figure 11: Cavitation inception results, inclined shaft. 


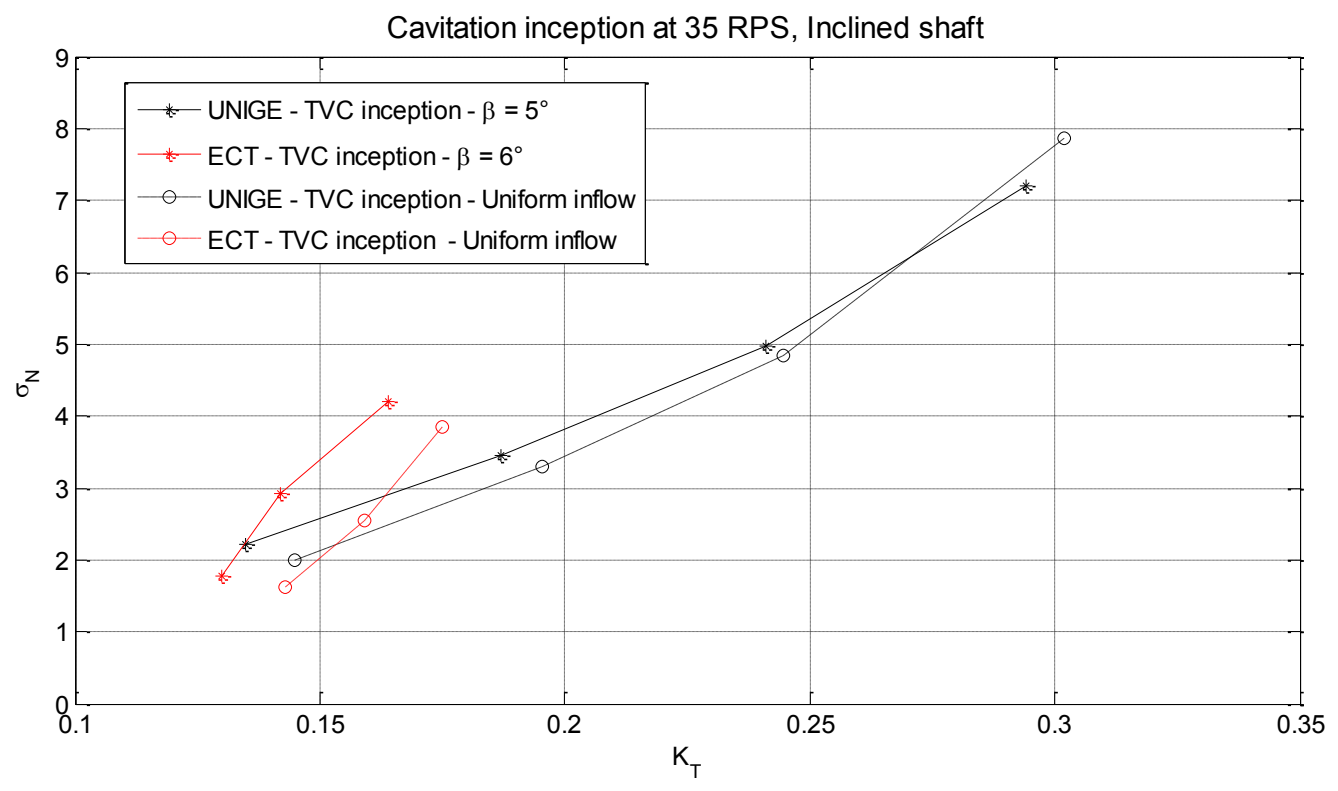

Figure 12: Cavitation inception results, effect of shaft inclination

As a first comment, the curves measured with and without shaft inclination look in all cases rather similar. In particular, the effect of the shaft inclination seems larger in the case of ECT results, even if it is clearly visible also for UNIGE tests, except for the highest loading condition considered. This gives the opportunity to point out a possible issue related to the water quality for tests at UNIGE cavitation tunnel. Actually, the oxygen content adopted for inception tests ( $5 \mathrm{ppm}$, about $55 \%$ of saturation at atmospheric pressure) represents a typical intermediate level, selected in order to have a good compromise for the different conditions considered. However, this means that, moving to extremely overloaded conditions, for which cavitation occurs at rather high cavitation number, the nuclei content may be not sufficient and consequently measured inception indices are likely to be underestimated and generally slightly less reliable (Chahine, 2004).

This problem seems not present for the operational conditions close to those selected for the round robin tests. Actually, the dispersion of measured inception indices is not severe and generally in line with typical results at UNIGE cavitation tunnel.

From this point of view, it has to be remarked that two blades were found to cavitate earlier. This behaviour is deemed to be related to small imperfections or damages at blades tip and in general the inception is defined when cavitation appears on the other blades, neglecting the first two blades, in accordance with (ITTC, 2011). Such anomalies are not rare when dealing 
with the inception of tip vortex cavitation, as reported as an example in (Pennings et al., 2015a), even with model propellers manufactured according to ITTC standards (ITTC, 2011).

Coming back to the comparison between results obtained in the two facilities, inception indices are similar for the lower loading conditions while they appear to diverge moving to higher thrust coefficient. Actually inception and desinence curves obtained at ECT are characterized by higher slope than those measured at UNIGE. For the purposes of the round robin campaign it is of great interest to investigate the reasons for these discrepancies.

Water quality influences significantly cavitation inception and desinence, especially in the case of tip vortex cavitation. As already mentioned, inception tests carried out at the University of Genoa may suffer of lack of cavitation nuclei at the higher loading conditions, likely resulting in under-predicted inception indices. However, this problem should not affect measurements for those conditions for which also ECT results are available.

Differences observed seem remarkable especially keeping in mind that tests are carried out with relatively simple and easily repeatable configurations, i.e. uniform inflow and inclined shaft, without wire screens or dummy models. However, this brings to the attention possible effects of propeller inflow; actually, also with considered configurations the speed profile may vary from one facility to another. A further aspect, regarding propeller inflow, which may influence significantly cavitation inception is the turbulence intensity, as discussed in (Korkut et al., 2000; Korkut and Atlar, 2002). Speeds profiles are reported in Figure 13. For what regards average turbulent intensity, values are $1.7 \%$ at UNIGE and $1.37 \%$ at ECT. 

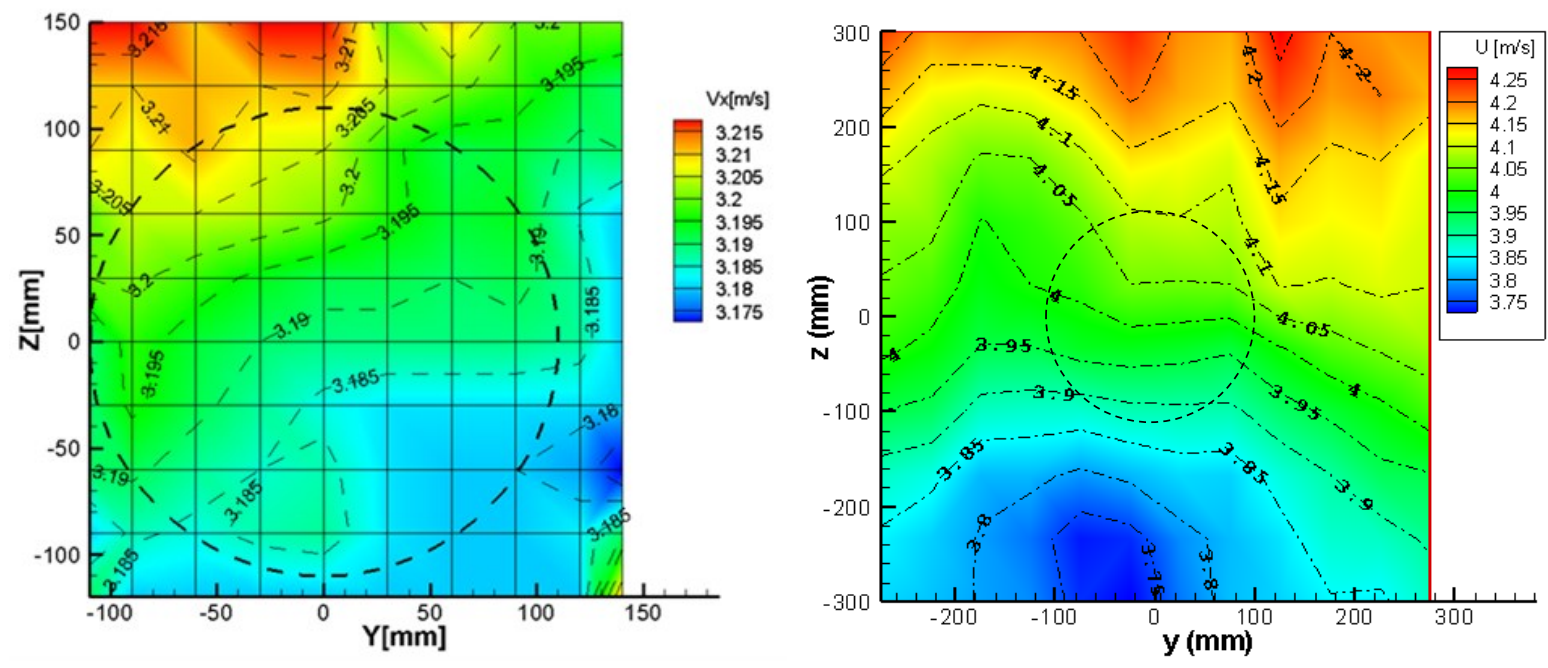

Figure 13: Axial Speed profiles of the UNIGE (left) and ECT (right)

Areas reported in the plots are different, partially because of the different dimensions of the facility and respective observation windows. Velocities are different too, because at UNIGE lower revolution rate has been adopted for the $\mathrm{J}=0.4$ conditions and LDV surveys have been made for the advance speed corresponding to that condition. Anyway, both speed profiles feature some speed variations, with values increasing from the lower part towards the upper. However, focusing on the area corresponding to propeller disk deviations are reduced: about $1 \%$ in the case of UNIGE cavitation tunnel, about $5 \%$ at ECT.

To take into account possible effects of the speed profile, measurements at ECT have been carried out inclining the shaft in both directions, thus inception curves are available also for $6^{\circ}$. These results are reported, together with those already shown, in Figure 14. As it can be seen, the two opposite inclinations result in rather different cavitation inceptions. In addition, even if the slope of the curves are still slightly different, the agreement between ECT and UNIGE results is significantly better if data obtained with $\beta=-6^{\circ}$ are considered. 


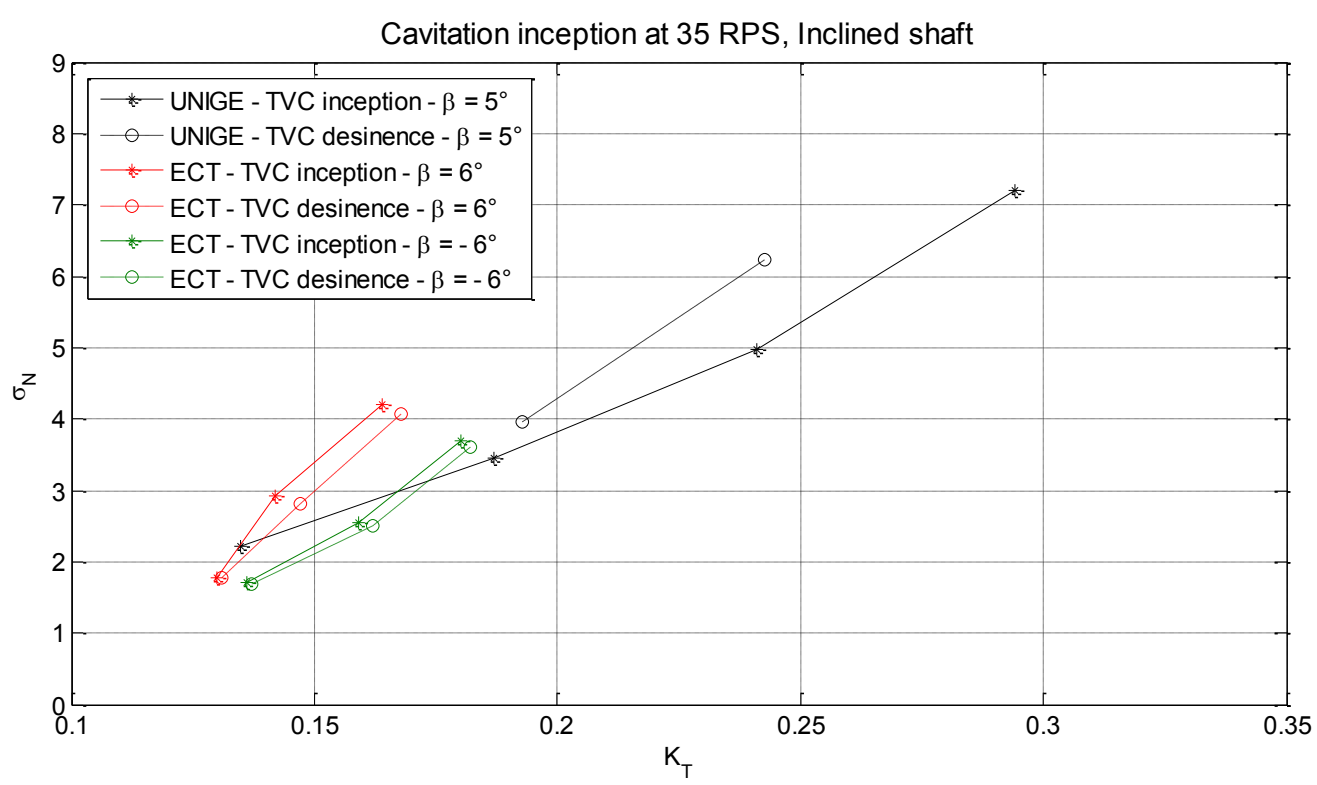

Figure 14: Cavitation inception results, inclined shaft.

Further explanations for the discrepancies in inception tests may come from the differences in the operational procedures adopted by the two institutions and from the different definitions adopted for inception and desinence assessment. As an example, the definition of desinence adopted at ECT corresponds to the point at which the tip vortex is no more connected to the blade, see Figure 9, while at UNIGE cavitation tunnel the desinence is defined as the condition for which cavitation is no more present. This easily explains why ECT desinence indices are slightly lower than inception indices while at UNIGE desinence always occurs at cavitation numbers higher than inception.

These dynamics are then consistent with definitions and with the theoretical dynamics of cavitation (Kuiper, 2001); however the gap between inception and desinence at UNIGE may be also partially intensified by a sort of hysteresis typical of this cavitation tunnel. Actually, due to the limited height of the facility, free bubbles in the flow are not completely destroyed while passing in the lower part of the tunnel. Due to this, the amount of free bubbles during a cavitation test may increase, causing this hysteresis phenomenon. 
Finally, during inception tests, the cavitation number is lowered acting on the tunnel static pressure at UNIGE, on shaft revolution rate at the ECT. This may cause cavitation inception (and desinence) to occur with different dynamics. As an example at UNIGE inception tests may sometime suffer of a sort of delay in the assessment of the inception. This delay is usually fixed considering repeated tests, ad hoc cavitation observations and, in general, considering both inception and desinence curves. This has been done also in present campaign thus the effect of this kind of problems on results should be negligible, according to previous experiences, however it may represent a further source of discrepancies with the ECT.

\section{Cavitation observations}

ECT cavitation observation techniques included high-speed video recording with continuous lighting or with triggered stroboscopic lighting, still imaging using DSLR and flash-cam video with stroboscopic lighting.

The most commonly used observation technique for the cavitation tunnel tests are the high speed camera recordings aided with stroboscopic lighting synchronized with shaft position signal as shown in Figure 15. This enables freezing the image due to the triggered flash provided by the strobe lights. Stroboscope lighting also accommodates the still imaging with a DSLR camera by reducing the shutter speed in order to fit the image acquisition into the short bursts of the strobe flash. 


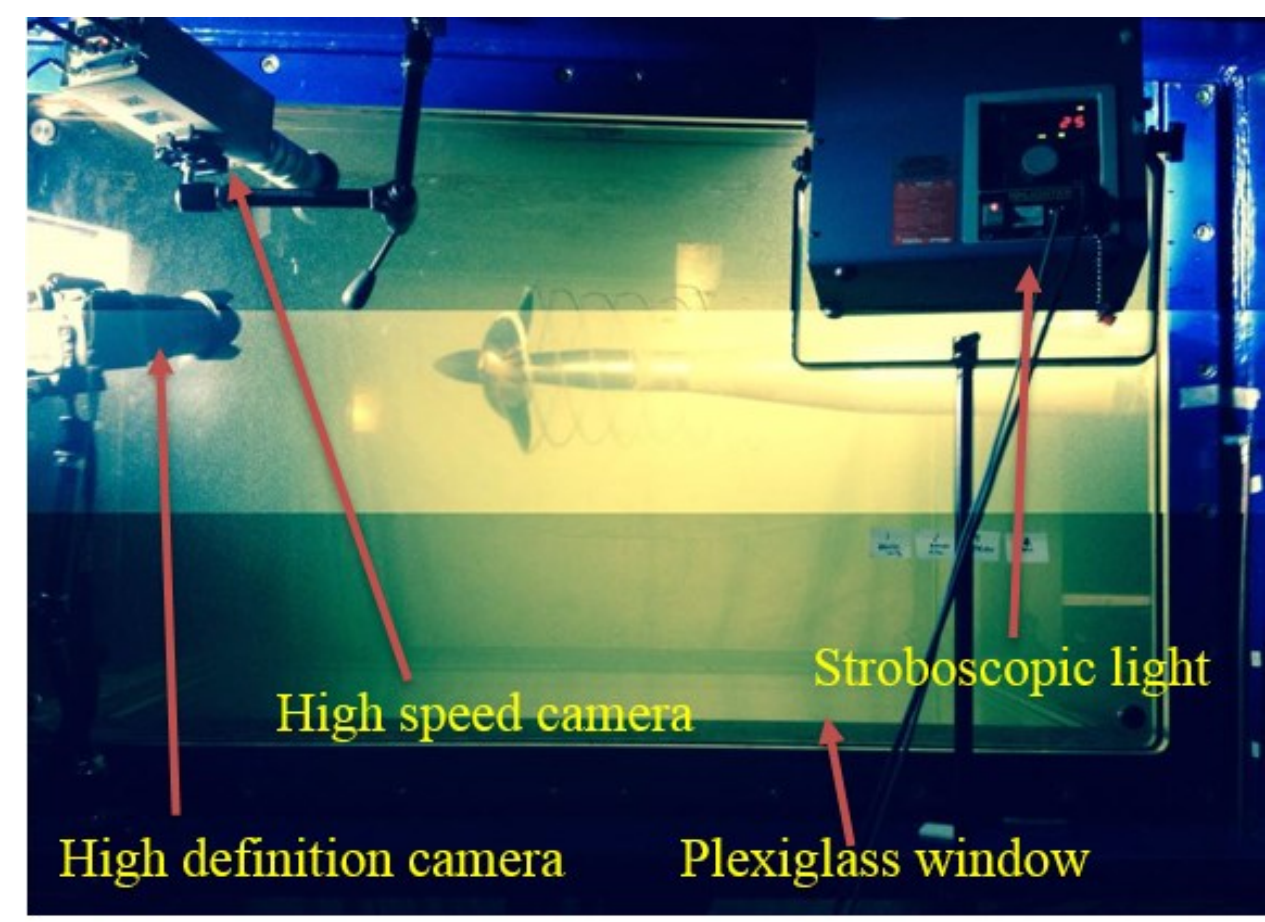

Figure 15 Cavitation observation using stroboscopic lighting

Whilst observing blade images synchronized with the shaft speed simplifies the adjustment of the cameras, in non-uniform inflow it is essential to record information on cavitation dynamics. Therefore, Nanosense high-speed cameras were also used at $5000 \mathrm{fps}$ in a continuous recording mode for the non-uniform inflow conditions. A high intensity light source was obtained from Plasma-Lite continuous light, to allow a high acquisition rate for the high-speed cameras.

Cavitation observations at UNIGE cavitation tunnel are carried out adopting three Allied Vision Tech Marlin F145B2 Firewire Cameras, with a resolution of 1392 x 1040 pixels and a frame rate up to $10 \mathrm{fps}$. The lighting is given by a mobile stroboscopic system synchronized with propeller shaft.

The three cameras are positioned as follows:

- Cam0: Positioned on starboard, pointing toward the propeller pressure side and its wake. This setup is usually adopted to capture pressure side cavitation; moreover, cavitating vortices may be also captured with the help of light contrast.

- Cam1: Positioned on portside, pointing to the suction side of the blade at $90^{\circ}$ and partially other angular positions.

- Cam2: Positioned above the propeller, focusing the whole propeller suction side. 
In the majority of cases the view from above is the most effective in describing cavitation pattern on the propeller, thus only pictures from Cam2 are presented. Cam1 is considered too, only for close to inception cavitation with inclined shaft, since in this case it could be useful to focus on cavitation when it first occurs. Finally, Cam0 is disregarded since pressure side cavitation was not observed and the other cameras are already sufficient to identify the presence of tip vortex cavitation.

Passing to the analysis of results, cavitation extensions measured at the ECT and at the UNIGE cavitation tunnel are compared in following figures provided within Table 5.

Reported photographs show in general a very good correlation between cavitation extensions observed in the two facilities. Cavitating phenomena are always analogous, consisting in tip vortex cavitation and suction side sheet cavitation of different extensions depending on the functioning condition.

Cavitation extensions seem very similar also, for what regards the dimensions of the bubbles:

- Conditions C1: Regular and well developed tip vortex is visible, with suction side sheet cavitation from about $0.8 \mathrm{R}$.

- Condition C2: This is the condition with the largest tip vortex extensions, vortices show a ribbon like shape and sheet cavitation is present from about $0.7 \mathrm{R}$.

- Condition C3: Large sheet cavitation from about 0.5R. The tip vortex is almost disappeared, with only cloudy structures shed by the sheet cavity at tip while a second vortex (smaller than the one present for condition C2), is present at the trailing edge of the sheet cavity.

- Condition C4: In this condition, the only cavitation phenomenon visible is a weak tip vortex.

- Condition C5: A small but stable tip vortex is present, together with sheet cavitation from about $0.9 \mathrm{R}$.

- Condition C6: Significant tip vortex cavitation with ribbon line shape, sheet cavitation from about $0.85 \mathrm{R}$. 
As it can be seen, in both facilities, the effect of shaft inclination on cavitation patterns is limited, only in the case of condition C4, cavitating vortices appears more often at the $90^{\circ}$ position, otherwise cavitation extensions at different angular positions are always very similar.

Table 6 Cavitation observation comparisons between ECT and UNIGE

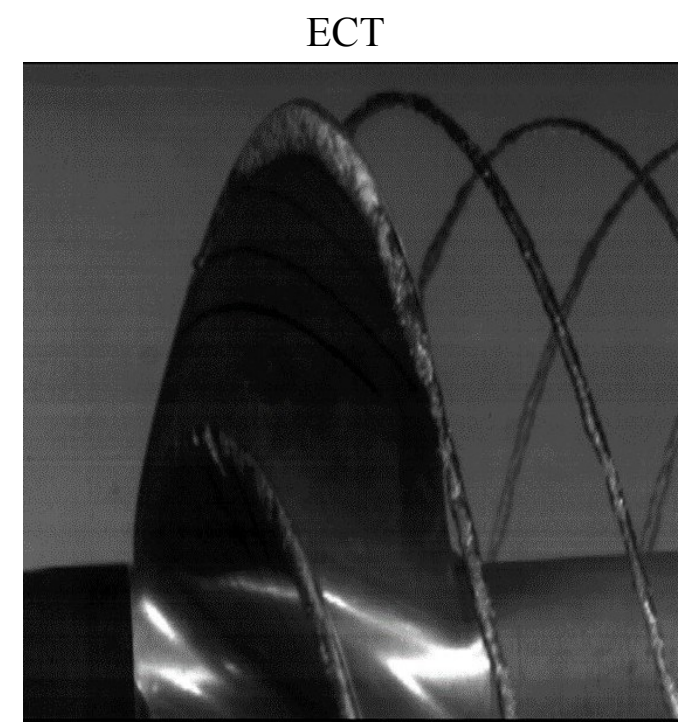

$6^{\circ}$ inclination angle

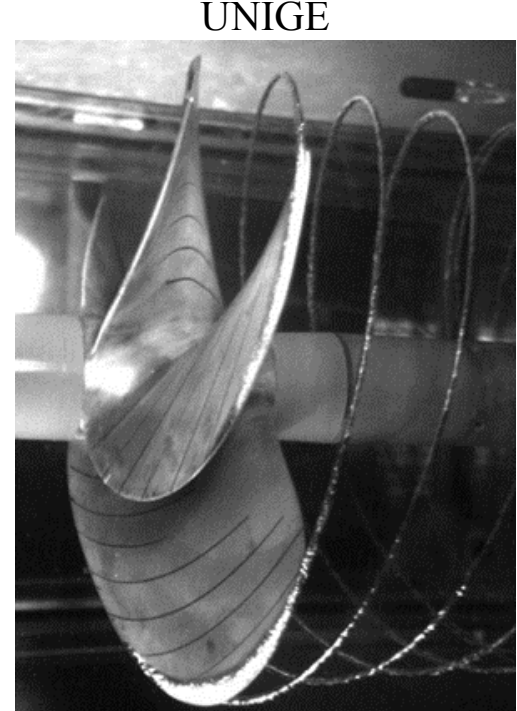

$5^{\circ}$ inclination angle

$\mathrm{C} 1: \mathrm{K}_{\mathrm{T}}=0,242, \mathrm{~J}=0.4, \sigma_{\mathrm{n}}=2.22$
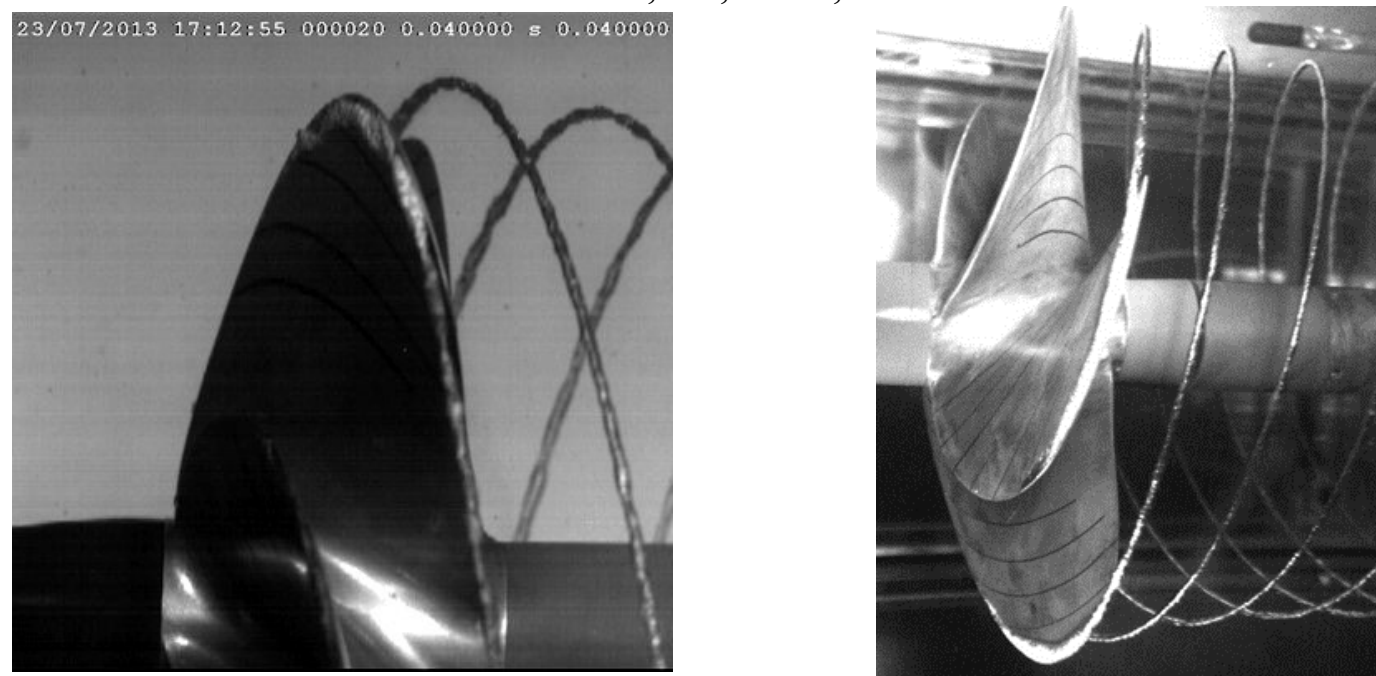

$\mathrm{C} 1: \mathrm{K}_{\mathrm{T}}=0,242, \mathrm{~J}=0.4, \sigma_{\mathrm{n}}=2.22,0^{\circ}$ inclination angle 
ECT

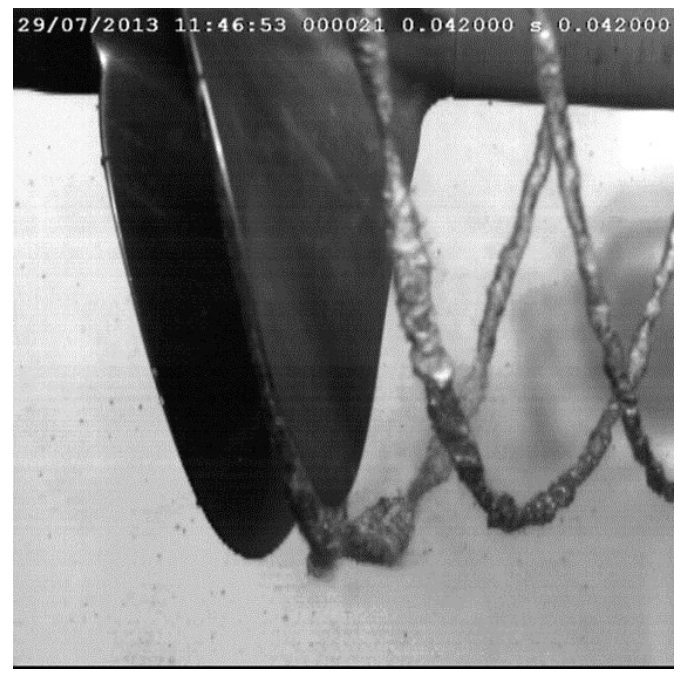

$6^{\circ}$ inclination angle
UNIGE

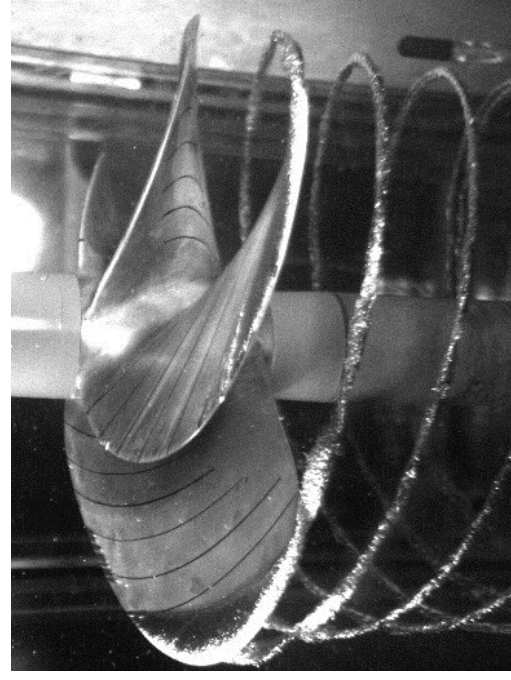

$5^{\circ}$ inclination angle

$\mathrm{C} 2: \mathrm{K}_{\mathrm{T}}=0,242, \mathrm{~J}=0.4, \sigma_{\mathrm{n}}=1.3$
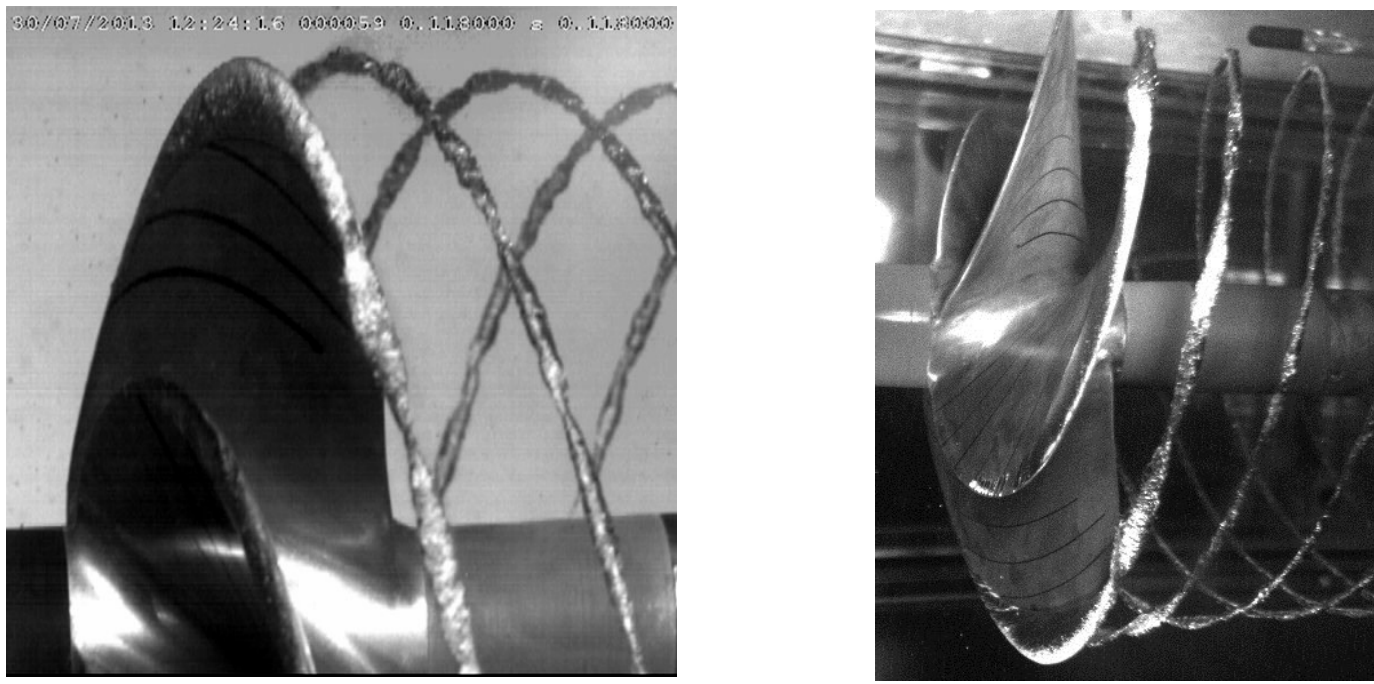

$\mathrm{C} 2: \mathrm{K}_{\mathrm{T}}=0,242, \mathrm{~J}=0.4, \sigma_{\mathrm{n}}=1.3,0^{\circ}$ inclination angle 


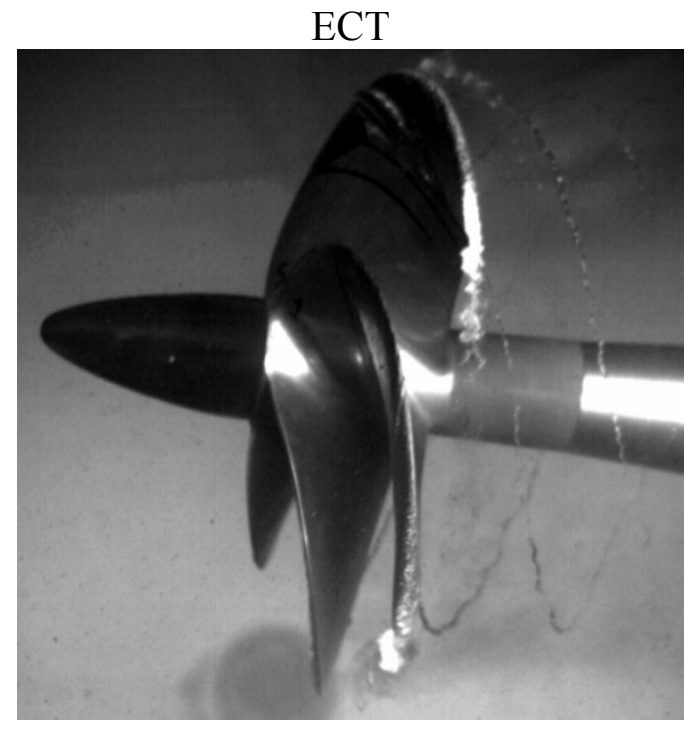

$6^{\circ}$ inclination angle

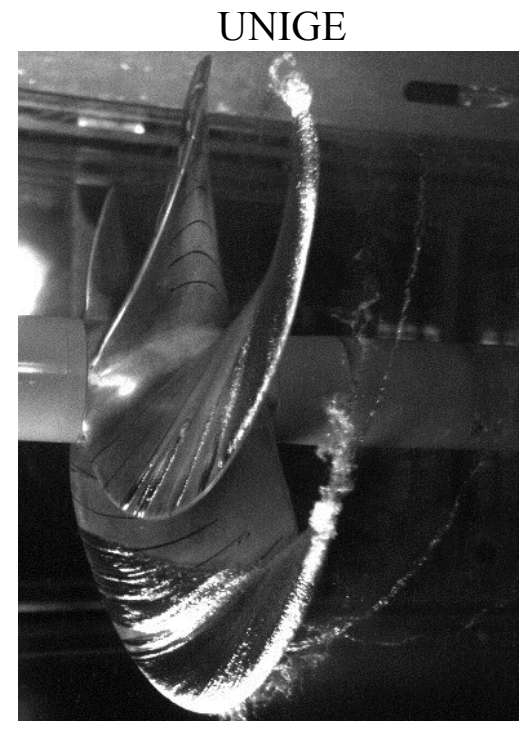

$5^{\circ}$ inclination angle

C3: $\mathrm{K}_{\mathrm{T}}=0,242, \mathrm{~J}=0.4, \sigma_{\mathrm{n}}=0.72$
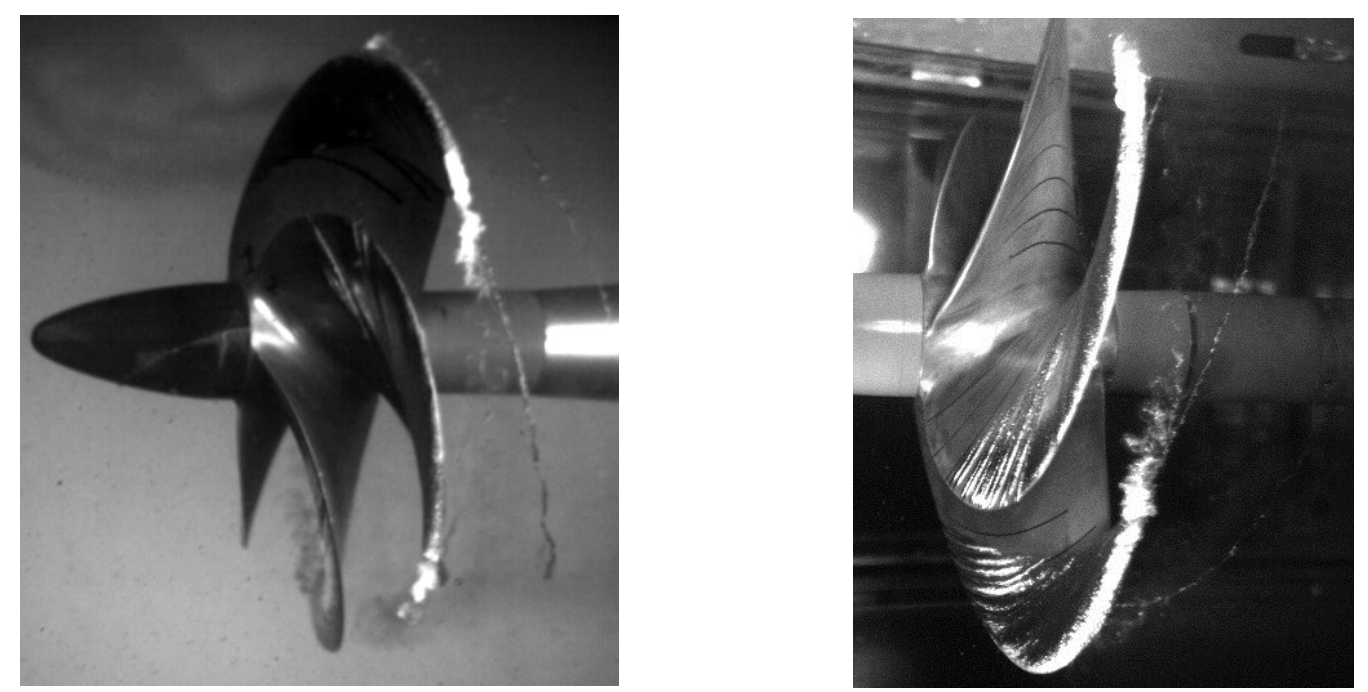

C3: $\mathrm{K}_{\mathrm{T}}=0,242, \mathrm{~J}=0.4, \sigma_{\mathrm{n}}=0.72,0^{\circ}$ inclination angle 
ECT

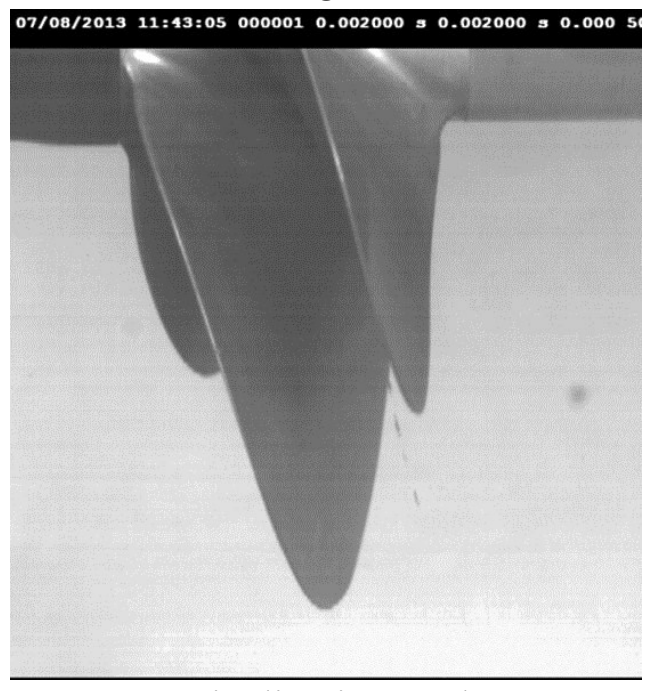

$6^{\circ}$ inclination angle

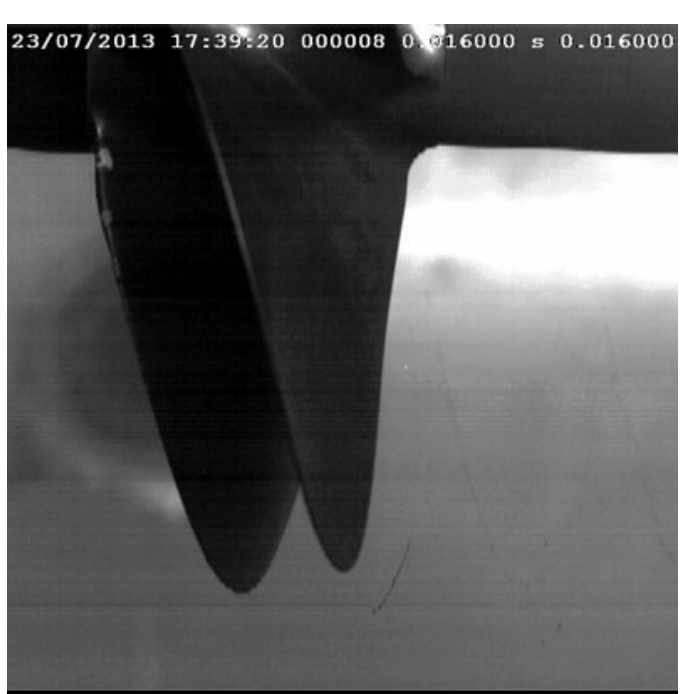

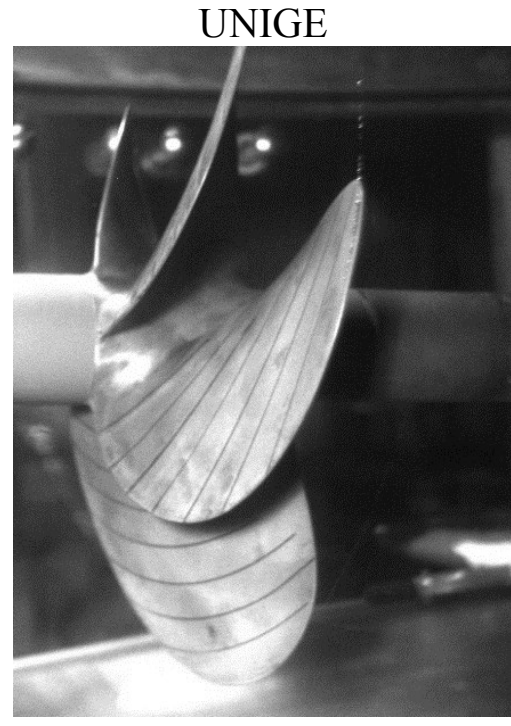

$5^{\circ}$ inclination angle

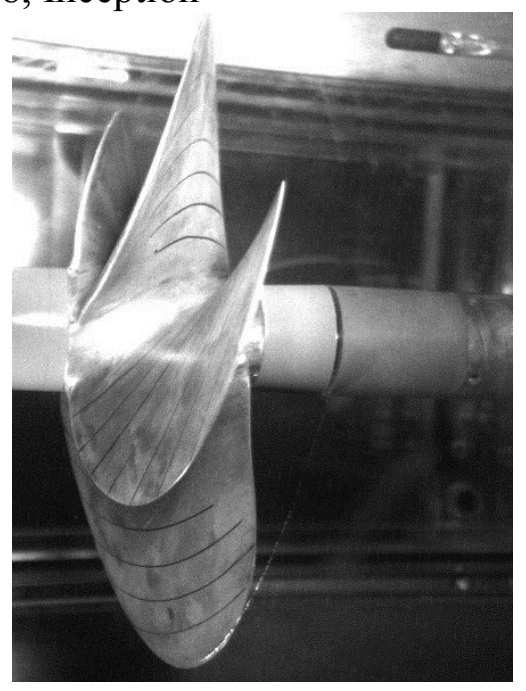

$\mathrm{C} 4: \mathrm{K}_{\mathrm{T}}=0,19, \mathrm{~J}=0.5, \sigma_{\mathrm{n}}=3.48,0^{\circ}$ inclination angle 


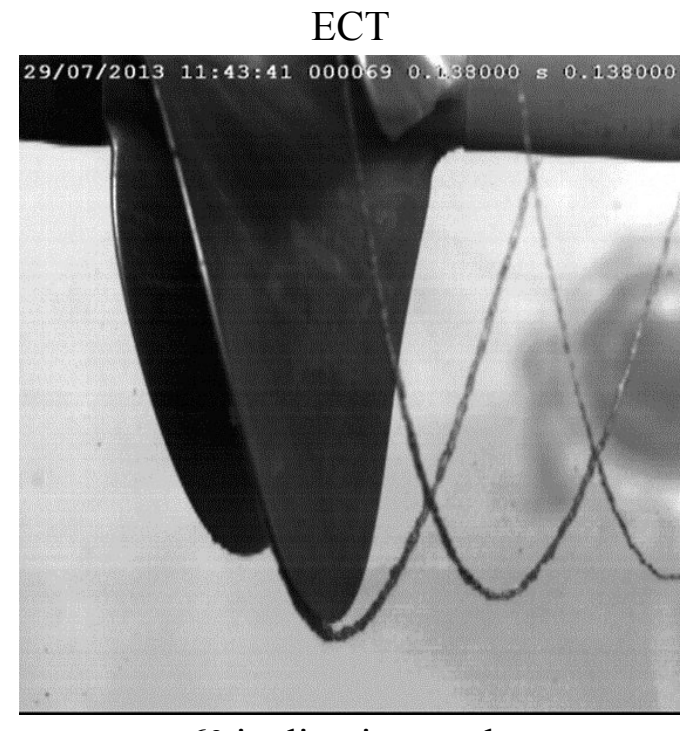

$6^{\circ}$ inclination angle

C5: $\mathrm{KT}=0,19, \mathrm{~J}=0.5, \sigma \mathrm{n}=2.03$

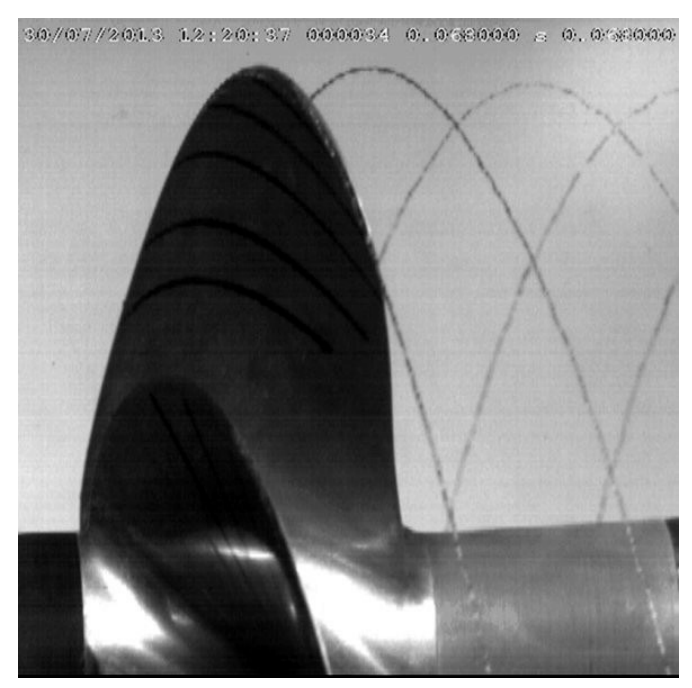

$\mathrm{C} 5: \mathrm{KT}=0,19, \mathrm{~J}=0.5, \sigma \mathrm{n}=2.03,0^{\circ}$ inclination angle

UNIGE

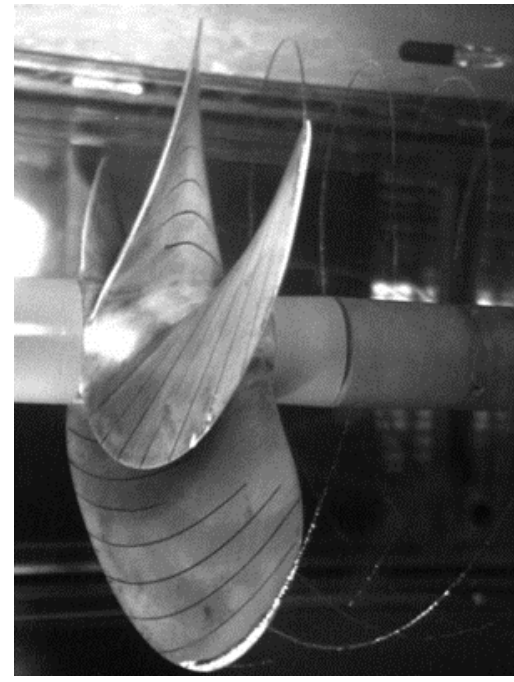

$5^{\circ}$ inclination angle

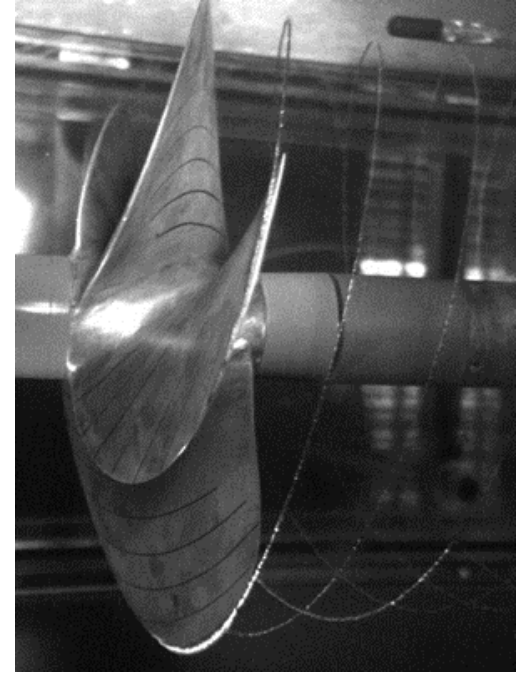




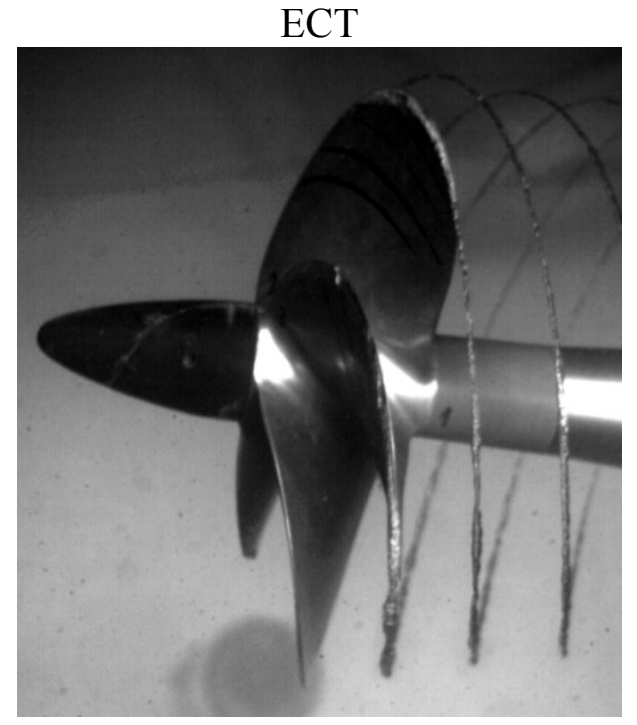

$6^{\circ}$ inclination angle C6: $\mathrm{K}_{\mathrm{T}}=0,19, \mathrm{~J}=0.5, \sigma_{\mathrm{n}}=1.13$

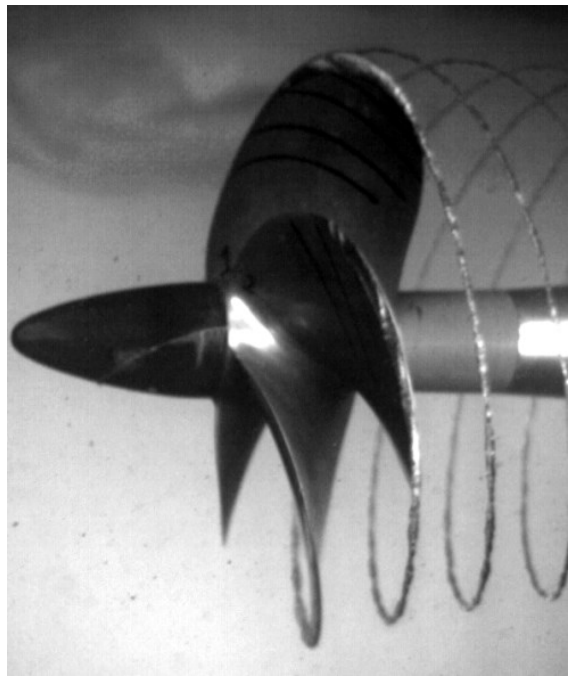

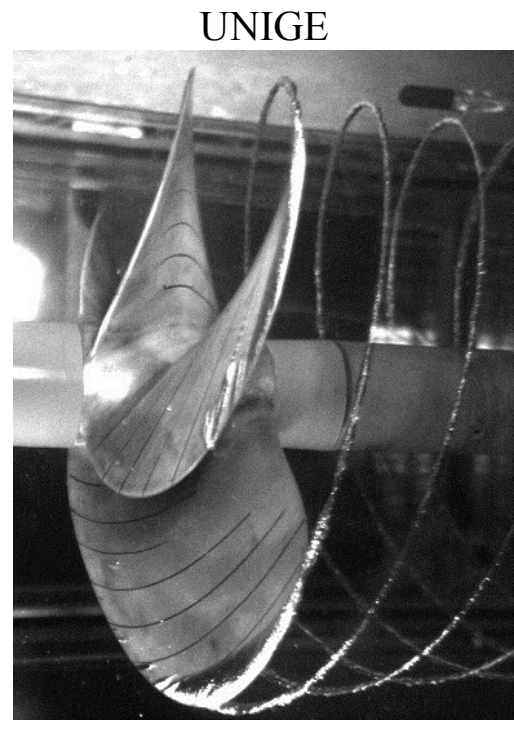

$5^{\circ}$ inclination angle

C6: $\mathrm{K}_{\mathrm{T}}=0,19, \mathrm{~J}=0.5, \sigma_{\mathrm{n}}=1.13,0^{\circ}$ inclination angle 


\section{Noise measurements}

In this section, measuring and post-processing procedures adopted for radiated noise are described. These procedures are in large part similar for the two facilities, thus a general description is given for both facilities and differences or special techniques adopted are described only when deemed necessary.

A common practice in the analysis and presentation of the noise levels is to reduce the measured values of Sound Pressure Levels (SPL) in each 1/3 Octave band to an equivalent $1 \mathrm{~Hz}$ bandwidth by means of the correction formula recommended by ITTC using Equation 6 .

$$
\mathrm{SPL}_{1}=\mathrm{SPL}_{m}-10 \log \Delta f
$$

Equation 6

where $\mathrm{SPL}_{1}$ is the reduced sound pressure level to $1 \mathrm{~Hz}$ bandwidth in $\mathrm{dB}$; re $1 \mu \mathrm{Pa}, \mathrm{SPL}_{\mathrm{m}}$ is the measured sound pressure level at each centre frequency in $\mathrm{dB}$; re $1 \mu \mathrm{Pa}$ and $\Delta \mathrm{f}$ is the bandwidth for each $1 / 3$ Octave band filter in $\mathrm{Hz}$.

The corrections for the tunnel background noise measurements are applied to the measured total noise levels in order to determine the net cavitation noise. The background noise measurements are made without the model propeller which is replaced by a dummy hub (Bertschneider et al., 2014). The background noise correction is applied depending on the level of the difference following the procedure in ANSI/ASA S12.64-2009/Part1. When the difference is smaller than $3 \mathrm{~dB}$, the result is discarded. In case of a difference between 3 and $10 \mathrm{~dB}$, the results are corrected according to Equation 7 and no correction is applied in case of the difference being greater than $10 \mathrm{~dB}$. The regions where the data is discarded due to low signal to noise ratio (i.e. less than $3 \mathrm{~dB}$ ) are also omitted from the figures 16 to 37 . Thus, there exists some regions with lack of data within the figures.

$$
\left.\left.S P L_{N}=10 \log \left[10^{\left(S P L_{T} / 10\right.}\right)-10^{\left(S P L_{B} / 10\right.}\right)\right]
$$

where subscripts $N, T$ and $B$ indicate net, total and background respectively.

The ITTC also required that the sound pressure levels are corrected to a standard measuring distance of $1 \mathrm{~m}$ using the following relationship. 


$$
\mathrm{SPL}=\mathrm{SPL}_{1}+20 \log (r)
$$

Equation 8

Where; SPL is the equivalent $1 \mathrm{~Hz}$ at $1 \mathrm{~m}$ distance sound pressure level (in $\mathrm{dB}$; re $1 \mu \mathrm{Pa}$ ) and $r$ is the distance between the acoustic centre of the noise source (centre of the propeller disc) and hydrophone. The dynamometer position and reference distance (hydrophone distance) for each inclination angle are kept 0.61 meter at ECT by adjusting the height of the dynamometer.

Net noise levels are scaled to the reference distance in a different way at the UNIGE cavitation tunnel. Actually, test sections transfer functions for the three hydrophones have been measured exploiting an underwater electronic transducer with known emitting characteristic. Transfer functions are measured in the frequency range 100:100000 Hz, however results below $300 \mathrm{~Hz}$ are less reliable because of uncertainties in the measurement of the transducer transmitting voltage response in free field. The procedure adopted is described more in details in (Tani et al., 2015a, 2015b).

Model scale results are presented in terms of levels of the non-dimensional pressure coefficient $\mathrm{K}_{\mathrm{P}}$, defined as follows:

$$
K_{P}=\frac{P}{\rho N^{2} D^{2}} \quad \text { and } \quad L_{K P}=20 \log _{10} \frac{K_{P}}{10^{-6}}
$$

Equation 9

where $L_{K P}$ indicates the pressure coefficient level in $\mathrm{dB}$ by using a reference value of $10^{-6}$.

According to the aims of the round robin campaign, a post-processing procedure has to be defined in order to compare results from different facilities taking into account all differences, as far as possible. For present work it was decided to perform extrapolate results to full scale reference conditions. More details on this extrapolation are given in section 6.5 where it is applied. Otherwise results are presented in terms of levels of non-dimensional pressure coefficient.

As already mentioned tests at ECT have been carried out prior to the beginning of the round robin campaign and include a significant number of operational conditions and shaft inclination configurations, as presented by (Aktas et al., 2015). On the other hand, tests at UNIGE included 
different propeller revolution rates, oxygen contents and small variations of the functioning parameters. Consequently, all these tests together constitute a wide investigation of the effects that different propeller / cavitation tunnel parameters have on cavitation noise. All these investigations provide important data for the aims of the round robin campaign, allowing estimation of how different procedures and facilities operational conditions may influence results. However, it has to be remarked that results of these analyses describe more consistently the propeller noise measurements for the facilities where they are carried out, while they could lose generality with respect to other facilities.

As an example the influence of gas content on cavitation tests strongly depends on the characteristics of the facility, especially for what concerns general water treatment and nuclei control devices adopted. The following subparagraphs present results subdivided in order to analyse each aspect separately and providing relevant examples. Only the analyses relative to UNIGE tests are reported here since results of the whole campaign carried out by ECT have been already presented in Aktas et al (2015). In the last paragraph, according to aims of the round robin tests, some direct comparisons between the ECT and UNIGE test results are reported.

\subsection{Influence of hydrophones position and transfer functions (UNIGE)}

The three hydrophones adopted at UNIGE are characterized by rather different positions, as shown in Figure 4. In particular, two of them (H2 and $\mathrm{H} 3)$ are placed directly inside the tunnel test section. These configurations may generally suffer high background noise levels because of the self-noise due to the tunnel flow on the sensors. This problem was not severe during the current tests thanks to the relatively low flow speed values adopted (maximum about $4 \mathrm{~m} / \mathrm{s}$ ). However, due to the dimensions of the test section these hydrophones are always rather close to the propeller, thus measurement from these sensors may be partially affected by near field effects.

The third hydrophone (H1) is placed outside the test section, in a small PMMA thank filled with water and placed on the bottom observation window. This particular configuration allows to enhance, at least partially, the signal to noise ratio by reducing the hydrophone self-noise. On the other hand, noise propagation towards the external tank is less effective at medium-low frequencies, as clearly shown by the relative transfer functions, see Figure 16. Actually, as it can be observed, the transfer function of $\mathrm{H} 1$ hydrophone, below $3 \mathrm{kHz}$, is $10 \mathrm{~dB}$ on average 
lower than those of the other two sensors. Finally, the distance from the propeller in this case is increased, thus near field effects are reduced.

Even if the external configuration is the one for which the effects of noise propagation are more evident, the measurements from all the three sensors are affected by the confined environment effect. This effect is taken into account by means of transfer functions measured for each hydrophone configuration. For this purpose, the ITC1001 underwater transducer has been adopted. Transfer function measurements are carried out also considering different positions of the noise sources (the electronic transducer). These positions are usually chosen around the ones where larger cavitation is present. Then the final transfer functions, shown in Figure 16, are defined as the averages. In present case, the source has been placed in correspondence to propeller centre disk and at a radial position corresponding to $r / R=0.5$. This distance from the centre was chosen because of the dimensions of the transducer which has a diameter of about $100 \mathrm{~mm}$, thus comparable with those of the model propeller blades. With this radial position twelve angular positions were considered, namely from $0^{\circ}$ to $360^{\circ}$ with a spacing of $30^{\circ}$.

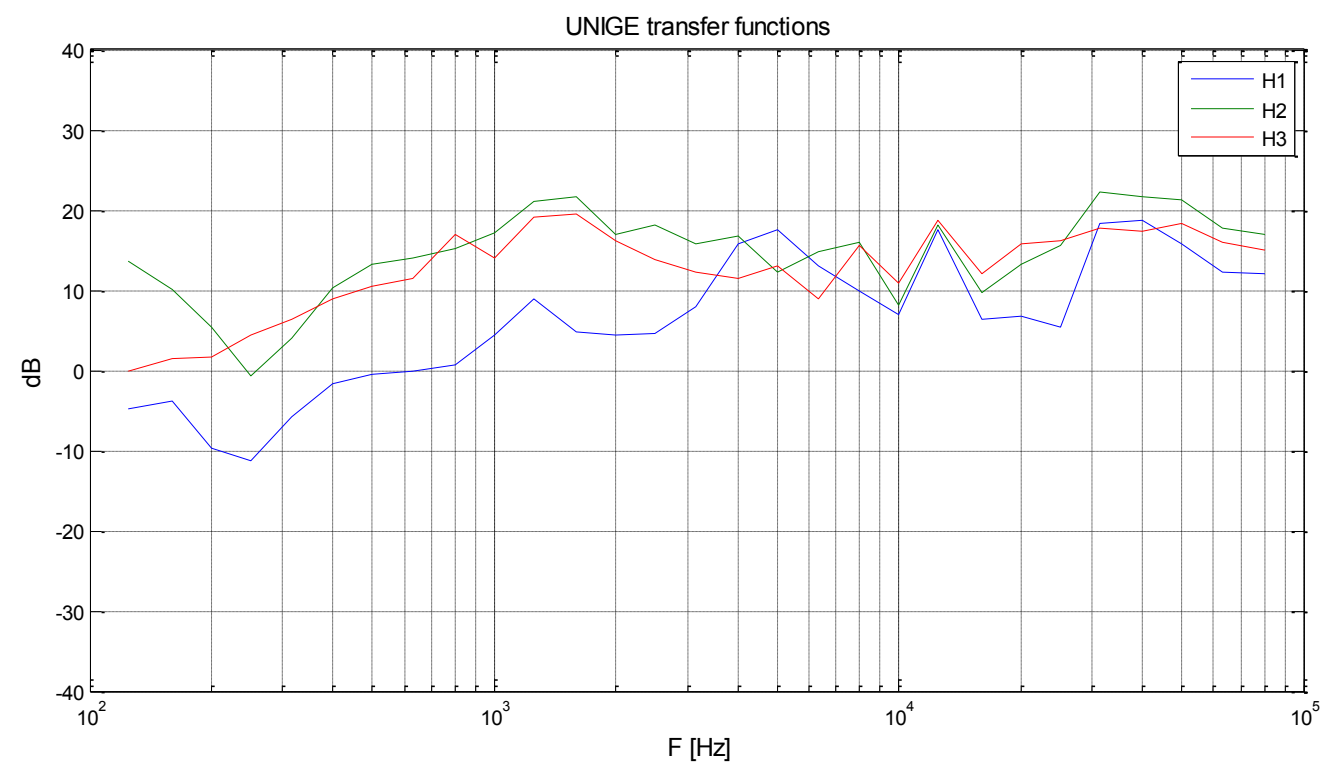

Figure 16: UNIGE cavitation tunnel transfer functions.

In order to show a first example of noise measurements at UNIGE and to analyse the effects of hydrophone positions and transfer functions corrections noise spectra measured for condition $\mathrm{C} 1$ are reported in Figure 17 and Figure 18, without and with application of the transfer functions, respectively. For the sake of clarity, 1/3 Octave band representation is adopted. Spectra shown in these figures have been corrected for background noise. 


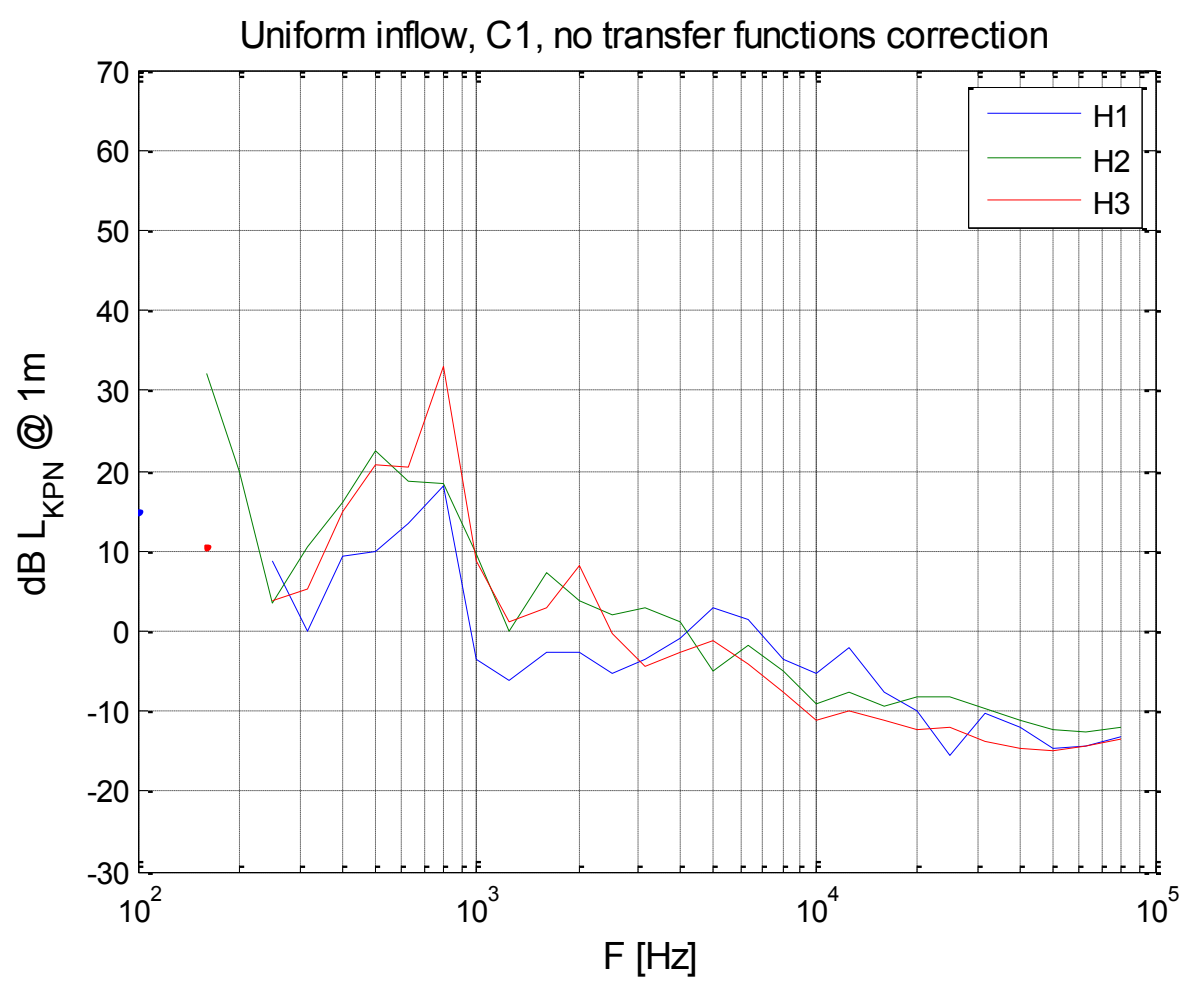

Figure 17: Comparison between hydrophones spectra without transfer function correction.

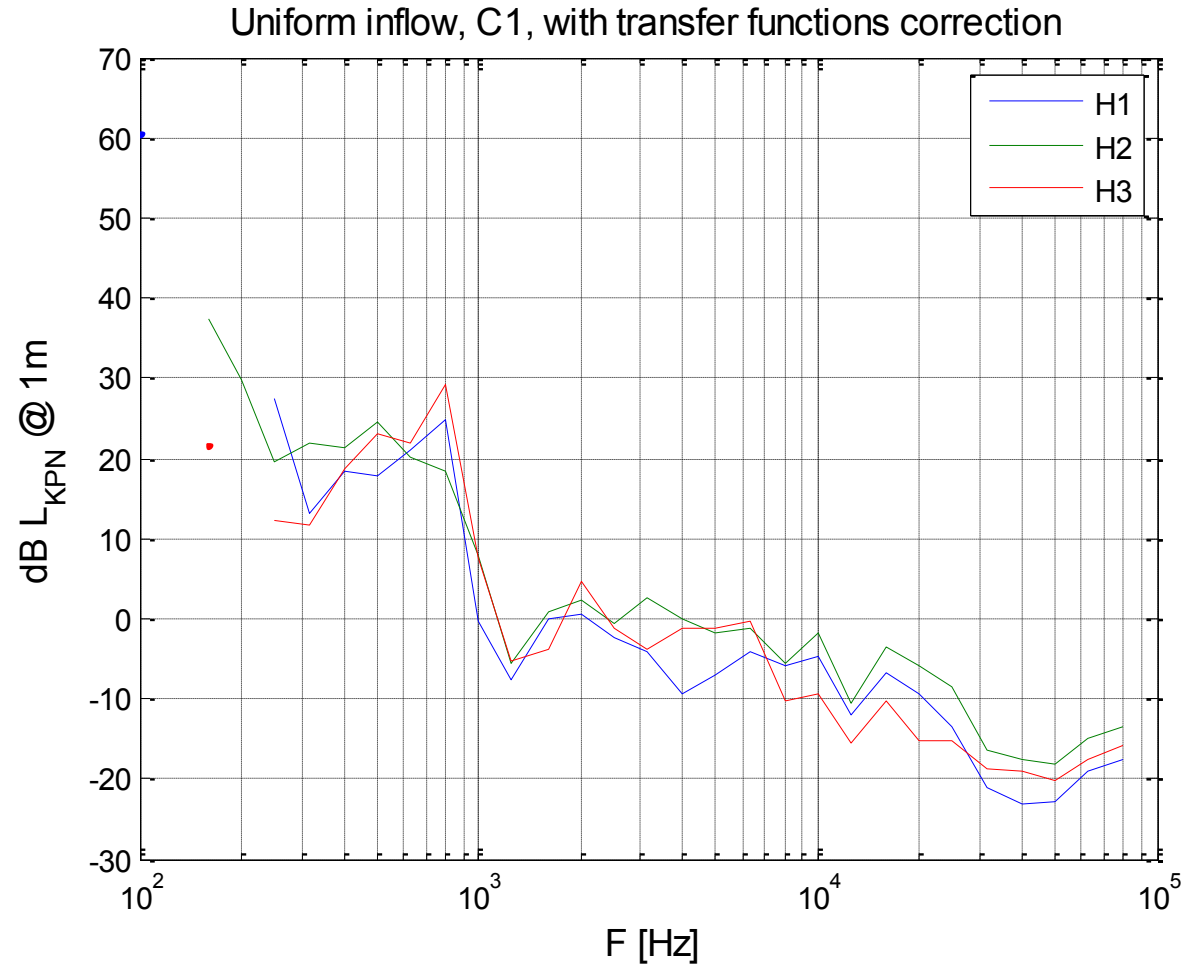

Figure 18: Comparison between hydrophones spectra with transfer function correction. 
As it can be seen, the three measured spectra, scaled to the reference distance according to the $20 \log (\mathrm{R})$ (without application of transfer functions), present considerable differences, as shown in Figure 17. Thus these differences cannot be ascribed only to the different distances of the hydrophones, especially if the spectrum from the external hydrophone (H1) is considered. Applying transfer functions, differences between spectra are significantly reduced as shown in Figure 18, even if some discrepancies are still present, and the shapes of the three spectra become similar.

This allows to compute the final noise prediction as the average of the spectra measured by the three hydrophones, however for the following analyses it is preferred to consider only one sensor, without performing averages. For this purpose, hydrophone $\mathrm{H} 3$ will be considered for the various sensitivity analyses, being the one for which more measurements are available. However, from the point of view of the most reliable noise prediction, hydrophone $\mathrm{H} 1$ should be considered since it should be the less affected by the near field effect, as previously explained.

For what regards the performances of the sensors in terms of signal to noise ratio it can be noticed that this is higher than $3 \mathrm{~dB}$ for a rather wide range of frequencies for all the three hydrophones. In addition, Figure 19 and Figure 20 confirm that, thanks to the low water speed in the tunnel, hydrophones self-noise is not problematic; as it can be seen in the same figures, frequency bands for which the net noise is perceivable are slightly more in the case of the hydrophones H2, inside the tunnel flow, with measured values also at lower frequencies. From the point of view of detectability of cavitation noise, it has to be remarked that net noise levels have been reported, which means that the background noise correction has been already applied. The result of this correction should in principle represent propeller noise, purified from unwanted background noise disturbances.

Anyway it could be useful to analyse the problem of background noise more in detail. For the present campaign the background noise has been measured reproducing tunnel operational conditions with the dummy hub in place of the propeller. Thus, the net noise levels computed and here presented may be caused not only by cavitation but also by any noise source connected with the operation of the propeller inside the tunnel. This includes the noise of the driveline, which may be higher under propeller load, and propeller singing. A possible way to better identify cavitation noise is to define the background noise as the maximum envelope of noise measured with the non cavitating propeller (at high static pressure) and noise measured at 
current static pressure with the dummy hub. Unfortunately, it was not possible to use this procedure, sometimes adopted at UNIGE, since for the current operational conditions the propeller also cavitates at atmospheric pressure and the tunnel pressure cannot be increased over the atmospheric level.

To get a deeper insight into the problem, it could be worth analysing narrowband spectra since this may allow identifying some possible unwanted noise components. Only two examples are reported in Figure 19 and in Figure 20 to clarify these aspects, the complete analysis is not included in present work for the sake of limited space. However, some of its conclusions may be utilized for a better interpretation of noise results for all operational conditions considered in this work.

As an example, an anomalous peak is often present around $800 \mathrm{~Hz}$, as shown in Figure 19 and Figure 20. This noise component is probably due to the vibration of one of the foils housing the hydrophones inside the tunnel.

Unfortunately, it coincides partially with the spectral hump due to the tip vortex, which is however well visible and identified in the spectra reported for condition $\mathrm{C} 1$ between $300 \mathrm{~Hz}$ and $1000 \mathrm{~Hz}$. In most cases cavitation noise overcomes all the background noise due to foil vibration except its main tone. 


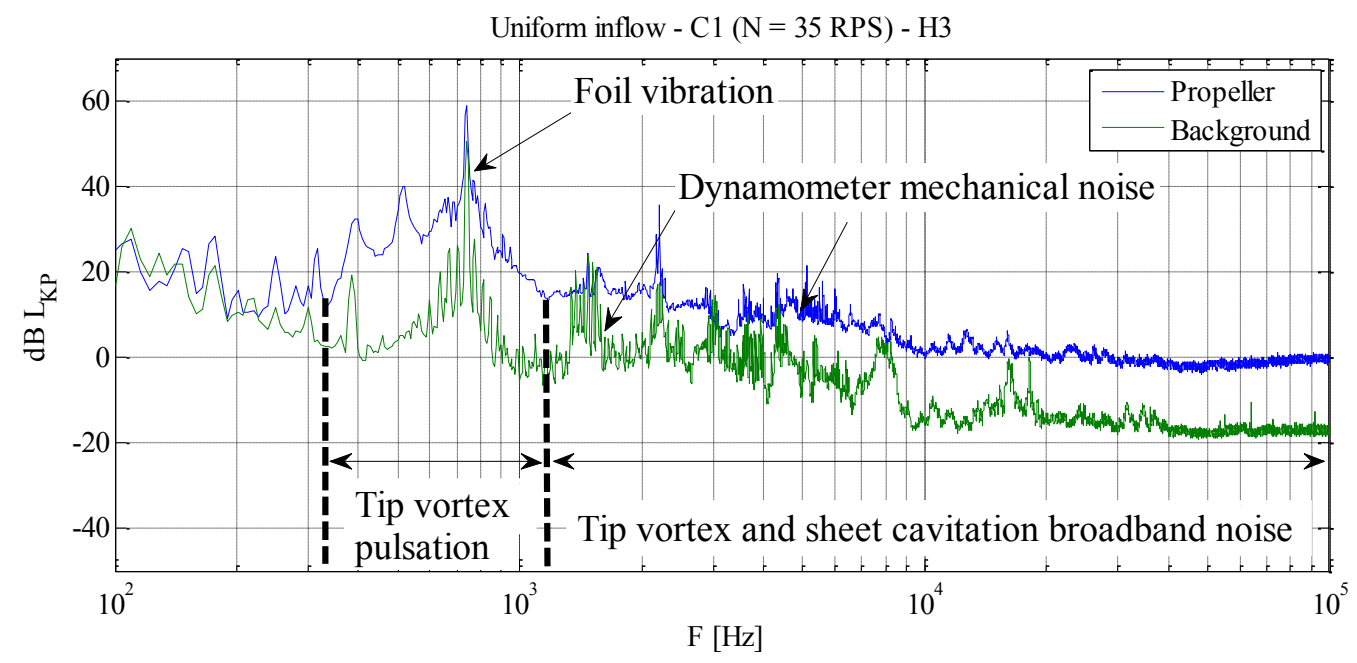

Figure 19: Narrowband analysis, propeller noise compared with background noise with relevant cavitation.

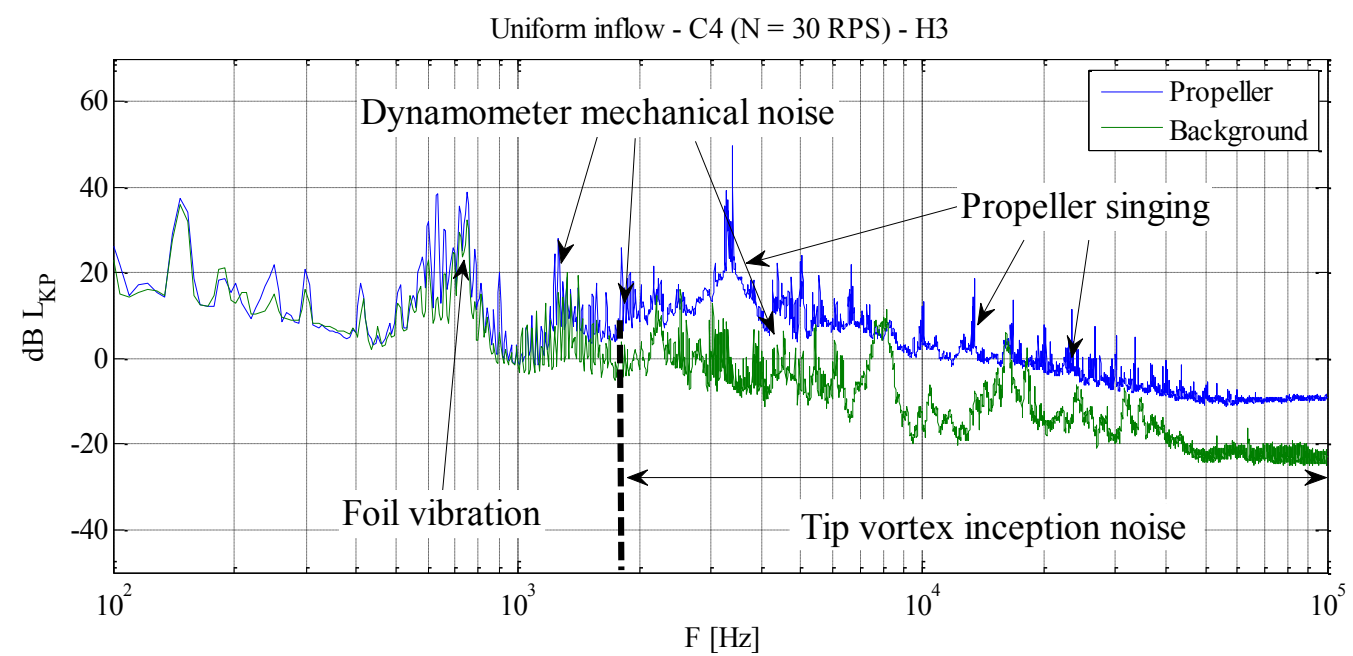

Figure 20: Narrowband analysis, propeller noise compared with background noise with limited cavitation and relevant propeller singing.

Other tonal components are also present between $6000 \mathrm{~Hz}$ and $12000 \mathrm{~Hz}$. The characteristic frequencies of such components are multiple of the shaft rate. These tones are likely to be generated by the dynamometer driveline and are higher when the propeller is present instead of the dummy hub because of the higher load acting on the driveline. However, analysing the narrowband spectra, the effect of cavitation is pointed out by the increased levels of the continuous part of the spectra. As a consequence, net noise levels computed are still representative of cavitation noise even if they could be partially altered by the presence of dynamometer tonal noise. 
From the point of view of cavitation noise, it is not easy to consistently identify different components in the spectra and assess which is the underlying generation mechanism.

Some indications of the noise contribution associated to the main cavitating phenomena are available in literature, as for example in (Carlton, 2007).

Generally, all cavitation phenomena may contribute to both tonal noise and broadband noise. In particular, the contribution to tonal noise is more important where large volume variations occur because of periodically variant inflow conditions on propeller blades. An effective example of this noise generation mechanism is represented by suction side sheet cavitation occurring in the wake peak of a single screw ship. Anyway also other cavitation typologies may contribute to tonal noise for the same reasons.

On the other hand, cavitation is always characterized by a certain amount of random phenomena which contribute to the continuous part of the spectrum.

Generally, the continuous spectrum of cavitation noise features a flat shape with high levels also at high frequencies when cavitation presents impulsive phenomena, such as in the case of vortex bursts and cavities collapses.

Accordingly, wide band spectra are usually observed when bubble or cloud cavitation are present, or when the inflow characteristics produce sudden bursts and collapses of vortices and sheet cavitation.

Thus, also the more stable suction side sheet cavitation may produce broadband noise, by means of unsteady or impulsive phenomena like the detachment of cavitation sheet into a more cloudy cavitation and the collapse of shed cavities (Konno et al., 2002).

Pressure side cavitation usually produces high noise levels at medium high frequency band, since in this case, cavities dynamics are generally more violent than in the case of suction side sheet cavitation or tip vortex cavitation. Actually, when cavitation occurs on the blade face, cavities are generated in a rather limited low pressure area and collapse phenomena occur, as soon as they exit this area. This behaviour is evidenced, for example, by the limited persistence of pressure side vortices downstream propeller blades.

Tip vortex spectrum is a particular case since it is characterized by a medium-low frequency hump in the spectrum. This hump is associated to pressure fluctuations generated by the vortex, whose volume is characterised by pulsations around its natural frequencies, as described for example in (Pennings et al., 2015a, 2015b).. 
In addition to this hump, the vortex spectrum features also a wide band high frequency part since in parallel to the above mentioned pulsation, random phenomena may be present, more or less important depending on propeller inflow characteristics. Usually tip vortex contribution at high frequency is significant only if bursts and collapses occur, otherwise it should be lower than noise produced by other phenomena as those previously mentioned.

Keeping in mind these general rules, reported spectra are in good agreement with observed cavitation pattern. Condition $\mathrm{C} 1$ is characterized by the presence of significant tip vortex and sheet cavitation while in correspondence to condition $\mathrm{C} 4$ only a thin tip vortex near inception is present. Accordingly, the spectrum measured for condition $\mathrm{C} 1$ features a hump between 400 $\mathrm{Hz}$ and $1000 \mathrm{~Hz}$, as shown in Figure 19 narrowband representation and in Figure 17 and Figure 18 adopting one third octave band spectra.

At higher frequencies the continuous part of the spectrum measured with the propeller is higher than background noise by about $10 \mathrm{~dB}$, on average. Levels slightly decay moving to higher frequencies. This part of the spectrum is representative of the broadband noise generated by both tip vortex and suction side cavitation by means of the mechanisms just described.

This phenomenology is present, with different extensions, for the major part of considered operational conditions which, consistently, are characterized by qualitatively similar noise spectra. One important exception is condition C4. In this case, cavitation noise spectrum is visible only for a small portion at high frequency, because of the relatively low radiated levels, if compared to background noise. Nevertheless, the characteristics of this spectrum are in good agreement with the presence of tip vortex at inception, whose intermittent and unstable nature, combined with the reduced dimensions of cavities, usually produces similar broadband spectra at rather high frequency.

Finally, regarding condition C4, it is worth analysing the presence of a significant tonal at 3400 , plus numerous multiples at higher frequencies. These tones, easily audible, represent a case of propeller singing. This phenomenon has been encountered mainly for condition C4 at 30 RPS, while in other cases it was not present or rather weak. It has to be remarked that it appears to interact with cavitation and in this particular case also broadband noise is influenced. 


\subsection{Influence of the oxygen content}

As already mentioned, the operational conditions considered for the round robin campaign cover the atmospheric and high vacuum conditions, as well as the conditions near to the cavitation inception and extreme cavitation. Due to this, the optimal setup for each condition may be rather different, especially for what regards the water quality, thus it was decided to perform the tests at UNIGE cavitation tunnel considering different oxygen content levels to better fit with the actual operational conditions.

In principle, the oxygen content during tests should be equal to the saturation value at the operating static pressure. However, as explained in the inception paragraph, the UNIGE cavitation tunnel suffers of a difficulty in eliminating free bubbles due of its limited height. Consequently, oxygen content levels adopted for tests are always a trade off between theoretical values and previous experiences; finally, the correct level is selected after some preliminary tests. For present campaign, three oxygen levels have been considered:

- $\quad \mathrm{DO} 2=6.2 \mathrm{ppm}(79 \%$ of saturation at atmospheric pressure $)$

- $\quad \mathrm{DO} 2=5 \mathrm{ppm}(55 \%$ of saturation at atmospheric pressure $)$

- $\quad$ DO2 $=3.7 \mathrm{ppm}(41 \%$ of saturation at atmospheric pressure $)$

These levels represent typical high, medium and low levels of oxygen content according to UNIGE cavitation tunnel standards.

In addition, considering the aims of the round robin tests, it was decided to perform a sort of sensitivity analysis of the radiated noise levels to the oxygen content applied, thus all the operational conditions have been tested with all the three oxygen levels (with only few exceptions).

A selection of the more representative results is presented for the effect of the oxygen content. Actually, observed behaviours can be subdivided in three groups corresponding to: near to inception conditions; moderate cavitation condition; and extreme cavitation condition. Examples for these categories are shown in Figure 21, Figure 22 and Figure 23 for the configuration of level shaft while the same trends have been observed also for the inclined shaft configuration. 
The general comment for the effect of the oxygen content is that the noise spectrum for each level of oxygen content is rather similar, confirming the good repeatability of the results and evidence of a limited influence of the water quality on the noise measurements of the present campaign. This limited effect is also a consequence of the fact that the considered oxygen levels, even if they are different, represent typical values for tunnel activities.

Moreover, passing to a deeper analysis, some effects are visible in the case of the near to inception condition (C4), as shown in Figure 21. In fact, higher levels of sound pressure levels can be observed with the higher oxygen content. This is in good agreement with the effect of cavitation nuclei which influences the inception point, as largely demonstrated by (Korkut and Atlar, 2002), and making cavitation more stable.

The above highlighted trend is appreciable above $8 \mathrm{kHz}$, where cavitation noise starts to overcome the background noise as already discussed, and this is in good agreement with typical spectra of noise radiated by cavitation at inception (Kuiper, 2012).

The trend is less clear moving to lower frequencies, however for those frequencies cavitation noise is likely to be masked, at least partially, by other noise sources; actually, for this condition, propeller singing occasionally occurred. As an example, the two peaks at 3 and $5 \mathrm{kHz}$ are related to the presence of remarkable tonal component due to propeller singing.

For what regards as the moderate conditions, condition $\mathrm{C} 1$ is presented in Figure 22 but also C2, C5 and partially C6 can be belonged to this group. In such cases differences between spectra are perfectly negligible and the oxygen content seems to have almost no effect. This is reasonable due to the fact that for such conditions cavitation is well developed and not intermittent, while the amount of free bubble in the tunnel is acceptable, without absorption and scattering phenomena.

Such phenomena are instead easily detectable in the spectra measured for condition $\mathrm{C} 3$, especially above $4 \div 5 \mathrm{kHz}$, as shown in Figure 23 . Actually, because of the combined effects of extreme cavitation occurring on the propeller, and high vacuum level, the amount of free bubbles in the tunnel becomes relevant and continuous growth takes place. Reducing the oxygen content the amount of free bubbles decreases and accordingly also their dampening effect on noise, as clearly shown by reported spectra, even if available only for two oxygen levels. 


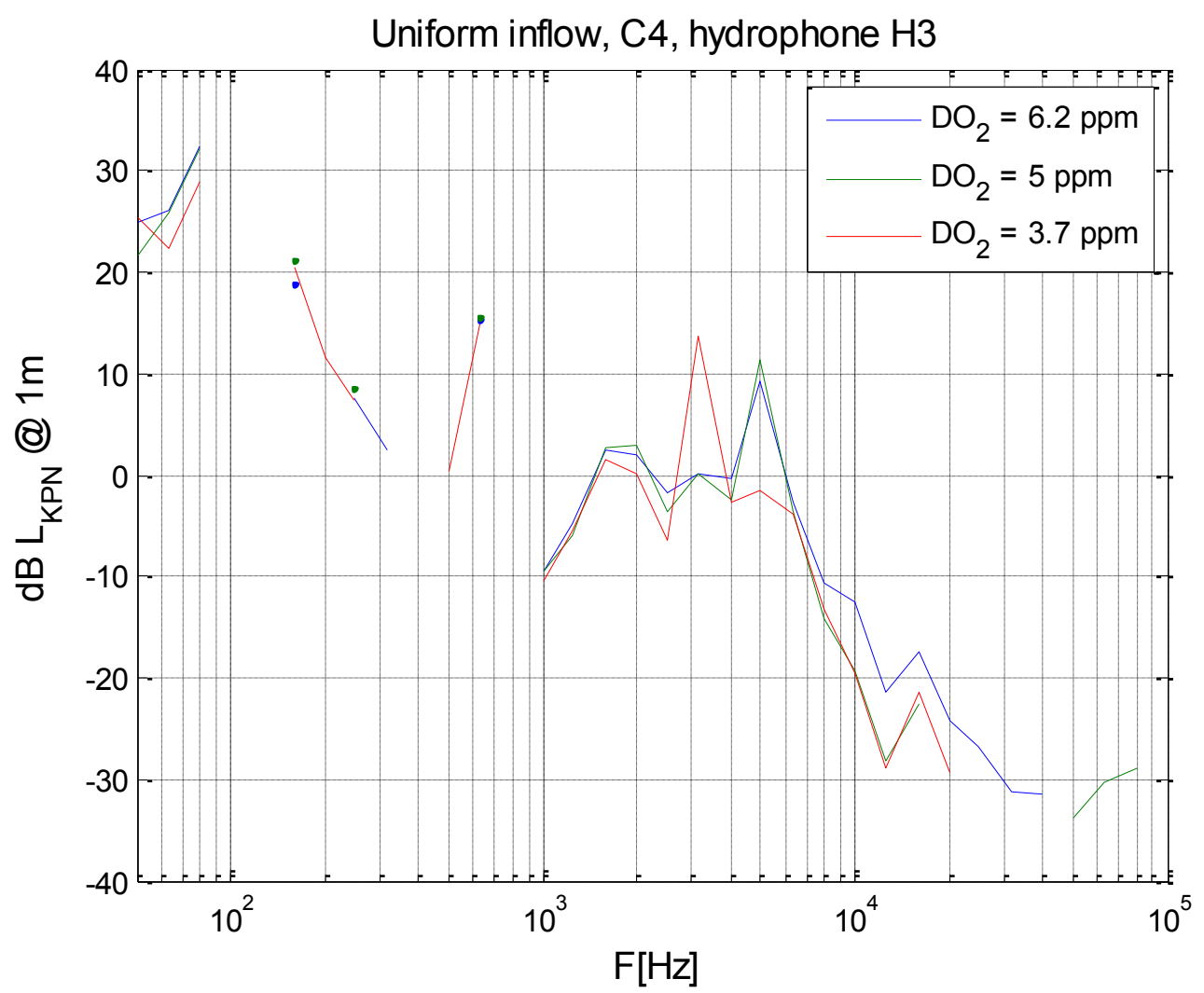

Figure 21: Influence of the oxygen content on radiated noise, near to inception condition.

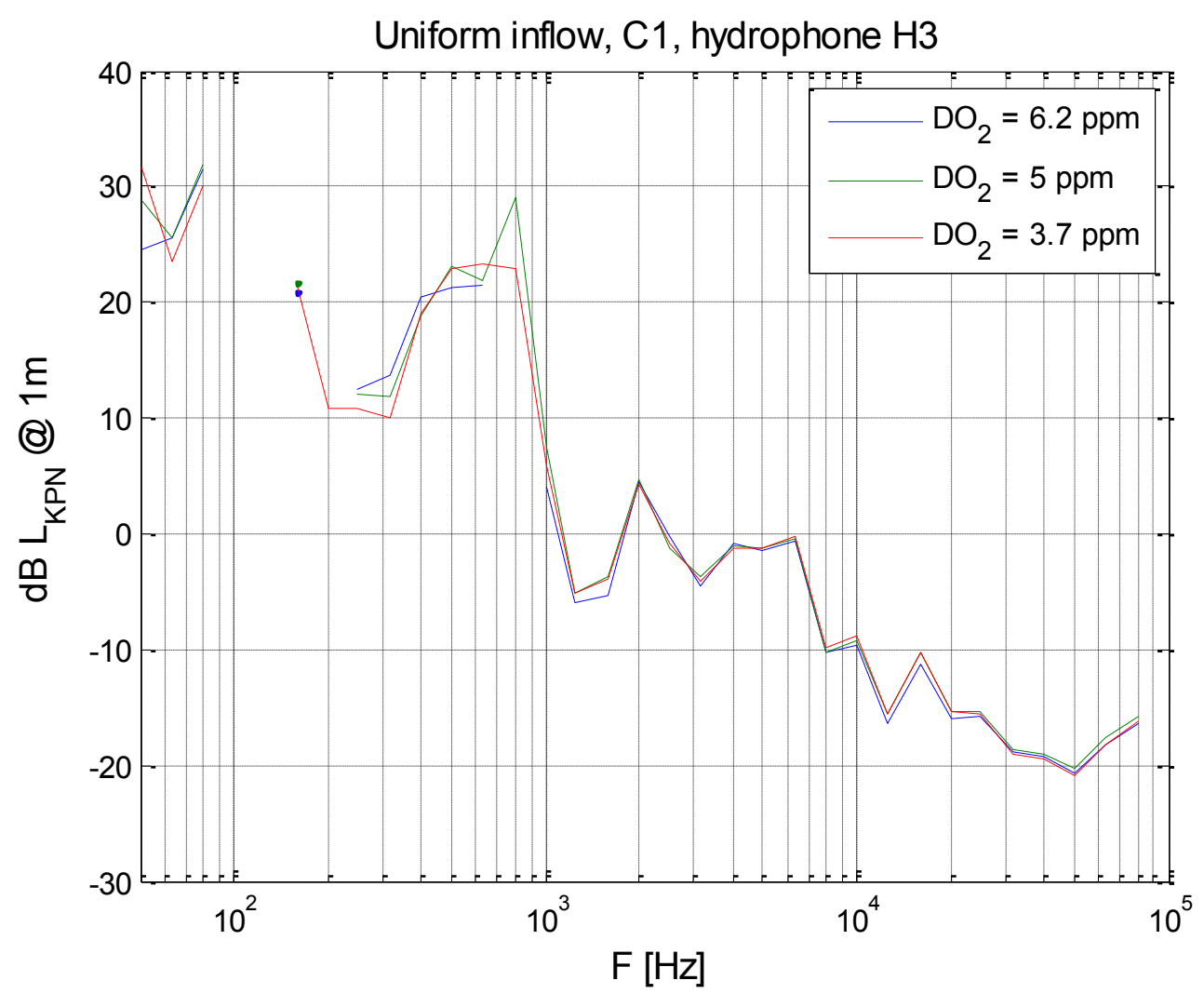

Figure 22: Influence of the oxygen content on radiated noise, intermediate condition. 


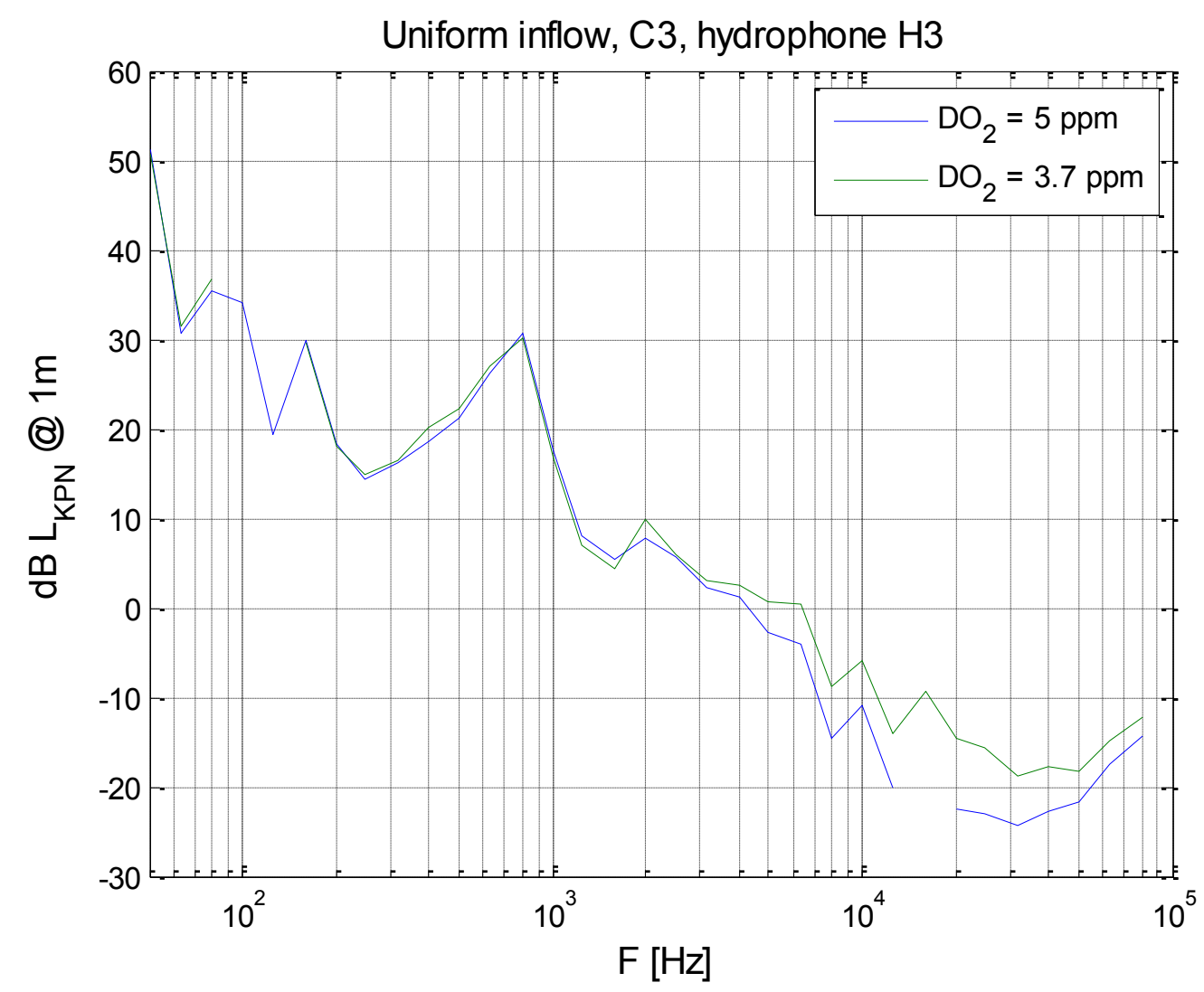

Figure 23: Influence of the oxygen content on radiated noise, large cavitation extension.

\subsection{Influence of the shaft revolution rate}

The same approach considered for the water quality has been applied also to the influence of the shaft revolution rate on propeller noise. The importance of this aspect for the round robin campaign is well exemplified by this work. Actually, as explained in paragraph 2.4, it was not possible to replicate at the UNIGE cavitation tunnel the same operational conditions considered as ECT but it was necessary to reduce the propeller revolution rate and the flow speed accordingly. Similar issues may occur also at the other facilities involved in the campaign thus it was deemed of some interest to study, even if considering only two values, this aspect.

During preliminary tests different revolutions rates were considered, from 20 RPS to 35 RPS, in order to investigate which condition allowed achieving the maximum signal to noise ratio, without, on the other hand, resulting in too low static pressure inside the tunnel, with consequent problems related to free bubbles. According to results of these tests and usual procedures adopted, tests were performed keeping propeller revolution as high as possible, thus 35 RPS for the majority of tests and 30 RPS as second values for this investigation. 
Analogously with the previous paragraph, the most representative results are reported in Figure 24, Figure 26 and Figure 27. In addition, in these graphs frequencies are normalized with respect to the blade passage frequency (BPF), an example of comparison without normalizing the frequency is reported in Figure 25.

Also in this case three main different behaviours can be identified, corresponding to the same functioning conditions considered for the oxygen content. The first, more common situation is represented by $\mathrm{C} 1$ as one of the intermediate conditions and shown in Figure 24. In such cases, spectra in terms of $K_{P}$ coefficient are very similar, confirming that, as far as model scale is concerned, noise levels scale with the square of propeller revolution rate. This behaviour is largely confirmed also by the other functioning conditions but with some exceptions for conditions $\mathrm{C} 3$ and $\mathrm{C} 4$.

In particular, for condition $\mathrm{C} 3$ the difference between spectra increases above $10 \mathrm{BPF}$, as shown in Figure 26. At medium-high frequencies the dampening effect of free bubble becomes relevant and this may partially explain observed differences. Actually, the tunnel pressure is slightly higher with the higher revolution rate and this increases the amount of bubbles destroyed during the passage in the lower duct of the tunnel. At lower frequencies, differences are almost negligible, as observed also for the effect of oxygen content. This may suggest that noise absorption problems affect these measurements only at medium-high frequencies, however further verifications are needed to confirm this hypothesis.

Finally, remarkable differences are observed in the case of the near to inception condition $\mathrm{C} 4$, as shown in Figure 27; however, in this case the differences are not only due to the dynamics of the tip vortex inception and related noise. Propeller revolution rate is expected to influence the tip vortex inception because of the increased Reynolds number and accordingly this higher levels should be measured with higher revolution rates. However, the differences in Reynolds number are limited and the effects of the anticipated inception on the radiated noise are expected to be negligible, or, at least, very limited.

Contrary to the above trend, the noise levels are significantly higher in the case of the lower rotational speed. The reason for this is that the singing phenomenon, observed also at 35 RPS, is significantly stronger at 30 RPS. From this point of view, it has to be remarked that for this propeller model, singing did not occur as a single tone in the spectrum, on the contrary its occurrence, easily detectable even by ear, coincides with the appearance in the spectrum of a 
large number of tones together with an increase of broadband levels, as previously shown. It is believed that this phenomenon is due to a particular interaction between structural singing of the propeller and cavitation. Actually, singing varies significantly, and finally disappears modifying the static pressure in the tunnel. A complete characterization of this phenomenon is beyond the purposes of this paper and of the round robin campaign as well, however it is important to keep it in mind for a consistent interpretation of the measured noise results.

For what regards the frequency, it is expected to scale differently depending on the driving noise mechanism. Actually, frequencies of propeller hydrodynamic noise should be proportional to the revolution rate. On the other hand, frequencies of cavitation noise should scale with the inverse of the time scale of bubble pulsation or collapse, as discussed as an example in (Bark, 1985; Blake, 1986, 1984). This leads, according to classical bubble dynamics, to Equation 10:

$$
\frac{f_{1}}{f_{2}}=\frac{n_{1}}{n_{2}} \sqrt{\frac{\sigma_{1}}{\sigma_{2}}}
$$

This means that, considering points with same cavitation number the proportionality to the revolution rate should hold also for cavitation noise. However, this is only partially confirmed by spectra measured in present campaign, even if it could be pointed out that the two propeller revolution rates considered are too much close each other to allow appreciating frequency scaling.

Nevertheless, a certain trend is visible for conditions C1 (and other not reported intermediate condition) and C3. Actually, the hump in the spectrum reasonably identified as tip vortex noise seems to scale in good accordance with the revolution rate. On the contrary, medium-high frequency broadband noise seemed to be characterized by a weaker dependence on the revolution rate, actually these parts of the spectra seem more similar each other without normalizing the frequency, as visible in Figure 25. The same figure confirms that, without frequency normalization, the humps present below $1 \mathrm{kHz}$ are slightly shifted, according to what stated above. 


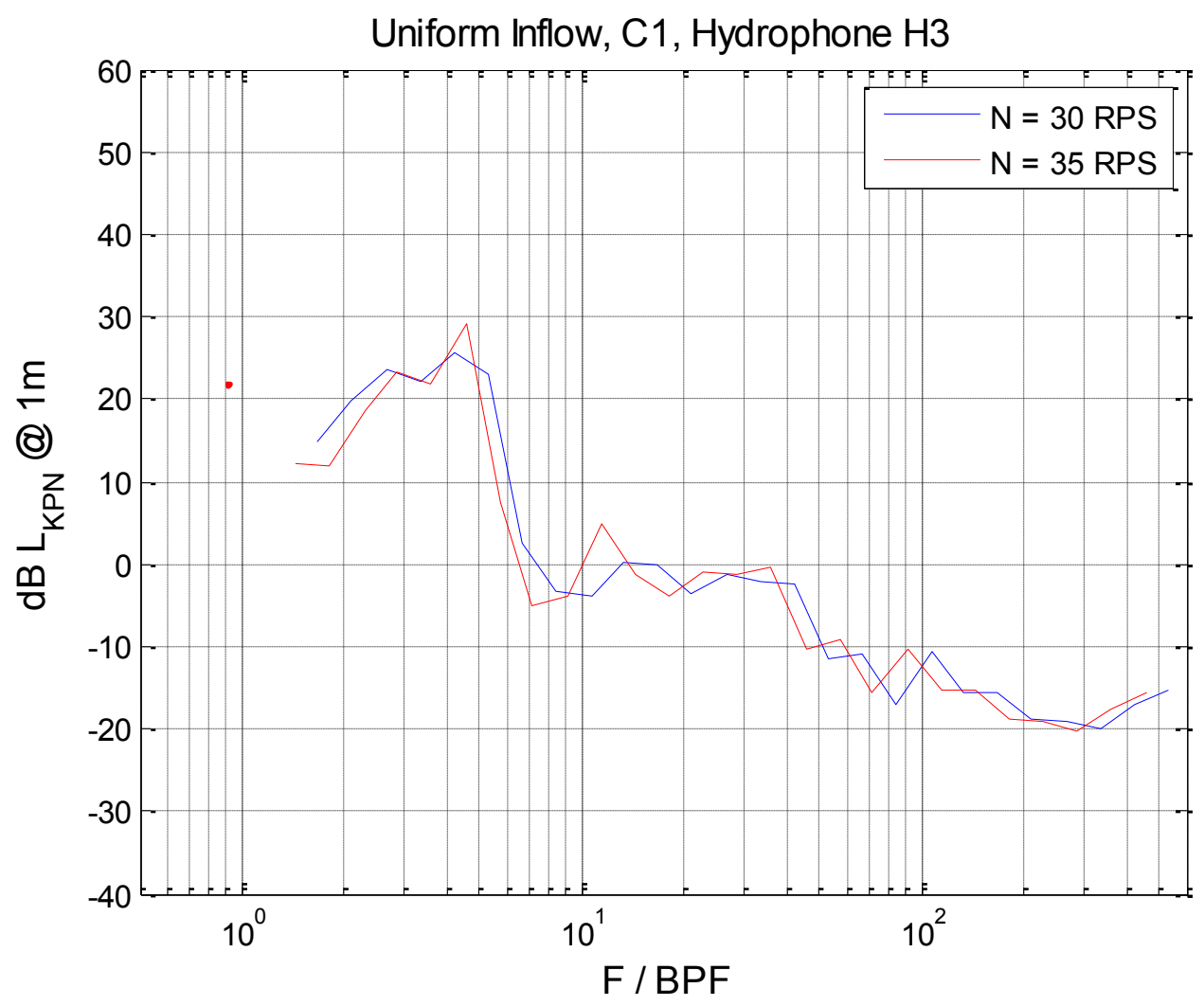

Figure 24: Effect of shaft revolution rate on radiated noise

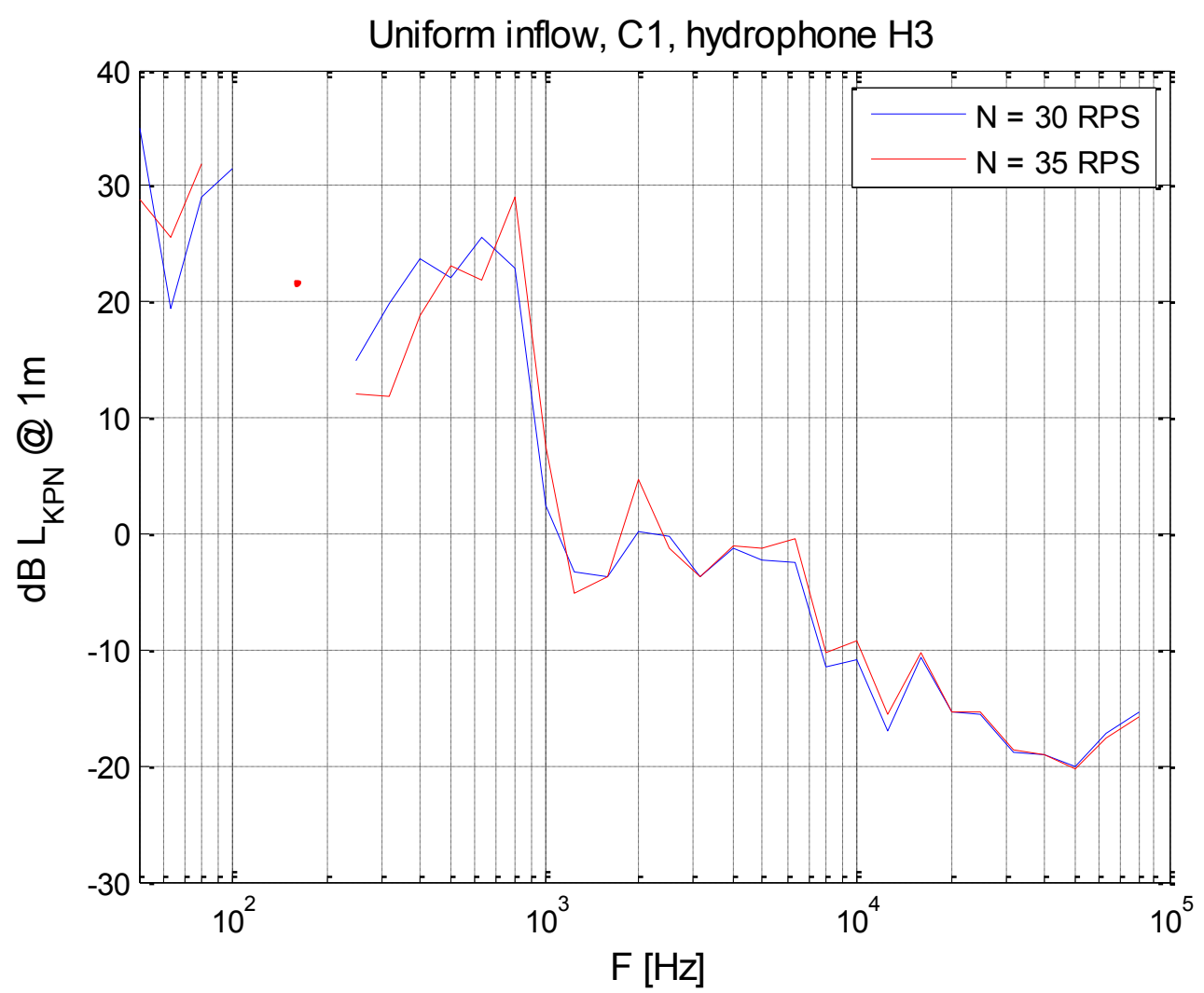

Figure 25: Effect of shaft revolution rate on radiated noise 


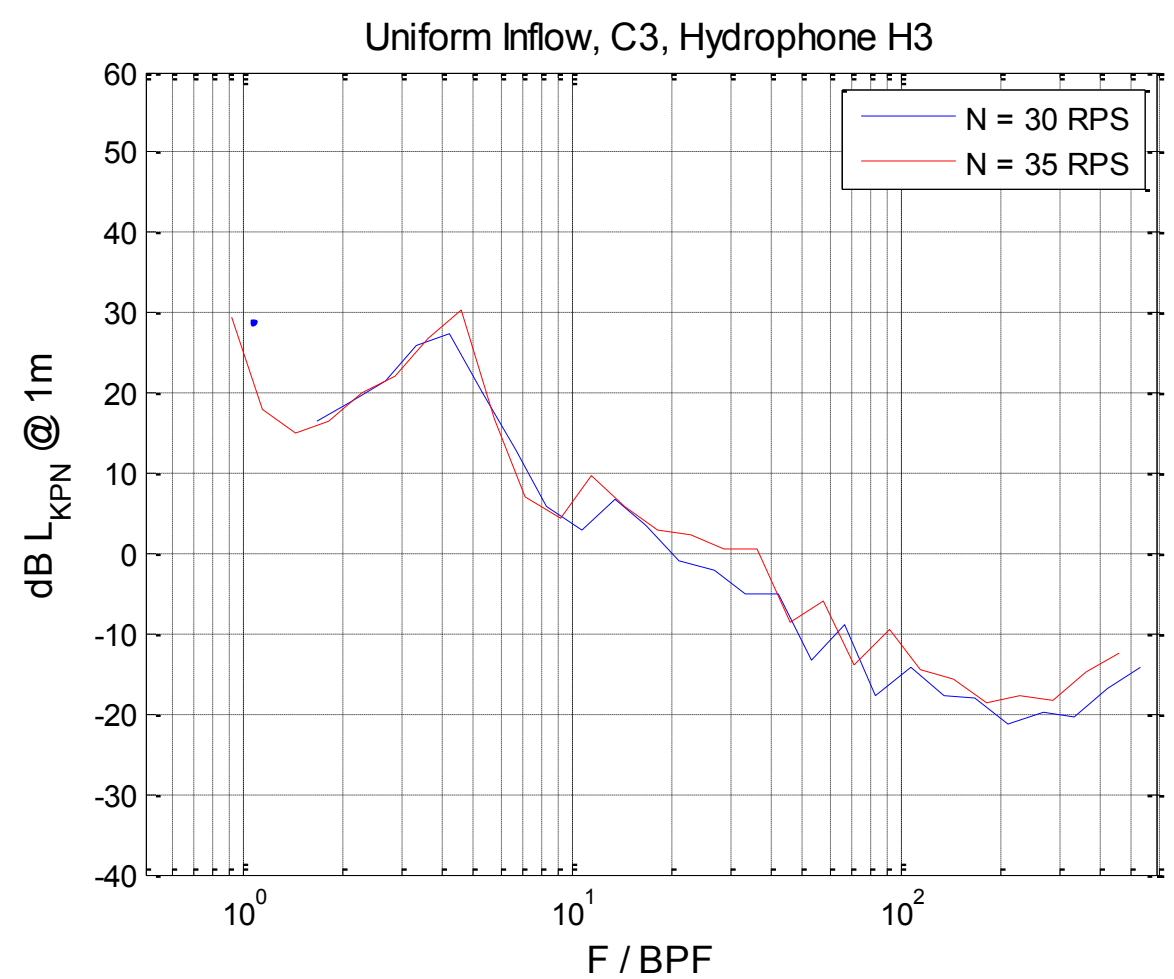

Figure 26: Effect of shaft revolution rate on radiated noise

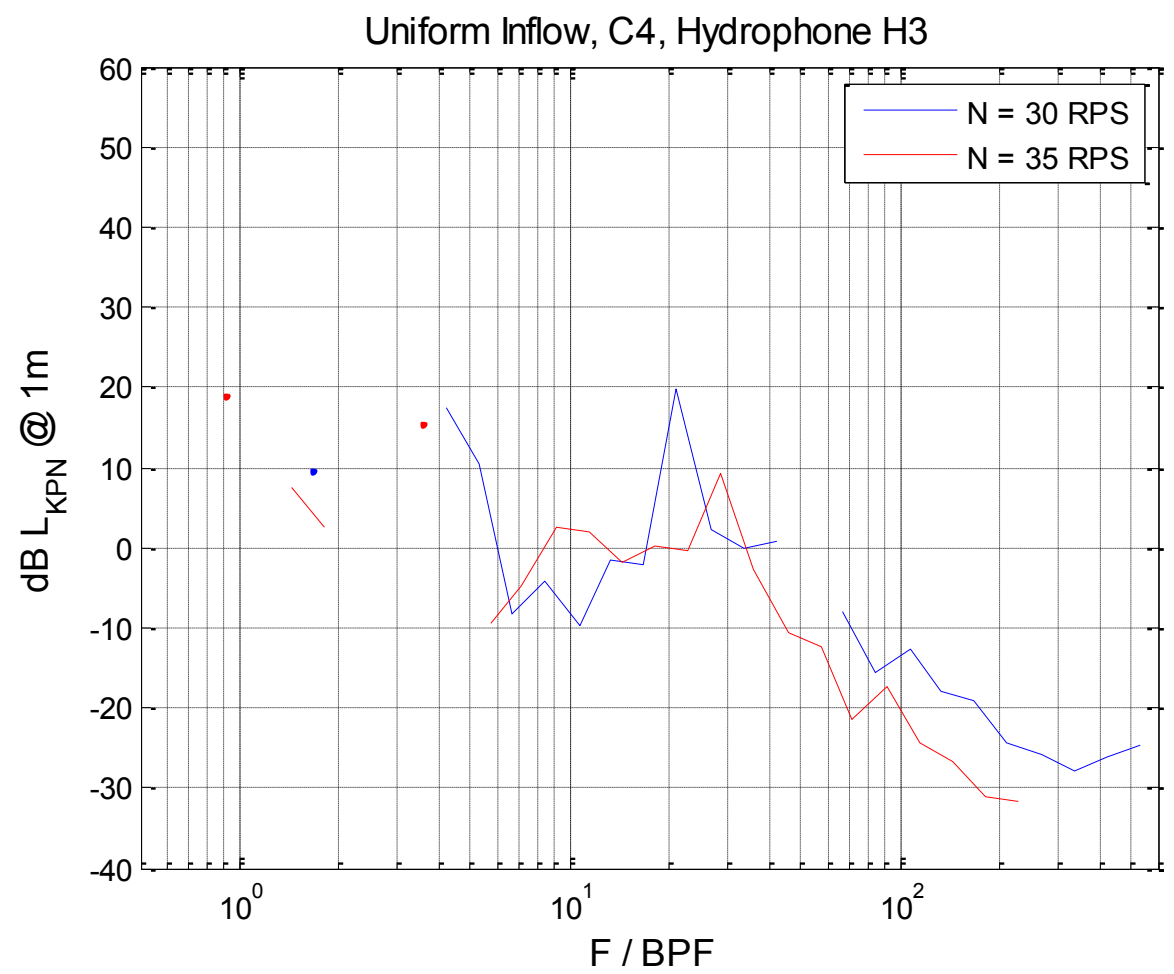

Figure 27: Effect of shaft revolution rate on radiated noise 


\subsection{Sensitivity to thrust coefficient and cavitation number variations}

The last investigation carried out at UNIGE is the sensitivity of the measurements to the variation of parameters defining propeller operating conditions, i.e. thrust coefficient and cavitation number. This analysis is different from that conducted at the ECT on the influence of the advance coefficient because in this case only small variations around the main functioning point are considered. In particular, additional points are defined considering variations of the $10 \%$ of the cavitation number and $5 \%$ of the thrust coefficient.

The analysis of these points allows estimating a range of uncertainty due to those source generally related to the operational conditions, in accordance with the ITTC recommendations (ITTC, 2002).

As usual, reported examples represent the most common situation observed for the different measuring points.

Figure 28 shows results for condition $\mathrm{C}$, already considered as representative of the typical intermediate condition. In this case, spectra measured for the modified functioning conditions present similar characteristics and level variations which are very limited for large part of the considered frequency range. Slightly higher differences are observed at the lower frequencies, in correspondence to the spectral hump associated with the presence of the cavitating tip vortex whose extension and, consequently, noise radiation is, apparently, more sensitive to thrust coefficient and cavitation number. This fact is more evident for some of the intermediate functioning conditions, as visible, for example, for condition C5, shown in Figure 29.

It has to be remarked that small variations observed are in good agreement with cavitation dynamics: the vortex dimension increases with increasing propeller load or decreasing cavitation number, radiated noise behaves accordingly. For example, the spectral hump for conditions with increased cavitation number or decreased thrust coefficient is centred at slightly higher frequencies, in good agreement with the smaller vortex radius; the opposite trend is observed for conditions with higher load or lower cavitation number, which result in increased vortex dimension and, consistently lower frequencies. The same roughly holds for what regards the maximum level of the hump. For condition $\mathrm{C} 1$ levels seem almost unvaried while for condition C5, lower levels are observed decreasing the load or increasing the cavitation number. 
These examples perfectly represent the results observed for conditions C1, C2, C5 and C6 both with uniform inflow and with inclined shaft. In addition, similar results are observed also for the condition with large cavitation extents, C3. Actually, as visible in Figure 30, also spectra measured for this condition present limited variations, except for the tip vortex hump. Practically the only exception to this trend is encountered for the near to inception condition, $\mathrm{C} 4$, as shown in Figure 31. Actually, near to the inception of tip vortex cavitation, larger variations of noise spectra are observed, especially at high frequency where cavitation noise is dominant. Differences between spectra are visible also at lower frequencies; however, these portions of the spectra may be affected by the presence of unwanted noise sources not fully removed by the background correction, as already explained. In this case it can be noticed that lowest levels are observed in correspondence to condition $\mathrm{C} 4$ instead of the $-5 \% \mathrm{~K}_{\mathrm{T}}$ condition. This fact may be a bit surprising since the reduced load should in principle postpone the tip vortex cavitation, however this anomaly may be simply related to the larger variability of results for this conditions.

Summarizing the range of uncertainty related to the hydrodynamic conditions of the propeller is in almost all cases of few $\mathrm{dB}(2-3)$, growing to $5-10 \mathrm{~dB}$ in correspondence to the tip vortex spectral peak. Similar, or even larger, differences are finally observed for the near to inception condition which is, not surprisingly, the most critical measuring condition for all the aspects considered in present work. 


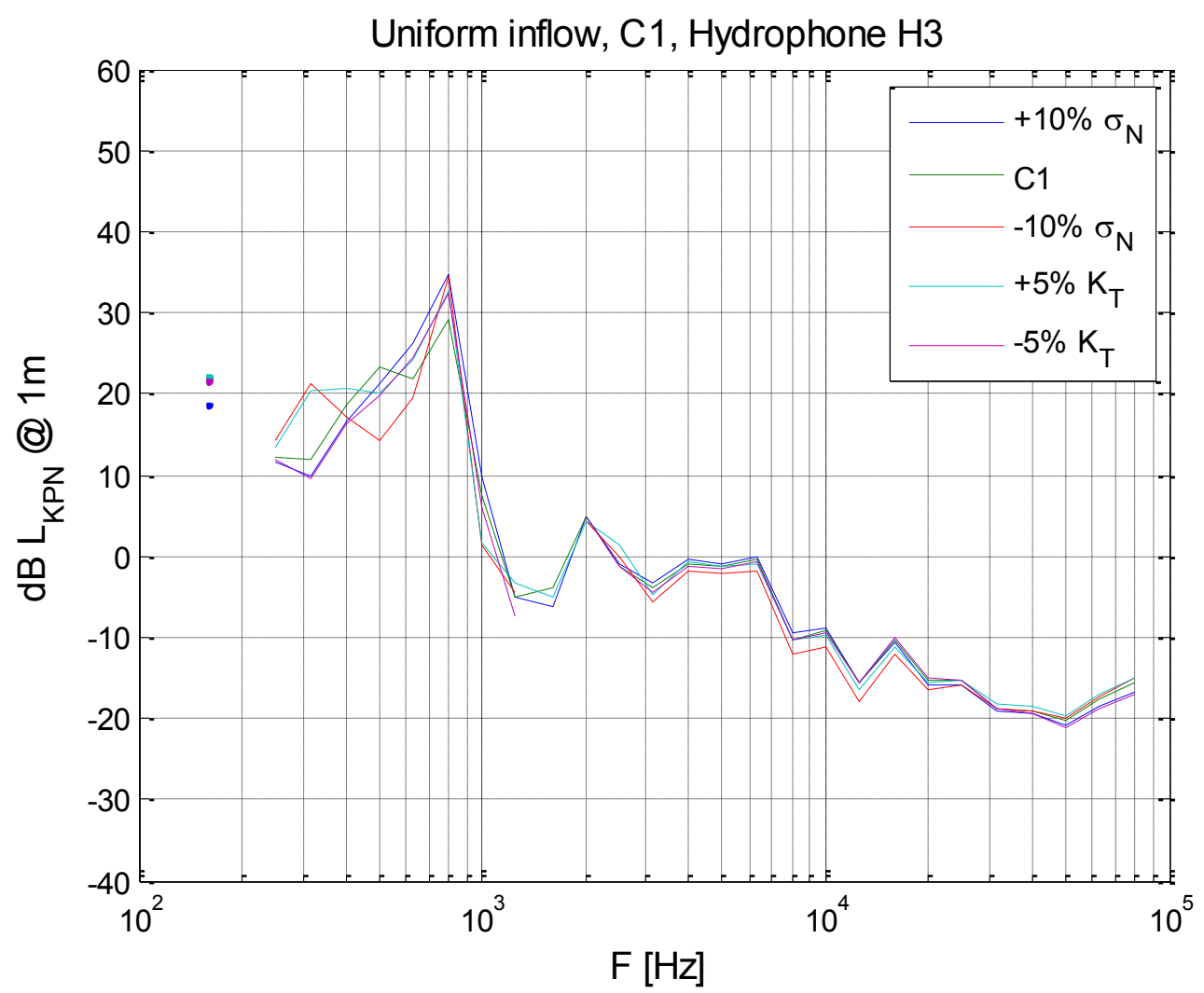

Figure 28: Sensitivity of noise to propeller main functioning parameters

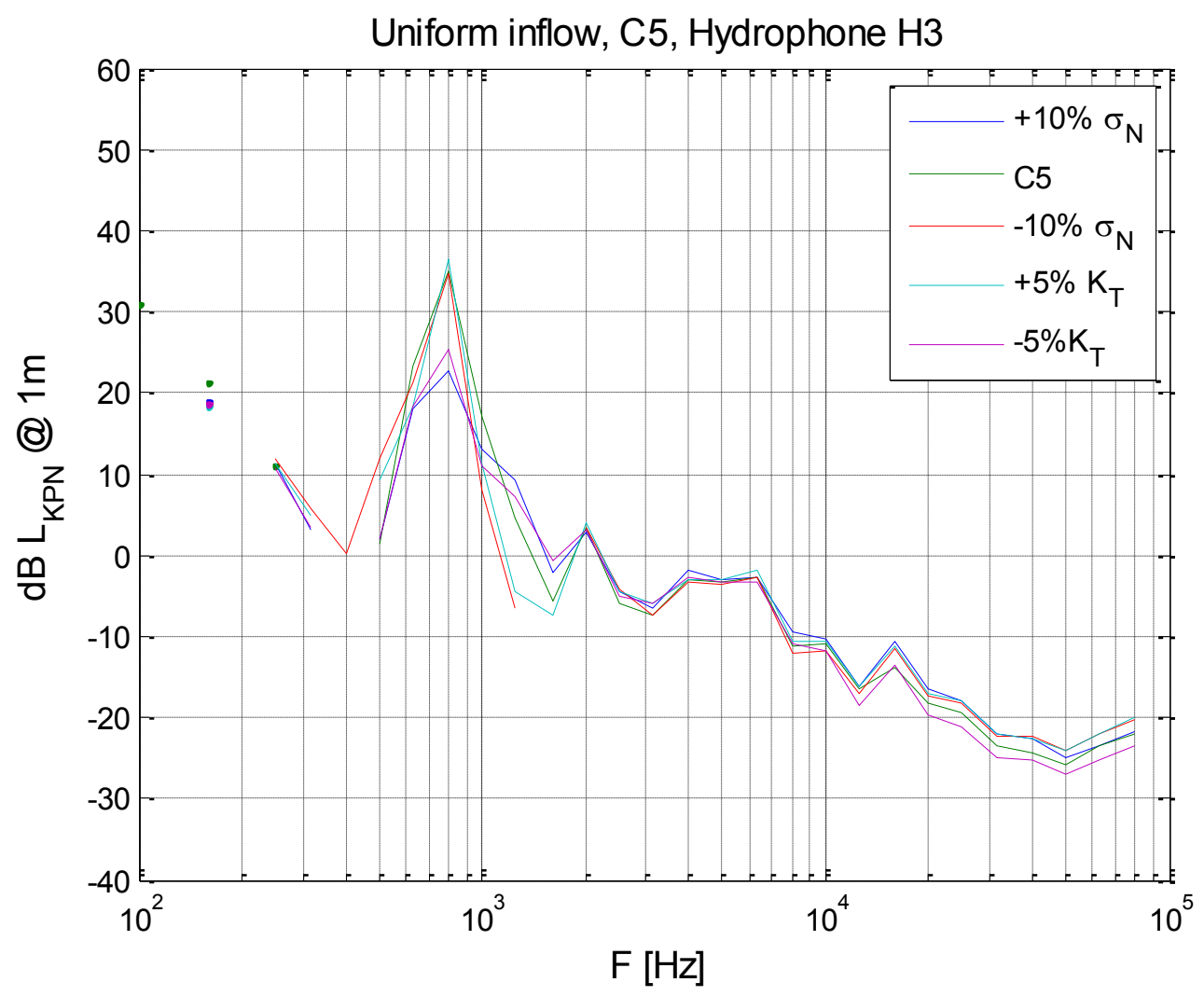

Figure 29: Sensitivity of noise to propeller main functioning parameters 


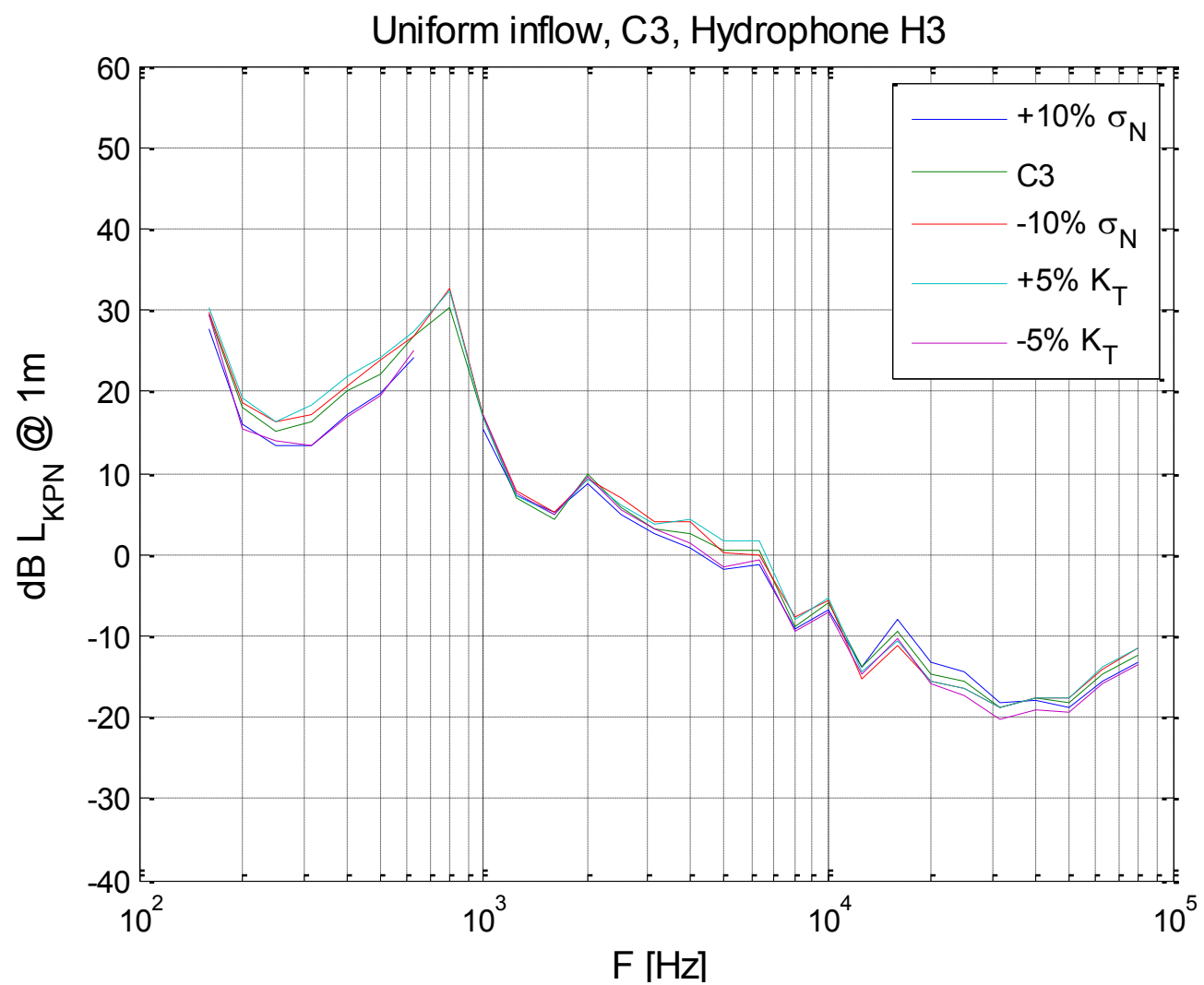

Figure 30: Sensitivity of noise to propeller main functioning parameters

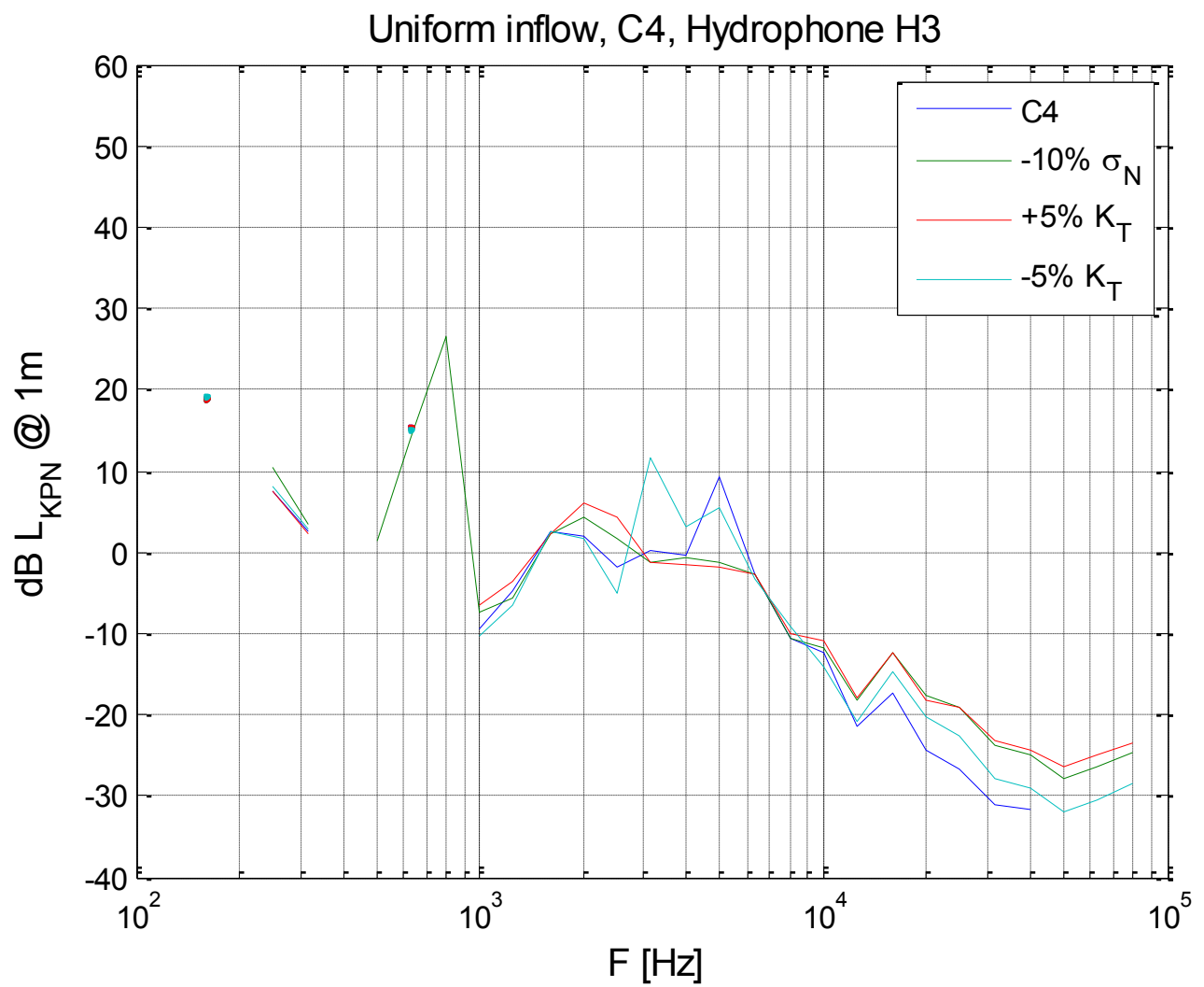

Figure 31: Sensitivity of noise to propeller main functioning parameters 


\subsection{Comparison between ECT and UNIGE noise measurements.}

In this section, the results of the noise tests are directly compared. This, in line with the aims of the Round Robin campaign, allows to analyse the effects of different facilities, equipment and procedures on model scale noise testing.

For what regards UNIGE results, H1 hydrophone is considered for this purpose, instead of the $\mathrm{H} 3$ considered in previous paragraphs. This is because, thanks to its position, hydrophone $\mathrm{H} 1$ is the one likely less affected by near field effects, hence more suitable for the comparisons with other facilities.

To compare the results, it is necessary to assess which is the best way to take into account the differences between the model tests campaigns, as different propeller diameters and different shaft rate. As already discussed in Section 6.3, the adoption of the $\mathrm{K}_{\mathrm{P}}$ coefficient for the sound pressure level and the normalization of the frequency with respect to the blade rate may be sufficient to compare model scale tests.

However, it was decided to compare the extrapolated results in full-scale in order to perform the most general comparison. Considered propeller operational conditions do not correspond to a real ship functioning conditions, as a consequence the extrapolations refer to fictitious conditions characterized by a shaft revolution rate as such to provide the same cavitation number conditions considered in the round robin tests, but with the dimensions of the ship. Practically these fictitious full scale conditions are simply reference conditions for the comparison.

The extrapolations are made applying Equation 11 and Equation 12, in accordance with the ITTC procedures.

$$
\begin{array}{cc}
\frac{\mathrm{f}_{s}}{\mathrm{f}_{m}}=\left(\frac{n_{s}}{n_{m}}\right) & \text { Equation } 11 \\
S P L_{s}=S P L_{m}+20 \log _{10}\left[\frac{r_{m}}{r_{s}} \frac{\rho_{s}}{\rho_{m}}\left(\frac{D_{s}}{D_{m}}\right)^{3}\left(\frac{n_{s}}{n_{m}}\right)^{1.5}\right]\left(\mathrm{dB} \text { re } 1 \mu \mathrm{Pa}^{2} / \mathrm{Hz}\right) & \text { Equation } 12
\end{array}
$$

Where; subscripts "m" and "s" represent model scale and ship scale values of the corresponding parameter respectively. It has to be remarked that, considering similar diameters and same 
reference distance, the application of the noise scaling, from the point of view of comparison, differs from the simple $K_{P}$ normalization only for the exponent applied to the shaft rate, hence comparing $K_{P}$, similar observations could be made.

As far as the measurements are concerned, as it has been discussed in Section 4, the shaft inclination direction seems to have an effect on the propeller inception at ECT, due to the speed profile in the tunnel. As a consequence, the radiated noise may vary depending on this fact, even if in most operational cases the radiated noise are characterized by the developed cavitation patterns, thus differences may become negligible in these cases. In any case, the ECT radiated noise results with inclined shaft are considered for both inclination directions adopted as shown in Figure 32 through 38 for condition C1 through C6, respectively. 


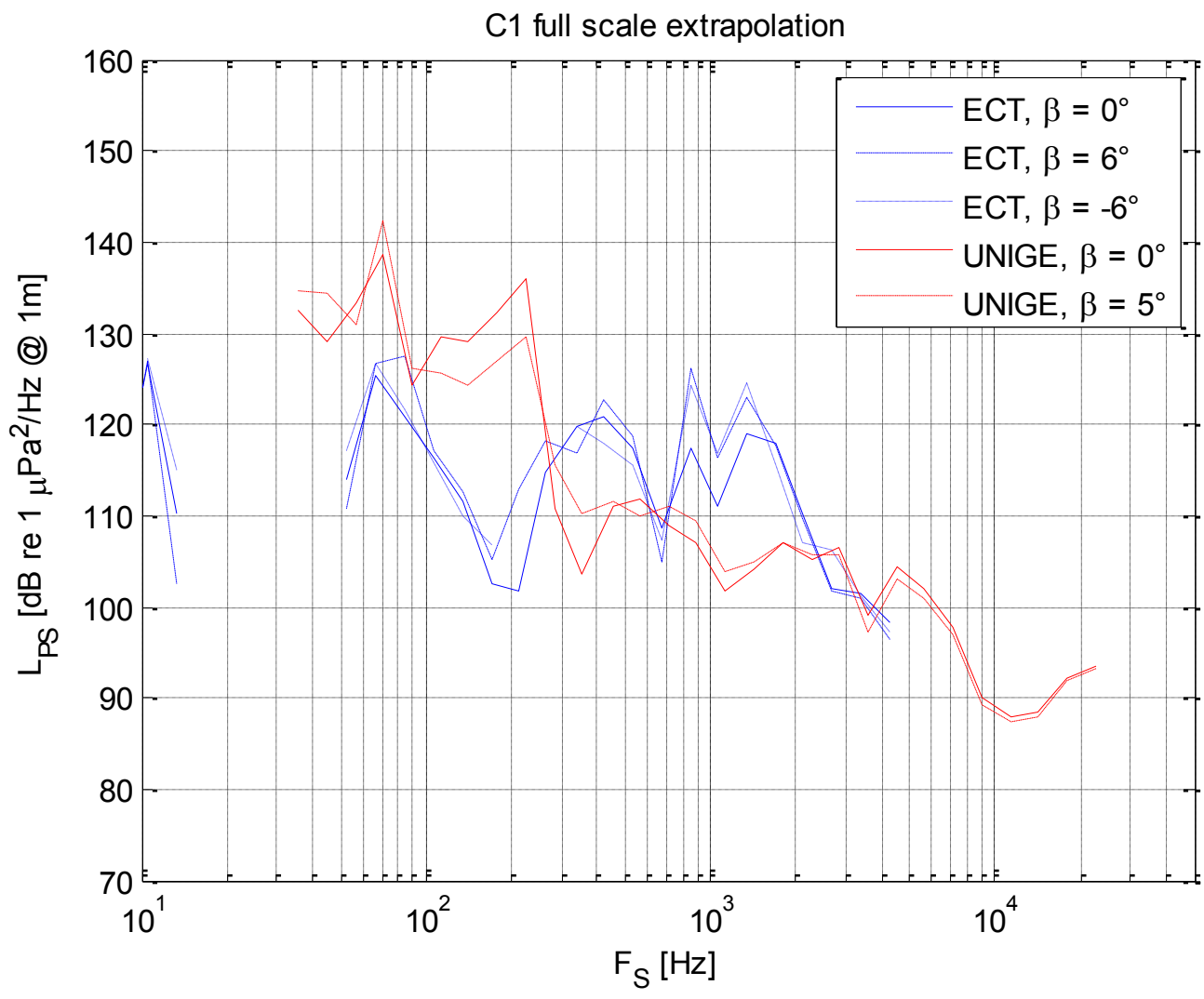

Figure 32: Comparison of noise spectra, condition C1.

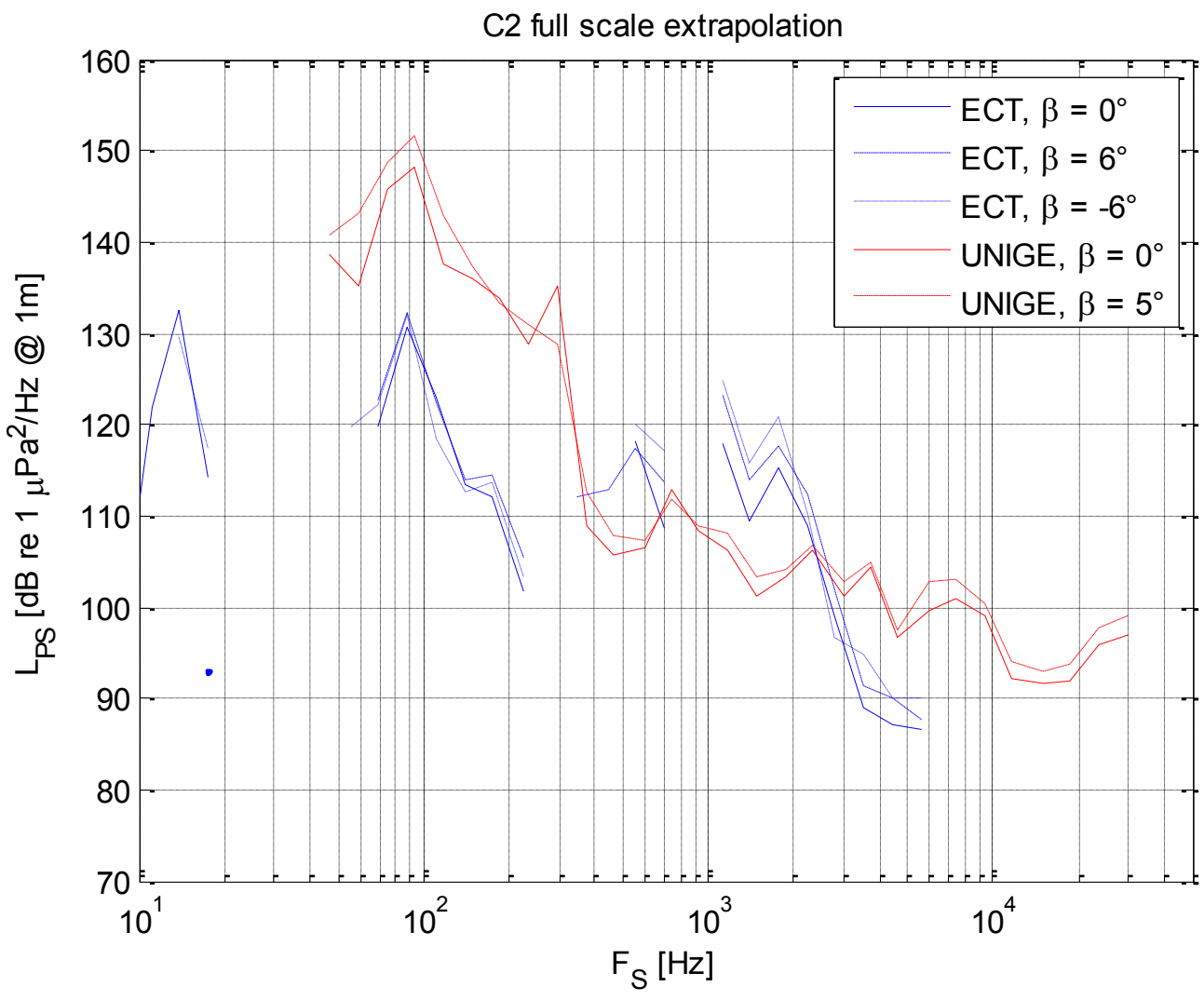

Figure 33: Comparison of noise spectra, condition C2. 


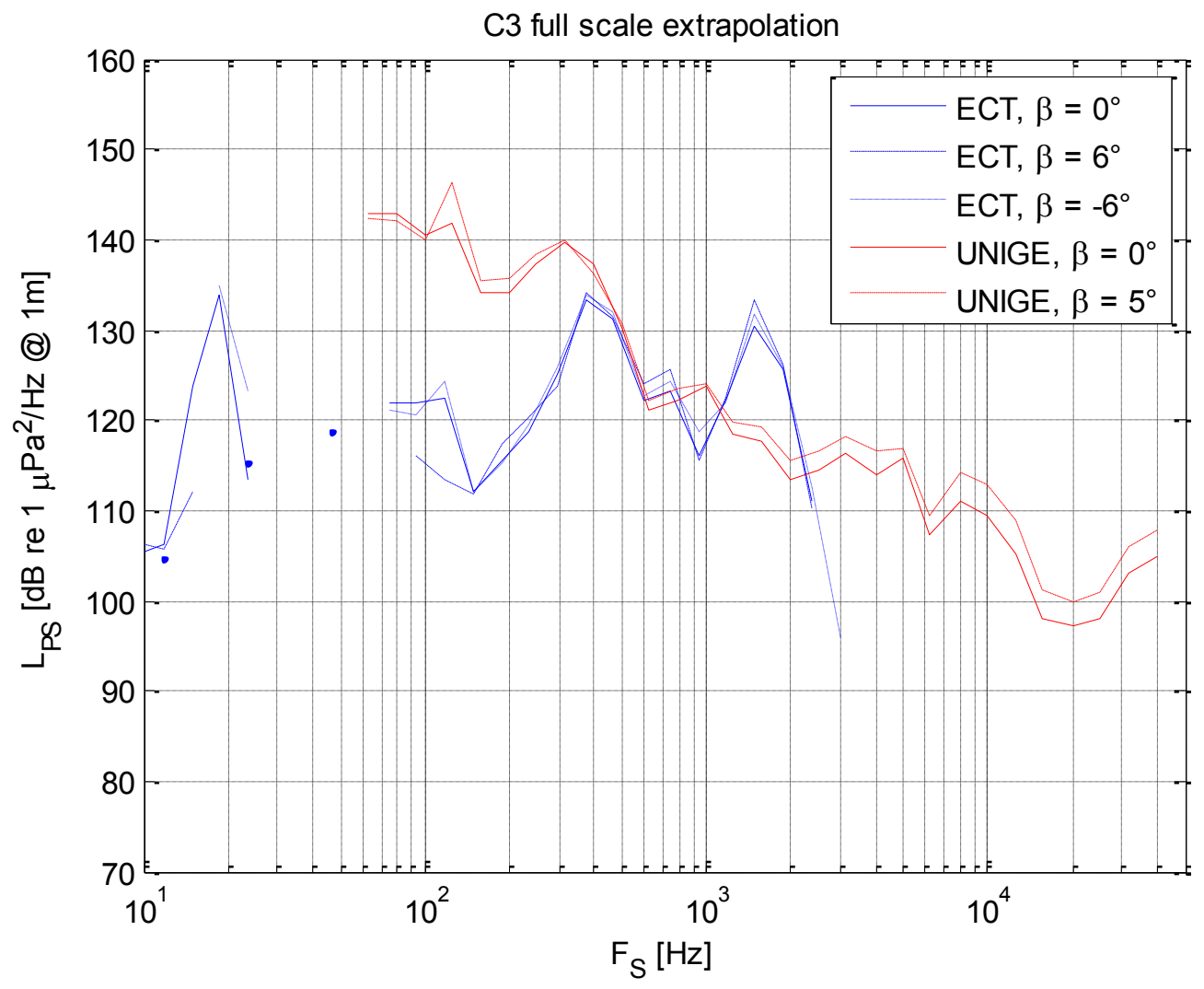

Figure 34: Comparison of noise spectra, condition C3.

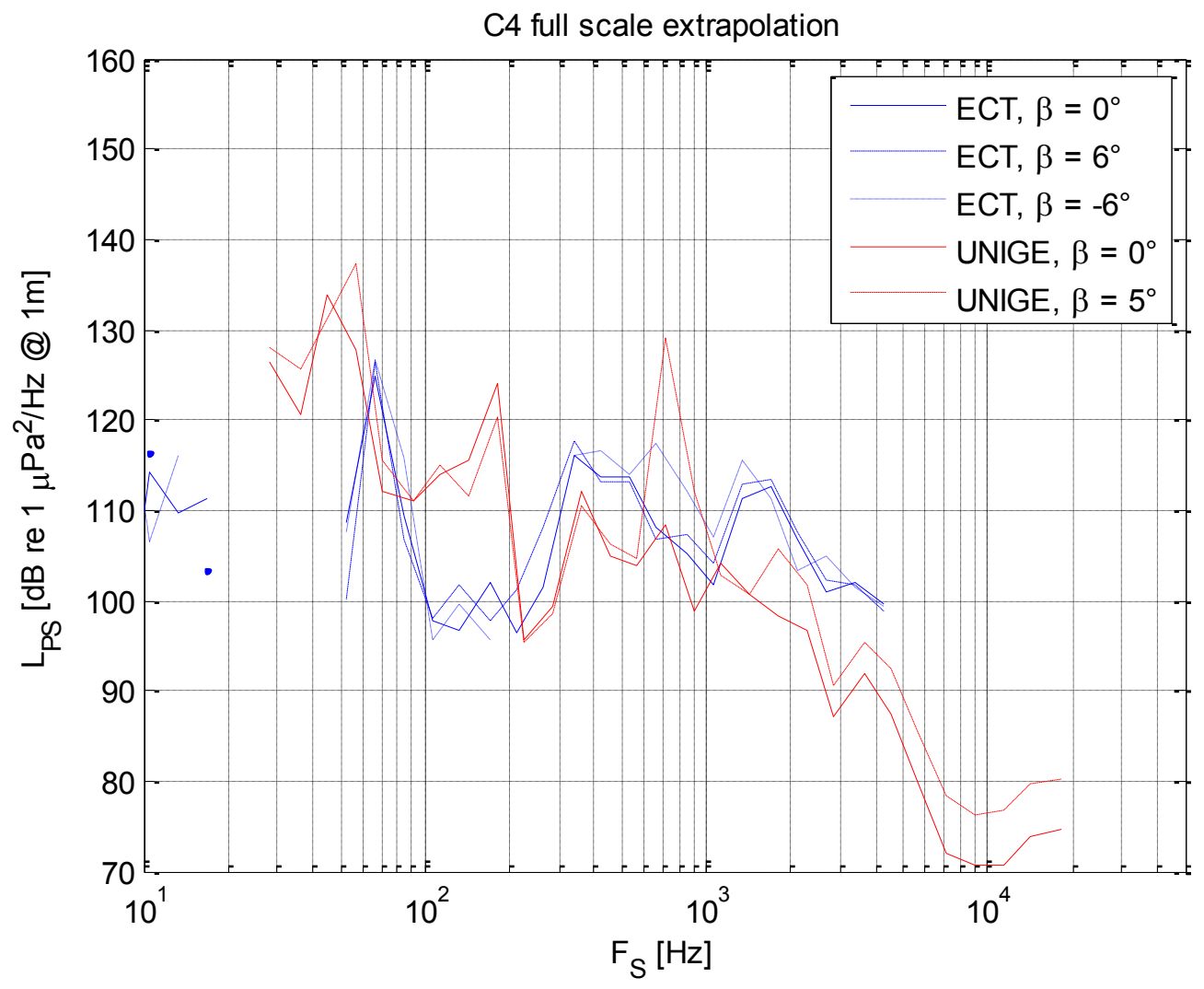

Figure 35: Comparison of noise spectra, condition C4. 


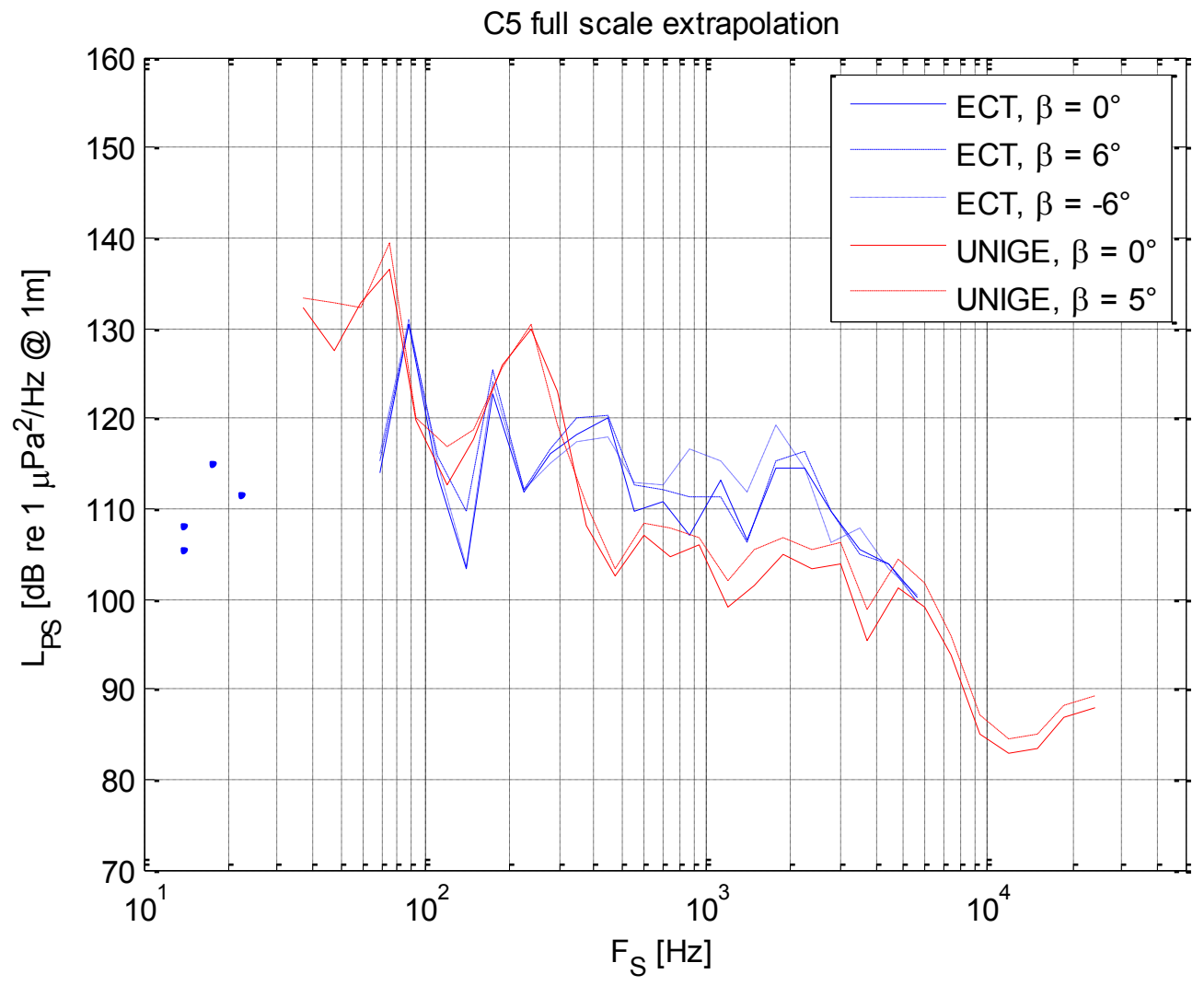

Figure 36: Comparison of noise spectra, condition C5.

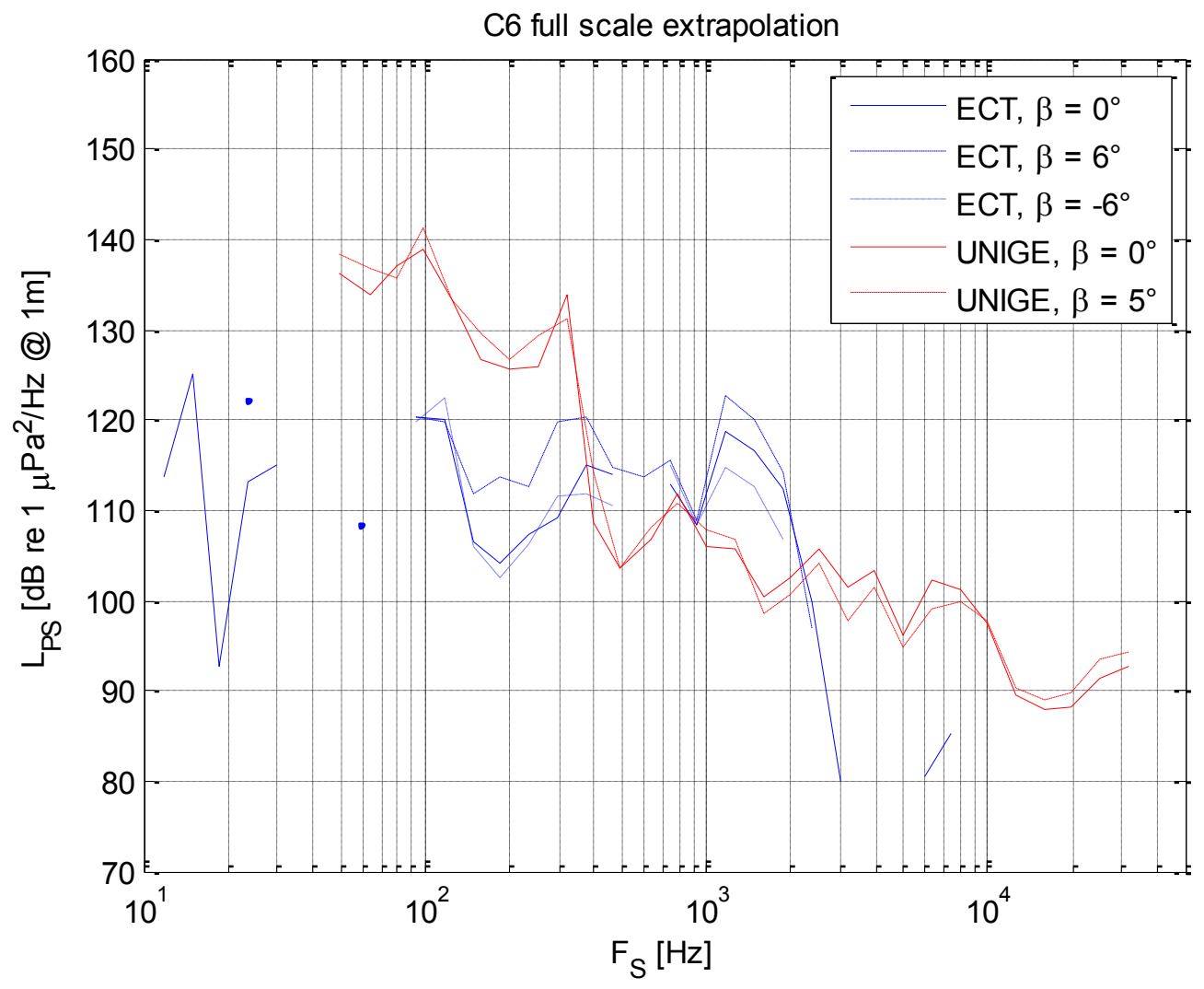

Figure 37: Comparison of noise spectra, condition C6. 
For a better understanding of results, it is important to remark that the initial tests at ECT were carried out in 2013 with pure interest to investigate the implications of shaft inclination on the propeller cavitation noise. A large experimental matrix for a variation of advance coefficient and inclination angles were conducted. For this purpose, the hydrophone was attached on a $30 \mathrm{~mm}$ thick Plexiglas observation window inside a thick walled small steel cylinder and at $0.61 \mathrm{~m}$ distance from the propeller. Moreover, whilst it is preferable to use relatively larger diameter propeller for such investigations, readily available $0.214 \mathrm{~m}$ diameter propeller model was used which was primarily manufactured for the dummy model tests. The implications of aforementioned two aspects resulted in a high signal to noise ratio for the measurements carried out.

By observing the comparative results presented in Figure 32 through 38, one of the first general remarks to be made on the measurements from both facilities is the common trend of the consistently higher sound pressure levels for the inclined shaft conditions in the majority of cases

Regarding the two inclination directions considered at ECT, spectra are rather similar, with some exceptions, as shown in Figure 37 for condition C6. Nevertheless, this aspect seems to have a limited impact on the comparison between noise at the two facilities, contrary to what observed for the cavitation inception.

However, even if for some frequency bands the agreement between the comparative spectra is good, spectral shapes are in general quite different. Keeping in mind the very good agreement between the cavitation extensions, the main reason for this difference is likely related to the propagation effects. Actually, as explained in the above, the first series of the round robin tests were carried out at ECT with a particular hydrophone configuration. This kind of configuration is rather similar to that of the UNIGE hydrophone $\mathrm{H} 1$ as in Figure 4. In section 6.1, it has been shown that the transfer function for that position is rather different from those of the other sensors. As a result, not applying transfer functions, spectra measured by hydrophone $\mathrm{H} 1$ are rather different from those of the two inflow hydrophones, and finally more different from spectra obtained after applying the transfer function correction. A similar phenomenon is likely to occur in the case of the ECT hydrophone placed in the external small cylinder. 
It is already planned in future to repeat measurements at ECT considering different hydrophone positions and transfer functions, in order to further analyse this issue. Nevertheless, further confirmation of the issue by forthcoming test results of the remaining institutions should also shed further light on this in the near future by further reporting of the ongoing round robin tests.

\section{Conclusions}

Cavitation tunnels tests are the most accurate and reliable way for performing full-scale cavitation noise predictions presently. However, a benchmark data for the sole purpose of providing results of a simple case is non-existent for different facilities all around the world to compare with. To address this issue, a round robin test campaign is initiated by the members of Hydro Testing Forum (HTF) Noise Community of Practice. This paper presents the first comparison between the measurements made in the cavitation tunnel of ECT and UNIGE in terms of the propeller performance measurements, cavitation inception points, cavitation observations and most importantly, cavitation induced noise levels. Moreover, a series of investigations are carried out by UNIGE, scrutinizing the effect of hydrophone position, oxygen content, propeller shaft revolution rate, sensitivity to thrust coefficient and cavitation number.

The propeller performance measurements were carried out at fixed inflow velocity whilst varying RPM at ECT whilst UNIGE used the opposite approach by fixing the RPM and varying the inflow velocity. The ECT measurements did not show good agreement against the towing tank results in the high advance coefficient region due to measured torque values being lower. It was apparent that fixing the RPM is a better way of conducting propeller performance tests especially with relatively smaller diameter propeller models. Furthermore, UNIGE also analysed the Reynolds Number effect by repeating performance tests with a range of fixed RPM values.

Cavitation inception comparisons between the two facilities showed intriguing results. While the inception and desinence points were similar for both facilities at the low cavitation number and low thrust coefficient region, the trend of the points diverged as these parameters increased. Various potential reasons such as the nuclei content, inflow velocity profile, inception test procedure differences of the facilities have been discussed in depth. 
Qualitative comparisons of the cavitation extents have shown significant similarity between the two facility observations. Cavitation observations from both ECT and UNIGE have shown to incorporate all of the prominent features of the cavitation and inherent dynamics.

As far as the noise measurements are concerned, the analyses carried out at UNIGE cavitation tunnel provide useful information, especially in the context of an important benchmark, on the sensitivity of measurements on different setups and tunnel functioning parameters.

The first comment may regard hydrophone configurations; in accordance to previous experiences, these tests confirms the large effect that hydrophone configuration may have on radiated noise. This result points out the importance of a reliable transfer function evaluation, furthermore it allows also to better interpret the noise results. Noise propagation likely affects also the preliminary measurements at ECT and this may explain differences between spectra in the two facilities.

From the point of view of the transfer function evaluation, the measurement of the transfer functions for the ECT would be of great interest. Unfortunately, this test cannot be planned at the moment because of long refurbishment activities been carried out due to its relocation. Following its re-commissioning, the determination of the acoustic characterization of the facility is planned by carrying out systematic noise measurements using a known noise source while measuring with hydrophone at various positions within the tunnel. This approach will enable spatial averaging of the measured noise levels hence will enable taking into account for influence of testing environment on the noise transfer function.

For what regards the other analyses, both oxygen content and shaft rate have shown to have a significant influence on noise only for critical conditions, i.e. near to inception or in the presence of bubble absorption problems. Of course these results are influenced by the facility characteristics. As an example, larger tunnels may be less affected by the problem of free bubbles, while facilities with lower background noise should be able to better characterize cavitation inception noise.

Nevertheless, apart the most problematic conditions mentioned, reported results show a limited influence of these parameters on the measured noise spectra, at least in the considered range of variations. From a practical point of view, this means that consistent comparisons can be made also if propeller operational conditions are not reproduced exactly with the same advance speed and propeller rate. Unfortunately, this conclusion is not fully confirmed for the lower advance 
coefficient tested, because of the larger differences present between shaft rate adopted at ECT and UNIGE. However, other institutions involved in the Round Robin campaign may enlarge the range of investigated values, completing this analysis.

Additional functioning points provide a sort of uncertainty band of noise spectra, for what regards propeller hydrodynamic conditions. Analogously with previous considerations, this uncertainty band seems acceptable, with larger variations only for the above mentioned critical conditions.

An additional remark is the possible presence of singing phenomena which is often characterized by poor repeatability and its occurrence is more related to the single propeller model than to the ideal geometry. Thus, in accordance with the aims of the Round Robin tests, they should be always identified and reported when present.

In summary, still within the context of the Round Robin campaign, the first comparison results between the measurements in two different facilities have been reported. The results of the comparative measurements show similar trends and, for certain frequency band the agreement is rather good. However, spectral shapes seem different, probably because of the differences between hydrophone configurations adopted. This result provides further important information on the influence of the setup and facility characteristics on radiated noise tests, which should be carefully taken into account, or at least considered, when comparing experimental results. 


\section{Acknowledgments}

The research is part of the Noise Community of Practice $(\mathrm{CoP})$ activities of the Hydro Testing Forum (HTF) under the task of the round robin test campaign with the model propeller of The Princess Royal.

The Authors acknowledge the members of the HTF Noise CoP for support in publishing this study as well as the permission of the Newcastle University in releasing the blade section details of The Princess Royal. 


\section{Reference}

Abrahamsen, K.A., 2012. The ship as an underwater noise source, in: ECUA 2012 11th European Conference on Underwater Acoustics. Edinburgh, Scotland, 2-6 July, p. 10. doi:10.1121/1.4772953

Aktas, B., Atlar, M., Turkmen, S., Korkut, E., Fitzsimmons, P., 2015. Systematic cavitation tunnel tests of a Propeller in uniform and inclined flow conditions as part of a round robin test campaign. Ocean Engineering 120, 136-151. doi:10.1016/j.oceaneng.2015.12.015

Aktas, B., Atlar, M., Turkmen, S., Shi, W., Sampson, R., Korkut, E., Fitzsimmons, P., 2016. Propeller cavitation noise investigations of a research vessel using medium size cavitation tunnel tests and full-scale trials. Ocean Engineering 120, 122-135. doi:10.1016/j.oceaneng.2015.12.040

Arveson, P.T., Vendittis, D.J., 2000. Radiated noise characteristics of a modern cargo ship. The Journal of the Acoustical Society of America 107, 118-129. doi:10.1121/1.428344

Bark, G., 1985. Prediction of Propeller Cavitation Noise From Model Tests and Its Comparison With Full Scale Data. Transactions of the ASME 107, 112-119. doi:10.1115/1.3242424

Bensow, R.E., Bark, G., 2010a. Simulating Cavitating Flows With Les in Openfoam, in: 5th European Conference on Computational Fluid Dynamics, ECCOMAS CFD. Lisbon, Portugal,14-17 June, p. 18.

Bensow, R.E., Bark, G., 2010b. Implicit LES Predictions of the Cavitating Flow on a Propeller. Journal of Fluids Engineering 132, 41302. doi:10.1115/1.4001342

Bertschneider, H., Bosschers, J., Choi, G.H., Ciappi, E., Farabee, T., Kawakita, C., Tang, D., 2014. Specialist Committee on Hydrodynamic Noise, in: Final Report and Recommendations to the 27th ITTC. Copenhagen, Sweden, p. 45.

Blake, W.K., 1986. Propeller Cavitation Noise, The Problems of Scaling and Prediction. ASME Proceedings of International Symposium on Cavitation and Multiphase Flow Noise.

Blake, W.K., 1984. Aero-hydroacoustics for Ships, Volume I-II, in: David W. Taylor Naval 
Ship Research and Development Center, Naval Sea Systems Command. Washington, DC, USA, p. 627.

Bosschers, J., Lafeber, F.H., Boer, J. de, Bosman, R., Bouvy, A., 2013. Underwater Radiated Noise Measurements With a Silent Towing Carriage in the Depressurized Wave Basin, in: The 3rd International Conference on Advanced Model Measurement Technology for the EU Maritime Industry.

Carlton, J.S., 2007. Ship Resistance and Propulsion, Marine Propellers and Propulsion (Second Edition). doi:10.1016/B978-0-08-097123-0.00012-5

Chahine, G.L., 2004. Nuclei effects on cavitation inception and noise, in: 25th Symposium on Naval Hydrodynamics. St. John's, Newfoundland and Labrador, CANADA, 8-13 August, p. 14.

Costanzo, M., Elefante, M., 1999. Noise Levels Measurements at the INSEAN Circulating Water Channel with a Twin Screw Ship Model, in: CEAS Forum on Aeroaeoustics of Rotors and Propellers. 9-11 June, Rome, Italy.

Hildebrand, J. a., 2009. Anthropogenic and natural sources of ambient noise in the ocean. Marine Ecology Progress Series 395, 5-20. doi:10.3354/meps08353

HTF, 2013. AMT'13, in: International Conference on Advanced Model Measurement Technologies for the Maritime Industry. Gdansk, POLAND.

HTF, 2011. AMT'11, in: International Conference on Advanced Model Measurement Technologies for the Maritime Industry. Newcastle Upon Tyne, UK.

ITTC, 2011. Model - Scale Cavitation Test, in: Recommended Procedures and Guidelines, 7.502-03-03.1. 26th ITTC Specialist Committee on Scaling of Wake Fields, p. 9.

ITTC, 2008. Testing and Extrapolation Methods Propulsion, Propulsor Open Water Test, ITTC - Recommended Procedures and Guidelines, 7.5-02-03-02.1.

ITTC, 2002. Propulsion, Propulsor Uncertainty Analysis, Example for Open Water Test. Recommended Procedures and Guidelines, 7.5-02-03-02.2.

ITTC, 1987. Cavitation committee report, 18th International Towing Tank Conference. Kobe, 
Japan, 18th International Towing Tank Conference, October.

Konno, A., Wakabayashi, K., Yamaguchi, H., Maeda, M., Ishii, N., Soejima, S., Kimura, K., Yamguchi, H., Maeda, M., Ishii, N., Soejima, S., Kimura, K., 2002. On the Mechanism of the Bursting Phenomena of Propeller Tip Vortex Cavitation. Journal of Marine Science and Technology 6, 181-192. doi:10.1007/s007730200006

Korkut, E., Atlar, M., 2002. On the importance of the effect of turbulence in cavitation inception tests of marine propellers. Proceedings of the Royal Society A: Mathematical, Physical and Engineering Sciences 458, 29-48. doi:10.1098/rspa.2001.0852

Korkut, E., Atlar, M., Odabasi, A.Y., 2000. Effect of the Viscous Scale on the Inception of Cavitation and Noise of Marine Propellers 3-5.

Kuiper, G., 2012. Physics of cavitation : Cavitation Inception. Cavitation in ship propulsion $17-27$.

Kuiper, G., 2001. New developments around sheet and tip vortex cavitation on ships' propeller, in: 4th International Symposium on Cavitation. California Institute of Technology, Pasadena, CA, USA, p. 20.

McCormick, B.W., 1962. On Cavitation Produced by a Vortex Trailing from a Lifting Surface. Journal of Basic Engineering 84, 369-378. doi:10.1115/1.3657328

OTT, 2006. Hydrolab DS5X, DS5, and MS5 Water Quality Multiprobes. User Manual.

Pennings, P.C., Bosschers, J., Westerweel, J., van Terwisga, T.J.C., 2015a. Sound signature of propeller tip vortex cavitation. Journal of Fluid Mechanics 778:, 288-313. doi:10.1017/jfm.2015.379

Pennings, P.C., Westerweel, J., van Terwisga, T., Terwisga, T. Van, 2015b. Sound signature of propeller tip vortex cavitation. Journal of Physics: Conference Series 656:, 5. doi:10.1088/1742-6596/656/1/012186

Renilson, 2009. Reducing underwater noise pollution from large commercial vessels, Renilson Marine Consulting Pty Ltd,The International Fund for Animal Welfare.

Richardson, W.J.W., Greene, C.R., Jr., Malme, C.I.C., Thomson, D.H.D., Jr, C.G., Malme, 
C.I.C., Thomson, D.H.D., 2013. Marine mammals and noise. Elsevier Science.

Ross, D., 1976. Mechanics of Underwater Noise. Peninsula Publishing, California, USA. doi:10.1016/B978-0-08-021182-4.50014-3

SONIC, 2012. Suppression Of underwater Noise Induced by Cavitation, in: European Union Framework Programme 7. FP7-SST-2012-RTD-1- SST.2012.1.1-1. - Assessment and mitigation of noise impacts of the maritime transport on the marine environment (coordinated topic within the framework of the "Ocean of Tomorrow"), FP7, Grant agreement no: 314394 .

Tani, G., Viviani, M., Armelloni, E., Nataletti, M., 2015a. Cavitation tunnel acoustic characterisation and application to model propeller radiated noise measurements at different functioning conditions. Proceedings of the Institution of Mechanical Engineers, Part M: Journal of Engineering for the Maritime Environment 17. doi:10.1177/1475090214563860

Tani, G., Viviani, M., Gaggero, T., Hallander, J., Johansson, T., 2015b. Evaluation Of Methods To Measure Acoustic Transer Functions In Cavitation Tunnels, in: The 4th International Conference on Advanced Model Measurement Technologies for the Maritime Industry, AMT'15. Istanbul, Turkey.

Van der Graaf, A.J., Ainslie, M.A., André, M., Brensing, K., Dalen, J., Dekeling, R.P.A., Robinson, S., Tasker, M.L., Thomsen, F., Werner, S., 2012. European Marine Strategy Framework Directive- Good Environmental Status (MSFD GES).

White, P., Pace, F., 2010. The Impact of Underwater Ship Noise on Marine Mammals. 1st IMarEST Ship Noise and Vibration Conference.

YSI Environmental, 2006. YSI 550A Dissolved Oxygen Instrument. Operations Manual. 
This appendix provides the offset table for the propeller blade sections provided by Table 6 . Details of the propeller geometry will enable this benchmark test case to be not only replicated in experimental facilities but also to provide a validation case for numerical simulations.

Table 7 Offset table for model propeller blade sections of The Princess Royal propeller

\begin{tabular}{|c|c|c|c|c|}
\hline & $\begin{array}{c}\mathbf{r} / \mathbf{R}=\mathbf{0 . 2 0} \\
\% \mathrm{C} \text { from }\end{array}$ \\
\hline LE & $\mathrm{x} \mathrm{mm}$ & Back Ord & Thickness & Face Ord \\
\hline 0 & 0.00 & 2.82 & 5.64 & -2.82 \\
\hline 2.5 & 6.14 & 5.29 & 9.79 & -4.50 \\
\hline 5 & 12.28 & 7.47 & 13.41 & -5.94 \\
\hline 10 & 24.56 & 10.78 & 18.69 & -7.91 \\
\hline 20 & 49.12 & 15.27 & 25.61 & -10.35 \\
\hline 30 & 73.68 & 17.91 & 29.68 & -11.76 \\
\hline 40 & 98.24 & 19.13 & 31.69 & -12.57 \\
\hline 50 & 122.81 & 19.07 & 31.76 & -12.69 \\
\hline 60 & 147.37 & 17.81 & 29.78 & -11.97 \\
\hline 70 & 171.93 & 15.37 & 25.82 & -10.45 \\
\hline 80 & 196.49 & 11.77 & 19.90 & -8.13 \\
\hline 90 & 221.05 & 7.01 & 12.01 & -5.00 \\
\hline 95 & 233.33 & 4.18 & 7.32 & -3.13 \\
\hline 100 & 245.61 & 1.25 & 2.50 & -1.25 \\
\hline \multicolumn{5}{|c|}{$\mathbf{r} / \mathbf{R}=\mathbf{0 . 2 5}$} \\
\hline $\mathrm{LE}$ & $\mathrm{x} \mathrm{mm}$ & Back Ord & Thickness & Face Ord \\
\hline 0 & 0.00 & 2.58 & 5.15 & -2.58 \\
\hline 2.5 & 6.58 & 5.12 & 9.24 & -4.12 \\
\hline 5 & 13.15 & 7.29 & 12.65 & -5.35 \\
\hline 10 & 26.30 & 10.62 & 17.63 & -7.00 \\
\hline 20 & 52.61 & 15.18 & 24.16 & -8.97 \\
\hline 30 & 78.91 & 17.88 & 27.99 & -10.11 \\
\hline 40 & 105.21 & 19.09 & 29.89 & -10.80 \\
\hline 50 & 131.52 & 19.00 & 29.95 & -10.95 \\
\hline 60 & 157.82 & 17.72 & 28.09 & -10.36 \\
\hline 70 & 184.12 & 15.28 & 24.36 & -9.07 \\
\hline 80 & 210.42 & 11.69 & 18.77 & -7.09 \\
\hline 90 & 236.73 & 6.93 & 11.33 & -4.40 \\
\hline 95 & 249.88 & 4.16 & 7.00 & -2.84 \\
\hline 100 & 263.03 & 1.25 & 2.50 & -1.25 \\
\hline
\end{tabular}




$\begin{array}{ccccc}\begin{array}{c}\mathbf{r} \mathbf{R}=\mathbf{0 . 3 0} \\ \text { \%C from }\end{array} & & & & \\ \text { LE } & \text { x mm } & \text { Back Ord } & \text { Thickness } & \text { Face Ord } \\ 0 & 0.00 & 2.50 & 5.00 & -2.50 \\ 2.5 & 6.96 & 4.91 & 8.68 & -3.76 \\ 5 & 13.93 & 7.05 & 11.88 & -4.83 \\ 10 & 27.85 & 10.35 & 16.56 & -6.20 \\ 20 & 55.70 & 14.90 & 22.69 & -7.79 \\ 30 & 83.55 & 17.59 & 26.29 & -8.70 \\ 40 & 11.40 & 18.78 & 28.08 & -9.30 \\ 50 & 139.26 & 18.68 & 28.13 & -9.46 \\ 60 & 167.11 & 17.41 & 26.38 & -8.98 \\ 70 & 194.96 & 14.99 & 22.88 & -7.88 \\ 80 & 222.81 & 11.45 & 17.63 & -6.18 \\ 90 & 250.66 & 6.77 & 10.64 & -3.87 \\ 95 & 264.58 & 4.11 & 6.70 & -2.59 \\ 100 & 278.51 & 1.25 & 2.50 & -1.25 \\ & & & & \\ \mathbf{r} / \mathbf{R}=\mathbf{0 . 4 0} & & & & \\ \% \mathrm{C} \text { from } & & & & \\ \text { LE } & \text { x mm } & \text { Back Ord } & \text { Thickness } & \text { Face Ord } \\ 0 & 0.00 & 2.17 & 4.34 & -2.17 \\ 2.5 & 7.72 & 4.35 & 7.56 & -3.21 \\ 5 & 15.44 & 6.28 & 10.35 & -4.07 \\ 10 & 30.87 & 9.28 & 14.42 & -5.15 \\ 20 & 61.74 & 13.43 & 19.77 & -6.34 \\ 30 & 92.62 & 15.88 & 22.91 & -7.02 \\ 40 & 123.49 & 16.96 & 24.46 & -7.51 \\ 50 & 154.36 & 16.85 & 24.51 & -7.66 \\ 60 & 185.23 & 15.69 & 22.99 & -7.29 \\ 70 & 216.10 & 13.51 & 19.93 & -6.42 \\ 80 & 246.98 & 10.31 & 15.36 & -5.06 \\ 90 & 277.85 & 6.08 & 9.27 & -3.19 \\ 95 & 293.28 & 3.67 & 5.84 & -2.17 \\ 100 & 308.72 & 1.25 & 2.50 & -1.25\end{array}$




$\begin{array}{ccccc}\begin{array}{c}\mathbf{r} \mathbf{R}=\mathbf{0 . 5 0} \\ \text { \%C from }\end{array} & & & & \\ \text { LE } & \text { x mm } & \text { Back Ord } & \text { Thickness } & \text { Face Ord } \\ 0 & 0.00 & 1.85 & 3.70 & -1.85 \\ 2.5 & 8.32 & 3.75 & 6.44 & -2.70 \\ 5 & 16.64 & 5.43 & 8.82 & -3.39 \\ 10 & 33.28 & 8.05 & 12.29 & -4.25 \\ 20 & 66.57 & 11.68 & 16.85 & -5.17 \\ 30 & 99.85 & 13.83 & 19.52 & -5.69 \\ 40 & 133.13 & 14.77 & 20.85 & -6.08 \\ 50 & 166.42 & 14.67 & 20.89 & -6.22 \\ 60 & 199.70 & 13.65 & 19.59 & -5.94 \\ 70 & 232.98 & 11.75 & 16.99 & -5.24 \\ 80 & 266.26 & 8.96 & 13.09 & -4.13 \\ 90 & 299.55 & 5.45 & 8.24 & -2.79 \\ 95 & 316.19 & 3.45 & 5.52 & -2.07 \\ 100 & 332.83 & 1.25 & 2.50 & -1.25 \\ & & & & \\ \mathbf{r} / \mathbf{R}=\mathbf{0 . 6 0} & & & & \\ \% \text { C from } & & & & \\ \text { LE } & \text { x mm } & \text { Back Ord } & \text { Thickness } & \text { Face Ord } \\ 0 & 0.00 & 1.50 & 3.00 & -1.50 \\ 2.5 & 8.72 & 3.14 & 5.32 & -2.19 \\ 5 & 17.43 & 4.57 & 7.29 & -2.72 \\ 10 & 34.86 & 6.80 & 10.16 & -3.36 \\ 20 & 69.72 & 9.92 & 13.93 & -4.01 \\ 30 & 104.58 & 11.76 & 16.14 & -4.38 \\ 40 & 139.44 & 12.55 & 17.23 & -4.68 \\ 50 & 174.31 & 12.46 & 17.27 & -4.81 \\ 60 & 209.17 & 11.59 & 16.19 & -4.60 \\ 70 & 244.03 & 9.97 & 14.04 & -4.07 \\ 80 & 278.89 & 7.60 & 10.82 & -3.22 \\ 90 & 313.75 & 4.65 & 6.90 & -2.25 \\ 95 & 331.18 & 3.00 & 4.75 & -1.75 \\ 100 & 348.61 & 1.25 & 2.50 & -1.25\end{array}$




$\begin{array}{ccccc}\begin{array}{c}\mathbf{r} \mathbf{R}=\mathbf{0 . 7 0} \\ \text { \%C from }\end{array} & & & & \\ \text { LE } & \text { x mm } & \text { Back Ord } & \text { Thickness } & \text { Face Ord } \\ 0 & 0.00 & 1.25 & 2.50 & -1.25 \\ 2.5 & 8.81 & 2.53 & 4.21 & -1.68 \\ 5 & 17.62 & 3.71 & 5.76 & -2.05 \\ 10 & 35.24 & 5.56 & 8.03 & -2.47 \\ 20 & 70.49 & 8.15 & 11.01 & -2.85 \\ 30 & 105.73 & 9.69 & 12.75 & -3.06 \\ 40 & 140.98 & 10.34 & 13.62 & -3.28 \\ 50 & 176.22 & 10.26 & 13.65 & -3.39 \\ 60 & 211.46 & 9.54 & 12.80 & -3.26 \\ 70 & 246.71 & 8.20 & 11.10 & -2.90 \\ 80 & 281.95 & 6.24 & 8.55 & -2.31 \\ 90 & 317.20 & 3.85 & 5.55 & -1.70 \\ 95 & 334.82 & 2.56 & 4.00 & -1.44 \\ 100 & 352.44 & 1.25 & 2.50 & -1.25 \\ & & & & \\ \mathbf{r} / \mathbf{R}=\mathbf{0 . 8 0} & & & & \\ \% \mathrm{C} \text { from } & & & & \\ \text { LE } & \text { x mm } & \text { Back Ord } & \text { Thickness } & \text { Face Ord } \\ 0 & 0.00 & 1.25 & 2.50 & -1.25 \\ 2.5 & 8.39 & 2.11 & 3.48 & -1.37 \\ 5 & 16.77 & 2.84 & 4.23 & -1.39 \\ 10 & 33.55 & 4.30 & 5.90 & -1.60 \\ 20 & 67.10 & 6.35 & 8.08 & -1.73 \\ 30 & 100.64 & 7.57 & 9.37 & -1.80 \\ 40 & 134.19 & 8.08 & 10.00 & -1.92 \\ 50 & 167.74 & 8.00 & 10.02 & -2.02 \\ 60 & 201.29 & 7.44 & 9.40 & -1.96 \\ 70 & 234.84 & 6.38 & 8.15 & -1.77 \\ 80 & 268.38 & 4.96 & 6.50 & -1.54 \\ 90 & 301.93 & 3.23 & 4.57 & -1.34 \\ 95 & 318.71 & 2.27 & 3.55 & -1.28 \\ 100 & 335.48 & 1.25 & 2.50 & -1.25\end{array}$




$\begin{array}{ccccc}\mathbf{r} / \mathbf{R}=\mathbf{0 . 9 0} & & & & \\ \text { \%C from } & & & & \\ \text { LE } & \text { x mm } & \text { Back Ord } & \text { Thickness } & \text { Face Ord } \\ 0 & 0.00 & 1.25 & 2.50 & -1.25 \\ 2.5 & 7.01 & 1.74 & 2.90 & -1.16 \\ 5 & 14.01 & 2.19 & 3.25 & -1.06 \\ 10 & 28.02 & 3.03 & 3.97 & -0.94 \\ 20 & 56.04 & 4.38 & 5.16 & -0.79 \\ 30 & 84.06 & 5.24 & 5.98 & -0.75 \\ 40 & 112.08 & 5.59 & 6.39 & -0.80 \\ 50 & 140.11 & 5.53 & 6.40 & -0.87 \\ 60 & 168.13 & 5.13 & 6.00 & -0.87 \\ 70 & 196.15 & 4.46 & 5.32 & -0.86 \\ 80 & 224.17 & 3.57 & 4.49 & -0.92 \\ 90 & 252.19 & 2.48 & 3.50 & -1.02 \\ 95 & 266.20 & 1.88 & 3.00 & -1.12 \\ 100 & 280.21 & 1.25 & 2.50 & -1.25 \\ & & & & \\ \mathbf{r} / \mathbf{R}=\mathbf{0 . 9 5} & & & & \\ \% \mathrm{C} \text { from } & & & & \\ \text { LE } & \text { x mm } & \text { Back Ord } & \text { Thickness } & \text { Face Ord } \\ 0 & 0.00 & 1.25 & 2.50 & -1.25 \\ 2.5 & 5.41 & 1.56 & 2.69 & -1.13 \\ 5 & 10.82 & 1.85 & 2.85 & -1.00 \\ 10 & 21.64 & 2.38 & 3.20 & -0.82 \\ 20 & 43.28 & 3.25 & 3.81 & -0.56 \\ 30 & 64.93 & 3.83 & 4.29 & -0.47 \\ 40 & 86.57 & 4.09 & 4.59 & -0.50 \\ 50 & 108.21 & 4.04 & 4.59 & -0.55 \\ 60 & 129.85 & 3.81 & 4.43 & -0.62 \\ 70 & 151.49 & 3.40 & 4.10 & -0.70 \\ 80 & 173.14 & 2.82 & 3.65 & -0.83 \\ 90 & 194.78 & 2.10 & 3.11 & -1.01 \\ 95 & 205.60 & 1.69 & 2.80 & -1.11 \\ 100 & 216.42 & 1.25 & 2.50 & -1.25\end{array}$

